\title{
Modeling the Subsurface Structure of Sunspots
}

\author{
H. Moradi - C. Baldner • A.C. Birch • D.C. Braun • R.H. Cameron • T.L. Duvall Jr. • \\ L. Gizon · D. Haber · S.M. Hanasoge · B.W. Hindman · J. Jackiewicz • E. Khomenko • \\ R. Komm • P. Rajaguru • M. Rempel • M. Roth • R. Schlichenmaier • H. Schunker • \\ H.C. Spruit • K.G. Strassmeier • M.J. Thompson • S. Zharkov
}

Received: 27 December 2009 / Accepted: 23 August 2010 / Published online: 19 October 2010

(C) Springer Science+Business Media B.V. 2010

Invited Review.

H. Moradi · R.H. Cameron · L. Gizon $(\varangle) \cdot$ S.M. Hanasoge $\cdot$ H. Schunker Max-Planck-Institut für Sonnensystemforschung, 37191 Katlenburg-Lindau, Germany e-mail: gizon@mps.mpg.de

C. Baldner

Department of Astronomy, Yale University, P.O. Box 208101, New Haven, CT 06520, USA

A.C. Birch · D.C. Braun

A Division of NorthWest Research Associates, Inc., Colorado Research Associates, 3380 Mitchell Lane, Boulder, CO 80301-5410, USA

T.L. Duvall Jr.

Laboratory for Solar Physics, NASA/Goddard Space Flight Center, Greenbelt, MD 20771, USA

D. Haber · B.W. Hindman

JILA, University of Colorado, Boulder, CO 80309-440, USA

J. Jackiewicz

Astronomy Department, New Mexico State University, P.O. Box 30001, MSC4500, Las Cruces, NM 88003, USA

E. Khomenko

Instituto de Astrofisica de Canarias, 38205, C/Via Láctea, s/n, Tenerife, Spain

R. Komm

National Solar Observatory, Tucson, AZ 85719, USA

P. Rajaguru

Indian Institute of Astrophysics, Bangalore, India

M. Rempel - M.J. Thompson

HAO/NCAR, P.O. Box 3000, Boulder, CO 80307, USA

M. Roth $\cdot$ R. Schlichenmaier

Kiepenheuer-Institut für Sonnenphysik, Schöneckstr. 6, 79104 Freiburg, Germany 


\begin{abstract}
While sunspots are easily observed at the solar surface, determining their subsurface structure is not trivial. There are two main hypotheses for the subsurface structure of sunspots: the monolithic model and the cluster model. Local helioseismology is the only means by which we can investigate subphotospheric structure. However, as current linear inversion techniques do not yet allow helioseismology to probe the internal structure with sufficient confidence to distinguish between the monolith and cluster models, the development of physically realistic sunspot models are a priority for helioseismologists. This is because they are not only important indicators of the variety of physical effects that may influence helioseismic inferences in active regions, but they also enable detailed assessments of the validity of helioseismic interpretations through numerical forward modeling. In this article, we provide a critical review of the existing sunspot models and an overview of numerical methods employed to model wave propagation through model sunspots. We then carry out a helioseismic analysis of the sunspot in Active Region 9787 and address the serious inconsistencies uncovered by Gizon et al. (2009a, 2009b). We find that this sunspot is most probably associated with a shallow, positive wave-speed perturbation (unlike the traditional two-layer model) and that travel-time measurements are consistent with a horizontal outflow in the surrounding moat.
\end{abstract}

\title{
Contents
}

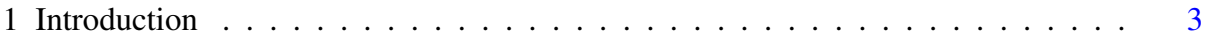

1.1 Why Sunspots Are Interesting . . . . . . . . . . . . . . . . . 3

1.2 The Need for Sunspot Models in Helioseismology . . . . . . . . . . . . . . 4

1.3 What Basic Properties Should be Included in the Models? . . . . . . . . . . . 7

1.4 The Premise of the Article . . . . . . . . . . . . . . . . . . . 7

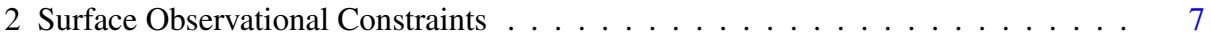

2.1 Sunspot Formation and Evolution . . . . . . . . . . . . . . . . 8

2.2 Sunspot Surface Structure . . . . . . . . . . . . . . . . . . . . . 9

2.3 Sunspot Thermodynamics in the Photosphere . . . . . . . . . . . . . . . . . 12

2.4 The Wilson Depression . . . . . . . . . . . . . . . . . . . . . . . . . . . . . . . . . . .

3 The Deep Structure of Sunspots . . . . . . . . . . . . . . . . . . . . . . . . . . . . . . . . . .

3.1 The Anchoring Problem . . . . . . . . . . . . . . . . . . . . . . . . 14

3.2 The Issue of Flux Emergence . . . . . . . . . . . . . . . . . . . . . . . . . . . . . . . . . . . . . .

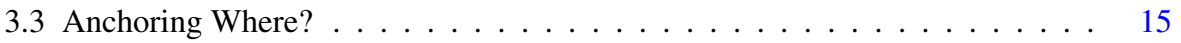

4 Sunspot Models . . . . . . . . . . . . . . . . . . . . . 15

4.1 Semi-Empirical Models of the Sunspot Atmosphere . . . . . . . . . . . . . . . . 15

4.2 Magneto-Hydrostatic (MHS) Models . . . . . . . . . . . . . . . . . 18

H.C. Spruit

Max-Planck-Institut für Astrophysik, Karl-Schwarzschild-Str. 1, 85748 Garching, Germany

K.G. Strassmeier

Astrophysikalisches Institut Potsdam, An der Sternwarte 16, 14482 Potsdam, Germany

M.J. Thompson · S. Zharkov

School of Mathematics and Statistics, University of Sheffield, Houndsfield Road, Sheffield S3 7RH, UK

S. Zharkov

Mullard Space Science Laboratory, University College London, Holmbury St Mary, UK 
4.3 Non-MHS Models . . . . . . . . . . . . . . . . . . . . . 25

4.4 Numerical Simulations of Radiative Magnetoconvection . . . . . . . . . . . 27

5 Diagnostics Potential of Helioseismology . . . . . . . . . . . . . . . . . . . 29

6 Numerical Forward Modeling of Waves Through Model Sunspots . . . . . . . . . 30

6.1 Numerical Methods . . . . . . . . . . . . . . . . . . . . . . 30

6.2 Background Models Stabilized Against Convective Instability . . . . . . . . . 31

6.3 Numerical Codes . . . . . . . . . . . . . . . . . . . . . . . . . . . . . . . . 31

6.4 Eikonal Methods . . . . . . . . . . . . . . . . . . . . . . . . . . . . 34

7 Update on the Analysis of AR $9787 \ldots \ldots \ldots \ldots$. . . . . . . . . . . . . . . . . . . . . . .

7.1 Travel-Times Comparison: Time-Distance and Helioseismic Holography . 35

7.2 Frequency Dependence of One-Way Travel Times . . . . . . . . . . . . . . . . . 35

7.3 Effects of Filtering on Travel Times . . . . . . . . . . . . . . . . . . . . . . 37

7.4 Ring-Diagram Structure Inversions . . . . . . . . . . . . . . . . . . . . . 39

7.5 Moat Flow: Hankel Analysis . . . . . . . . . . . . . . . . . . . . . . . . . . . . . . 42

7.6 Ring-Diagram Analysis of Flows . . . . . . . . . . . . . . . . . . . . . . . . . 42

7.7 Acoustic Halos . . . . . . . . . . . . . . . . . . . . 45

7.8 Comparison of Observed Cross-Covariances with Simulations . . . . . . . . . 47

8 Discussion and Perspective . . . . . . . . . . . . . . . . 48

8.1 Sunspot Structure: A Critical Assessment of Existing Models . . . . . . . . . . . 48

8.2 The Starspot Connection . . . . . . . . . . . . . . . . . . . . . 52

8.3 Conflicting Helioseismic Observations . . . . . . . . . . . . . . . . . . 52

8.4 Emergence of a New Paradigm in Sunspot Seismology . . . . . . . . . . . . . . 54

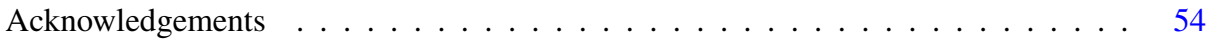

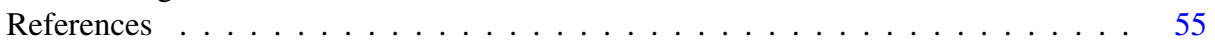

\section{Introduction}

\subsection{Why Sunspots Are Interesting}

Sunspots are the surface manifestations of intense magnetic-flux concentrations that have intersected with the solar surface. As such, they represent one of the major connections of the internal magnetic field of the Sun with its wider environments and are the main sites of solar-activity phenomena. They are at the center of many ongoing challenges in the study of the Sun, as the structure and evolution of sunspots, individually and collectively, are still not fully understood.

Sunspots tend to appear at well-defined latitudes, which vary with the 11-year solar cycle, as summarized in the so-called butterfly diagram. Any theory of the mechanism of the solar global dynamo has to be able to explain this collective behavior. Understanding dynamo processes is of the utmost importance, as they are believed to play crucial roles in many astrophysical phenomena, and sunspots are the best-known candidates to provide us important clues on how they operate.

While the sunspots are easily observed at the surface, determining their subsurface structure is not at all trivial. There are two main hypotheses for the structure of the subsurface magnetic configuration of the spot: the monolithic model (e.g. Cowling, 1946, 1957, 1976) and the jellyfish/cluster/spaghetti model (e.g. Parker, 1975, 1979; Spruit, 1981; Zwaan, 1981). Determining the parameters of these tubes, that is typical size, field strength etc., will help reveal details of the operation of the solar dynamo and how magnetic field is transported up through the convection zone. 
Figure 1 A Hinode G-band image of the well-developed sunspot AR 10953, acquired at 10:10:16 UT, 2 May 2007 (the spatial scale is indicated by the horizontal bar in the upper left). There is a light-bridge close to the northern (upper) tip, and another is beginning to form near the southern edge.

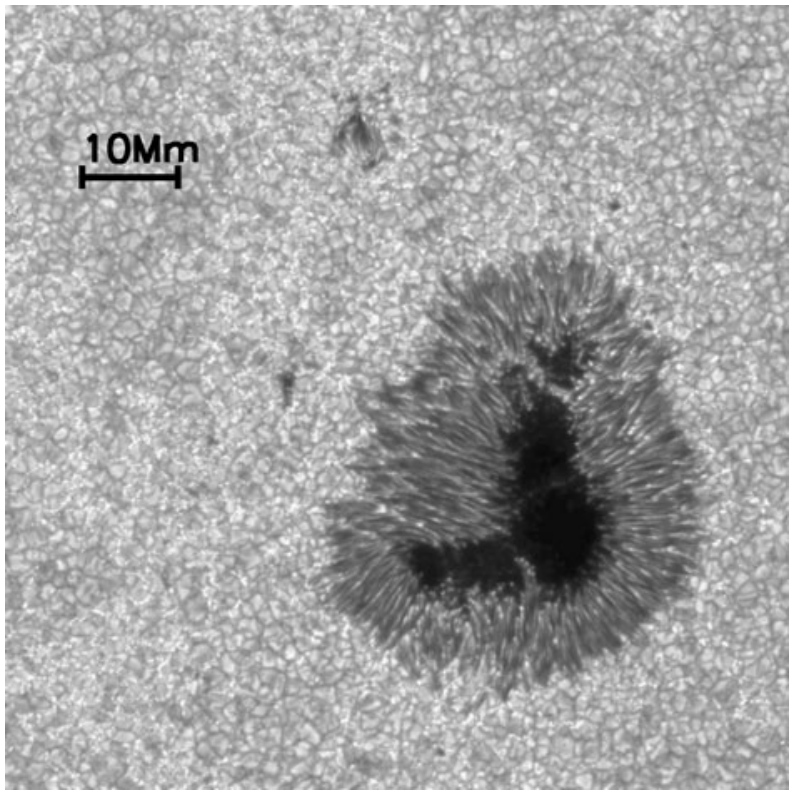

Our understanding of sunspot structure has been somewhat hampered in the past by a lack of high-resolution observations. However, recent advances in adaptive optics, image selection and reconstruction at ground-based telescopes, and the advent of high-resolution space observations with Hinode (see Figure 1), have all led to a wealth of detailed information about the fine structure of sunspot umbrae and penumbrae (see e.g. Scharmer et al., 2002; Thomas and Weiss, 2008). On the other hand, the subsurface structure of sunspots is still poorly understood.

Local helioseismology (e.g. time-distance helioseismology, helioseismic holography, acoustic imaging, ring-diagram analysis; see Gizon and Birch, 2005 and references therein) is the only means by which we can investigate subphotospheric structure. However, interpretations of data have been somewhat ambiguous and inconsistent for applications of local-helioseismic methods in solar active regions. Furthermore, current linear inversion techniques do not yet allow helioseismology to probe the internal structure of sunspots with sufficient accuracy to distinguish between monolith and cluster models. But progress has been made in addressing some of these inconsistencies (e.g. Braun and Birch, 2008; Birch et al., 2009; Gizon et al., 2009a, 2009b) and significant advances have also been made in the simulation of helioseismic wave propagation in magnetized plasmas (see e.g. Cameron, Gizon, and Daiffallah, 2007; Parchevsky and Kosovichev, 2007; Cameron, Gizon, and Duvall, 2008; Hanasoge, 2008; Moradi, Hanasoge, and Cally, 2009; Khomenko et al., 2009; Parchevsky and Kosovichev, 2009; Shelyag et al., 2009; Cameron et al., 2010).

\subsection{The Need for Sunspot Models in Helioseismology}

Sunspot models are important indicators of the variety of physical effects that may influence helioseismic inferences in active regions as well as enabling assessments of the validity of helioseismic inversions. Currently it is also important, in the absence of adequate non-linear inversion techniques, to have models that may be close to the truth as starting points for linear inversions. The associated danger of course is an over-reliance on a small range of 
models that may limit our imagination of what structures may exist and which may bias the helioseismic inversion results.

As illustrated in Figure 2, there are several existing classes of sunspot models - radiative magnetohydrodynamical (MHD) numerical simulations, semi-empirical models, monolithic models, cluster/spaghetti models, self-similar models, potential-field models, current-sheet models, pressure-distributed models, etc. - which are more or less suited to local helioseismology.

The availability of powerful computers has only recently made it possible to produce numerical simulations of sunspots using realistic physics (radiative MHD equations; see Section 4.4). These simulations are more than adequate for studying surface dynamics and fine structure, conducting experiments regarding the subsurface structure, and for providing very useful artificial oscillation data that can be used to test helioseismic inversion methods. Although this is a huge step forward, this approach is unfortunately not suitable for computational helioseismology (where detailed parametric studies need to be conducted using existing numerical wave-propagation codes) for a number of reasons:

i) The subsurface structure of the "realistic" simulations depends on the lower boundary and initial conditions, neither of which is necessarily realistic. The surface properties obtained are more or less correct because the timescales for the evolution at the surface are essentially determined by the radiative-loss term (i.e. convection on the Sun is driven from the surface). The timescale for the subsurface structure of the spot is huge, and is dependent on both the bottom boundary and initial conditions. Nevertheless, work is currently under way to establish whether models with such artificially restrictive lower boundary conditions are able to produce results that are compatible with helioseismic measurements.

ii) The cumbersome computational requirements of such ab-initio simulations. As an indication, simulating the two-hour evolution of a pair of sunspots in Rempel et al. (2009b) takes a number of weeks on extremely powerful supercomputers.

iii) Such simulations still do not address the question of the nature of the deep structure of sunspots. While one may ask whether a sunspot model with the magnetic field clamped $10 \mathrm{Mm}$ below the photosphere is, or is not, compatible with helioseismic measurements, probing down to these depths, however, will first require that we adequately model (and remove) the effects of the surface layers.

Examining a number of different sunspot models is not only essential to test the robustness of helioseismic inferences, but it is also an indispensable need in computational helioseismology where effective inference by matching wave-field simulations to observations relies on the availability of sunspot models. For example, sunspot models with very different subsurface configurations (e.g. cluster or monolith, connected or disconnected, etc.) can be used to verify what helioseismic signatures would be observed for a sunspot with that particular subsurface structure. It is also essential to be able to continually tune the surface parameters of the model to actual observations in order to match the wave-field signatures observed. So the aim here is not to have the best sunspot model, but to have a group of models that can be useful for computational helioseismology - models that can be tuned to match the non-helioseismic observations, and then in turn be used in inversions to determine the subsurface structure. Of course, with multiple models the inversions are unlikely to be unique. Therefore, such parametric studies will provide us with an idea of what is, and what is not, reliable in the inversions. As well as more or less realistic models, highly simplified models which isolate just a few physical effects can also be useful for elucidating the sensitivity of inference methods in helioseismology to particular effects. Nonetheless, it must be 


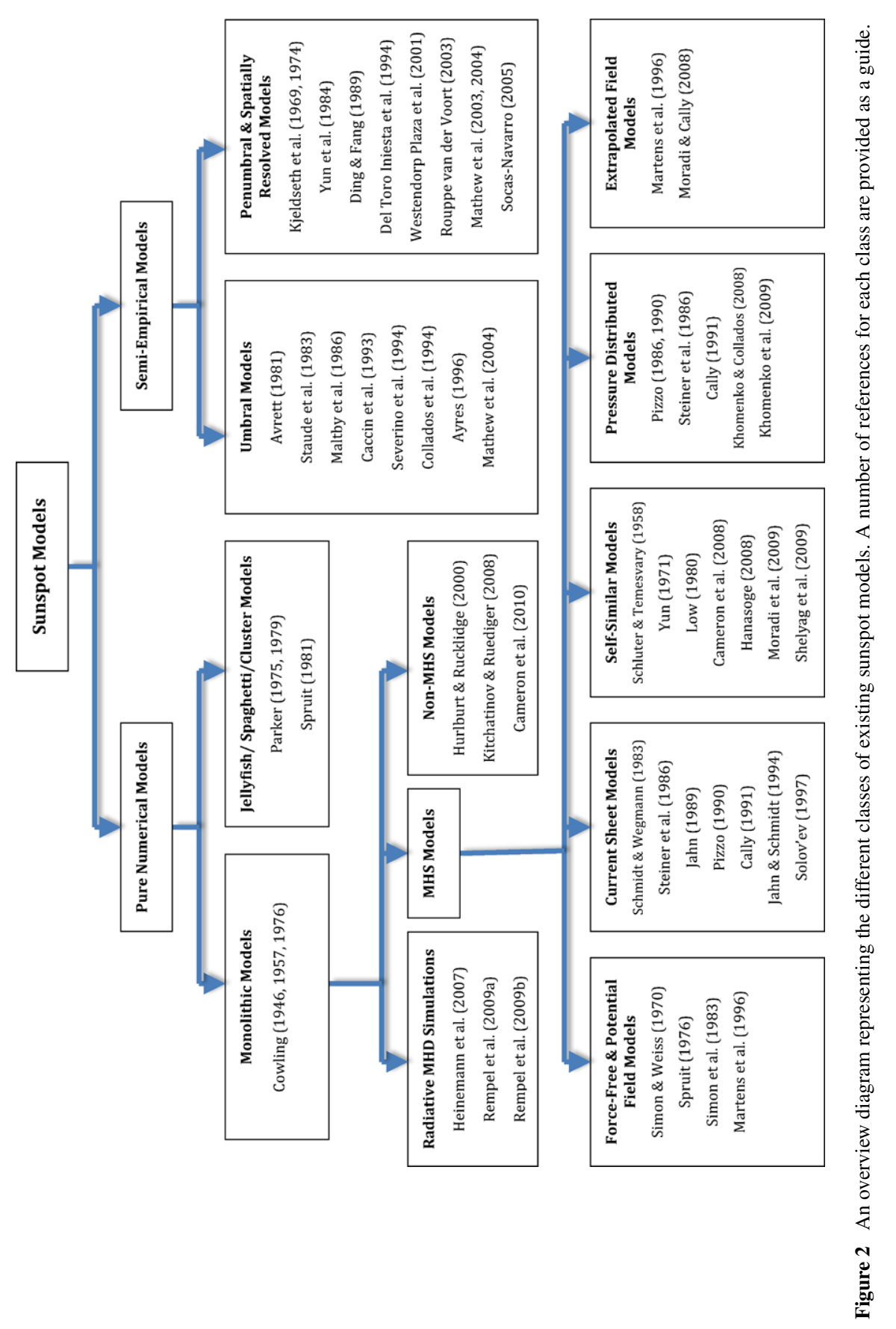


borne in mind if using such models that other physical effects may have seismic signatures that are qualitatively or quantitatively similar.

\subsection{What Basic Properties Should be Included in the Models?}

Apart from the desired characteristics mentioned above, models of the gross magnetic structure of sunspots for use in computational helioseismology should ideally posses a high degree of flexibility and computational efficiency to allow for extensive parameter studies using existing numerical simulation codes. A number of other essential, observationally derived, characteristics should also be embodied by the models. For example, an accurate prescription for the surface (photospheric) magnetic-field characteristics (of both the umbra and penumbra, e.g. field strength, orientation, twist, return flux, etc., see Section 2.2.1 and Section 2.2.2). These should comply with observations and, ideally, one should be able to model the sunspot field on extrapolations from observed surface magnetic profiles (e.g. vector magnetograms). One should also be able to choose the profile of thermodynamic parameters (pressure, density, temperature, etc., see Section 2.3 and Section 4.1) in the umbra and penumbra from either spectro-polarimetric inversions or semi-empirical models of the solar atmosphere. Some important dynamical phenomena (e.g. the Evershed flow, moat flow, etc.; see Section 2.2.4 and Section 2.2.5) should also be taken into account, while a realistic and consistent (e.g. Section 2.4) description of the Wilson depression is also essential.

\subsection{The Premise of the Article}

The basic premise of this article is to satisfy two complementary goals: The first goal is to present a critical review of the existing physical models for the subsurface structure of sunspots, in the context of local helioseismology and numerical simulations of wave fields, and magnetic field - wave interaction. As discussed above, physical sunspot models are critically important to assess the validity of the helioseismic inversions. In addition, numerical simulations of the propagation of solar waves through model sunspots are emerging as a valid and realistic technique to interpret helioseismic data. The success of this approach relies on a very close interaction between sunspot modelers and helioseismologists.

The second goal is to extend the helioseismic analysis undertaken by Gizon et al. (2009a, 2009b) of the sunspot in Active Region (AR) 9787, which was the topic of the Third HELAS (European Helio- and Asteroseismology Network) Local Helioseismology Workshop, held in Berlin on 12 - 15 May 2009. This sunspot was observed during the period 20 - 28 January 2002 by the SOHO/MDI instrument. Serious inconsistencies between the different helioseismic methods were uncovered, which cannot be left unanswered.

\section{Surface Observational Constraints}

In this section we briefly review some of the main observational characteristics of sunspot formation and evolution. The aim here is to present a very general overview of some of the pertinent issues related to sunspot observations that need to be considered when developing a realistic sunspot model. Comprehensive reviews by Solanki (2003), Thomas and Weiss (2004), Tobias and Weiss (2004), Schlichenmaier (2009), and the books by Thomas and Weiss (1992, 2008) (and references therein), have excellent extended discussions on both the observational and theoretical aspects of sunspots and active regions. 


\subsection{Sunspot Formation and Evolution}

\subsubsection{Flux Emergence}

Individual bundles of magnetic flux are believed to rise from deep in the convection zone and break through the surface of the Sun. As they approach the surface, each flux bundle is shredded into many separate strands which, upon emergence, are quickly concentrated into small, intense ( $\mathrm{kG}$ strength) magnetic flux bundles (or elements) by the vigorous convection occurring in the thin superadiabatic layer at the top of the convection zone. These small flux elements then accumulate at the boundaries between granules or supergranules, and some of them coalesce to form small pores (Keppens and Martínez Pillet, 1996; Leka and Skumanich, 1998). Some pores and flux elements in turn coalesce to form sunspots.

A number of studies have examined the buoyant rise of magnetic flux from just below the surface and rising into a non-magnetized atmosphere (e.g. Magara and Longcope, 2001, 2003; Manchester et al., 2004; Cheung, Schüssler, and Moreno-Insertis, 2007). A number of studies modeling the rise of thin flux tubes through the convection zone have also shown that the tubes must have a significant amount of twist in order to maintain their integrity and not fragment in the face of hydrodynamic forces, and indeed observations show that magnetic flux usually emerges at the surface already in a significantly twisted state (e.g. Riethmüller et al., 2008).

\subsubsection{Sunspot Formation and Decay}

When the flux does emerge, it is often in the form of pore structures of the order of a Mm or so in size (Vrabec, 1974; Zwaan, 1978, 1992; McIntosh, 1981). Pores have continuum intensities ranging from $80 \%$ down to $20 \%$ of the normal photospheric intensity, with maximum magnetic-field strengths of 1500-2000 G. If a growing pore reaches a sufficient size (a diameter of about $3500 \mathrm{~km}$, but sometimes as much as $7000 \mathrm{~km}$ ) or a sufficient total magnetic flux of order $10^{20} \mathrm{Mx}$ (Leka and Skumanich, 1998), and if the magnetic field reaches an inclination from the vertical that is greater than about $\gamma=35^{\circ}$ (Martínez Pillet, 1997), then it forms a penumbra at its periphery and becomes a fullfledged sunspot. The formation of a penumbra is a rapid event, occurring in less than 20-30 minutes, and the characteristic sunspot magnetic-field configuration and Evershed flow are both established within this same, short time period (Leka and Skumanich, 1998; Yang et al., 2003). Furthermore, the fact that the largest pores are observed to be bigger than the smallest sunspots also provides evidence that the pore-sunspot transition is associated with hysteresis (Bray and Loughhead, 1964; Rucklidge, Schmidt, and Weiss, 1995).

The time scale for the formation of a large sunspot is between a few hours and several days. A sunspot can span a lifetime of months, but more typically of weeks (Solanki, 2003). However, this life expectancy is considerably shorter than the magnetic diffusion time $\left[t_{\mathrm{D}}=\right.$ $l^{2} / \eta$ where $l$ is the width of the current sheet and $\eta=1 / \sigma$ is the magnetic diffusivity] across a solar active region where estimates for $t_{\mathrm{D}}$ range from hundreds to thousands of years $(e . g$. Priest and Forbes, 2000). This reduced lifetime suggests that a convective instability sets in that enhances the decay process, via fragmentation. Another possible process is the action of turbulent diffusion, owing to the non-linear dependence of the diffusivity on the strength of the magnetic field (Petrovay and Moreno-Insertis, 1997). An overview of sunspot decay was presented by Martínez Pillet (2002). 


\subsubsection{Surface Evolution}

Observational evidence indicates a significant change in the dynamical properties of sunspots and active regions from "active" to "passive" evolution shortly after their emergence (Schüssler, 1987; Schrijver and Title, 1999; Schüssler and Rempel, 2005). Initially, the emerging magnetic flux displays the characteristics of a rising, fragmented flux tube and evolves according to its internal large-scale dynamics (e.g. McIntosh, 1981; Strous et al., 1996). Within a few days after emergence, the proper motion of the sunspots with respect to the surrounding plasma begins to decay. Larger magnetic structures also start to fragment into small-scale flux concentrations, which are largely dominated by the local near-surface flows (granulation, supergranulation, differential rotation, meridional circulation). The magnetic flux is then passively advected by these velocity fields, gradually dispersing over a large area. This process is efficiently described by well-established surface flux-transport mechanisms which model the evolution of the magnetic field at the solar surface (e.g. Wang, Nash, and Sheeley, 1989; van Ballegooijen, Cartledge, and Priest, 1998; Schrijver, 2001; Baumann et al., 2004).

\subsection{Sunspot Surface Structure}

\subsubsection{Field Strength and Orientation}

A sunspot's maximum magnetic-field strength tends to increase approximately linearly with sunspot diameter, from around $2000 \mathrm{G}$ for the smallest to $4000 \mathrm{G}$ or more for the largest sunspot (e.g. Ringnes and Jensen, 1960; Brants and Zwaan, 1982; Kopp and Rabin, 1992; Collados et al., 1994; Livingston, 2002; Livingston et al., 2006). The field strength drops steadily toward the sunspot's periphery, becoming $700-1000 \mathrm{G}$ at the edge of the visible sunspot (e.g. Mathew et al., 2004).

The field strength decreases with height within the visible outline of the spot. At photospheric levels in the umbra, line-of-sight field strength decreases of $\approx 1-3 \mathrm{G} \mathrm{km}^{-1}$ are observed (Balthasar and Schmidt, 1993; Schmidt and Balthasar, 1994), but when averaged over a height range of $2000 \mathrm{~km}$ or more, the field strength gradient reduces to 0.3 $0.6 \mathrm{G} \mathrm{km}^{-1}$ (Solanki, 2003). The strongest field within a sunspot is usually associated with the darkest part of the umbra and is generally close to vertical. At the visible outer sunspot boundary it is inclined by $70-80^{\circ}$ to the vertical (Bellot Rubio et al., 2003a; Mathew et al., 2004) if one applies inversions that assume a constant field inclination in the atmosphere. Assuming more than one magnetic component or gradients along the line of sight, high-resolution polarimetric studies present evidence for magnetic flux that returns to the surface in the outer penumbra (e.g. Westendorp Plaza et al., 2001; Bellot Rubio, Balthasar, and Collados, 2004; Borrero et al., 2004, 2006; Langhans et al., 2005; Ichimoto et al., 2007, 2009; Beck, 2008; Jurčák and Bellot Rubio, 2008), i.e. there is evidence that a fraction of the sunspot magnetic field does not extend into the chromosphere, but submerges beneath the photosphere in the outer penumbra.

\subsubsection{Field Twist}

As we have already mentioned, it appears that at least a small amount of twist is needed in a rising flux tube in order for it to resist fragmentation and preserve its identity as it rises through the convection zone; hence systematic twists of sunspot fields are relevant to the emergence and stability of sunspot magnetic fields. Observations also indicate that 
the magnetic field of regular sunspots can be twisted, with an azimuthal twist $\phi \approx 10^{\circ}-35^{\circ}$ (Hagyard, West, and Cumings, 1977; Gurman and House, 1981; Lites and Skumanich, 1990; Skumanich, Lites, and Martínez Pillet, 1994; Westendorp Plaza et al., 2001).

However, the more recent observations of Mathew et al. (2003) indicate that for regular isolated sunspots, the global azimuthal twist of the field does not significantly exceed $20^{\circ}$. Moreover, Yun (1971) and Osherovich and Flaa (1983) have included the effects of an azimuthal field twist in their (self-similar) sunspot models and find that the introduction of a moderately twisted field, compatible with observations, contributes little to the force balance in spots and only slightly changes the main characteristics of their sunspot models (e.g. the mixing-length parameter, effective temperature, Wilson depression, and the central field strength remain practically the same). This is not surprising, in view of the fact that the measured $B_{\psi}$ from observations is small compared to $B_{z}$ and $B_{r}$ over most of the sunspot region.

\subsubsection{Umbral Dots}

Umbral dots, bright dot-like feature observed inside an umbra, are found in almost all sunspots and also in pores (Sobotka, 1997, 2002). They are observed to cover only 3 $10 \%$ of the umbral area but contribute $10-20 \%$ of the total umbral brightness (Sobotka, Bonet, and Vazquez, 1993). Their distribution is not uniform: they can occur in clusters and alignments, and no large dots are found in dark nuclei (Rimmele, 1997).

On average, umbral dots are $500-1000 \mathrm{~K}$ cooler than the photosphere outside a spot, but about $1000 \mathrm{~K}$ hotter than the coolest parts of the umbra itself (Kitai et al., 2007). The magnetic field in umbral dots appears to be weaker than that in the umbral background (Sobotka, 1997). Socas-Navarro et al. (2004) found differences of several hundred gauss and deduced that the fields were more inclined to the vertical, by about $10^{\circ}$. Furthermore, a small upward velocity of $30-50 \mathrm{~m} \mathrm{~s}^{-1}$ and $200 \mathrm{~m} \mathrm{~s}^{-1}$ has been reported in umbral dots relative to the umbral background by Rimmele (1997) and Socas-Navarro (2002).

Schüssler and Vögler (2006) have presented realistic numerical simulations of umbral magnetoconvection in the context of the monolithic model, by assuming an initially uniform vertical magnetic field. Their model reproduces all of the principal observed features of umbral dots, including their dark lanes (Rimmele, 2008). Their results provide support for the monolithic model, demonstrating that umbral dots can arise naturally as a consequence of magnetoconvection in a space-filling vertical magnetic field. The more recent numerical simulations of Heinemann et al. (2007) and Rempel, Schüssler, and Knölker (2009a) also confirm this.

\subsubsection{Evershed Flow}

The Evershed flow is observed as an outward-directed (horizontal) flow observed in the photospheric layers of penumbrae. The inverse Evershed flow is an inward-directed flow in chromospheric layers. A number of well-established observational properties of the Evershed flow are:

- The averaged flow velocity increases from the inner to the outer penumbra. From observed line asymmetries it is concluded that the flow is located in the deep photosphere (Maltby, 1964; Schlichenmaier, Bellot Rubio, and Tritschler, 2004).

- Observed velocities of the flow typically exceed $6 \mathrm{~km} \mathrm{~s}^{-1}$ (Rouppe van der Voort, 2002; Bellot Rubio et al., 2003a) in the outer penumbra, but can also exceed $10 \mathrm{~km} \mathrm{~s}^{-1}$ in localized patches (e.g. Bellot Rubio, Balthasar, and Collados, 2004). 
- The direction of the Evershed flow is close to horizontal. Azimuthal averages reveal that the flow angle varies from $70^{\circ}$ (average flow points upward) in the inner penumbra to some $100^{\circ}$ (flow points downward) in the outer penumbra (Schlichenmaier and Schmidt, 2000).

- The Evershed flow is magnetized. This is obvious from Stokes $V$ profiles with more than two lobes. These additional lobes are Doppler shifted (e.g. Schlichenmaier and Collados, 2002).

Two models have been proposed to explain a number of observational properties: the "siphon-flow" model as proposed by Montesinos and Thomas (1997), and the "movingtube" model by Schlichenmaier, Jahn, and Schmidt (1998). Montesinos and Thomas (1997) elaborated on the idea of Meyer and Schmidt (1968) that the flow is driven by a gas-pressure difference between the footpoints of a thin magnetic-flux tube in magnetohydrostatic (MHS) equilibrium. On the other hand, Schlichenmaier, Jahn, and Schmidt (1998) developed a dynamical 2D model of a thin magnetic-flux tube that acts as a convective element in a superadiabatic and magnetized penumbral atmosphere. In their model, the convective rise of the thin flux tube to the surface initiates a local pressure-gradient build up, leading to a gas flow along the tube. Penumbral grains are then identified as the hot upflow locations where the gas reaches the (optical) surface.

Numerical simulations of radiative magnetoconvection in inclined magnetic fields (e.g. Heinemann et al., 2007; Scharmer, Nordlund, and Heinemann, 2008; Rempel, Schüssler, and Knölker, 2009a; Rempel et al., 2009b) are only beginning to reproduce the structure of the outer penumbra, with its horizontal and returning magnetic fields and fast Evershed flows along arched channels. They have already succeeded in reproducing single elongated filaments with lengths of up to a few Mm, which resemble in many ways what is observed as thin light bridges and penumbral filaments of the inner penumbra. In their simulations, they find that the progression of the filament heads toward the umbra during their formation phase is not caused by the inward motion of a narrow flux tube, but rather due to the expansion of the sheet-like upflow plumes along the filament.

\subsubsection{Moat Flow and Moving Magnetic Features}

The moat flow is an outflow that initiates immediately after the formation of a penumbra. Moats are typically 10 to $20 \mathrm{Mm}$ wide, with the outer radius of the moat appearing to scale with the size of the enclosed sunspot, being about twice the radius of the spot itself (Brickhouse and Labonte, 1988). The moat-flow velocity is about 0.5 to $1 \mathrm{~km} \mathrm{~s}^{-1}$, and can be seen by proper motions of granules as well as by Doppler-shift measurements (Balthasar et al., 1996). The flow usually persists over the duration of the spot's life, while the area and magnetic flux of the sunspot decrease at a roughly constant rate. As the moat flow evolves, it pushes the magnetic flux to its periphery, leaving the moat largely free of magnetic field except for small magnetic features (known as moving magnetic features) that move outward across the moat at speeds of about $1 \mathrm{~km} \mathrm{~s}^{-1}$ (Sheeley, 1969, 1972; Vrabec, 1971, 1974; Harvey and Harvey, 1973; Brickhouse and Labonte, 1988; Wang and Zirin, 1992; Yurchyshyn, Wang, and Goode, 2001; Sainz Dalda and Bellot Rubio, 2008a).

The moat flow is only present in the presence of a penumbra. Pores that have no penumbra also lack the moat flow. Observations of irregular sunspots by Vargas Domínguez et al. (2007) indicate that the moat flow exists only on sunspots sides where a penumbra has formed. On sunspot sides where the umbra and the granulation are adjacent, no moat flow is detected. Moreover, in such irregular configurations moat flows are only observed as radial extensions of penumbral filaments, but not perpendicular to the filament. 
The moat flow has also been detected through local helioseismology, using $f$-mode time - distance helioseismology. Using azimuthally averaged MDI data, Gizon, Duvall, and Larsen (2000) find an outflow extending well beyond the sunspot boundary (up to $30 \mathrm{Mm}$ ) which reaches a peak of $1 \mathrm{~km} \mathrm{~s}^{-1}$ just outside the penumbra. This flow is consistent with the moat flow. In another recent study, Gizon et al. (2009a, 2009b) used $f$ to $p_{4}$ ridge-filtered time-distance travel times to produce linear inversion for flows around AR 9787. These inversions showed an azimuthally averaged horizontal outflow in the first $4 \mathrm{Mm}$ beneath the surface, reaching an amplitude of $230 \mathrm{~m} \mathrm{~s}^{-1}$ at a depth of $2.6 \mathrm{Mm}$ and radial distance of some $5 \mathrm{Mm}$ outside the outer spot boundary. The inversion results were in line with observations of the moat flow in AR 9787 presented by Gizon et al. (2009a, 2009b), the strength and extent of which was characterized by measuring the observed motion of the moving magnetic features (MMFs) using a local correlation-tracking method. Their measurements indicated a peak amplitude of $230 \mathrm{~m} \mathrm{~s}^{-1}$ for the moat flow, extending out to about $45 \mathrm{Mm}$ from the spot center.

As the moat flow is unmagnetized and has velocities that are much smaller than the Evershed flow, the link between the two is not obvious. One possibility is that the gas pressure that builds up beneath the penumbra could drive the moat flow (beneath the penumbra the magnetopause is largely inclined). Sainz Dalda and Bellot Rubio (2008b) detect bipolar MMFs within the penumbra, which migrate outward into and throughout the moat. This is consistent with a scenario proposed by Schlichenmaier (2002): a magneto-convective overshoot instability in an Evershed flow channel leads to a bipolar MMF that travels outward along the magnetic canopy.

\subsection{Sunspot Thermodynamics in the Photosphere}

There are a number of semi-empirical and observational models as reference, consisting of both one- and two-component models, for the umbra and for the penumbra (see Section 4.1 for a more detailed discussion). The basic assumption underlying almost all singlecomponent models is that it is possible to describe all umbrae (or at least those above a certain size) by a single thermal model.

Thus, a very important question in this context is whether sunspot brightness (temperature) or magnetic-field strength actually varies with the size of the sunspot. Theoretical models of sunspots based on MHS equilibrium and inhibition of convective heat transport (e.g. Deinzer, 1965; Yun, 1970) typically obtain lower temperatures for stronger magnetic fields. However, observations by Rossbach and Schröter (1970) and Albregtsen and Maltby (1981) indicated no dependence of umbral intensity on umbral size for umbral diameters greater than about $8^{\prime \prime}$. On the other hand, the observations of Kopp and Rabin (1992) showed a nearly linear decrease in umbral brightness with umbral radius for six sunspots. A similar result was obtained for seven spots at visible wavelengths by Martínez Pillet and Vazquez (1993) and Collados et al. (1994). This was also confirmed in a more recent study of continuum images of more than 160 sunspots taken during Solar Cycle 23 by Mathew et al. (2007). Indeed, Norton and Gilman (2004) use this dependence to predict the peak field strength of a sunspot from its brightness to an accuracy of about $100 \mathrm{G}$. Hence, even though the relatively homogeneous umbral nuclei cannot be described by a single universal atmosphere, these models may nonetheless be used as boundary conditions in theoretical global sunspot models.

Another related question is whether the umbra-photosphere brightness ratio of large sunspots varies over the solar cycle. Albregtsen and Maltby $(1978,1981)$ find that sunspots were darkest at the beginning of Sunspot Cycle 20 and that spots appearing later in the cycle were progressively brighter (with a nearly linear dependence on the phase of the cycle), 
up until the new Cycle 21 spots appeared, which were again darkest. Subsequent observations showed that the same behavior occurred in Cycle 21 (Maltby et al., 1986). Penn and Livingston (2006) found similar behavior during Cycle 23. However, the results of Mathew et al. (2007) find no significant change in umbral brightness over Cycle 23.

\subsection{The Wilson Depression}

The reduced opacity in a sunspot, and the consequent depression of the $\tau=1$ level, arises mainly from two effects: $i$ ) the reduced temperature in the spot atmosphere leads to a decrease in the $\mathrm{H}^{-}$bound-free opacity, and ii) the radial force balance (including magnetic pressure and curvature forces) demands a lower gas pressure within the spot, further reducing the net opacity.

A purely observational determination of the Wilson depression is complicated by evolutionary changes in the shape of the spot. Estimates from observations range from $400-1500 \mathrm{~km}$ for mature spots (Bray and Loughhead, 1964; Gokhale and Zwaan, 1972; Balthasar and Wöhl, 1983). Martínez Pillet and Vazquez (1993) assume a linear relation between magnetic pressure and temperature in a spot (as indicated by observations), in which case the radial force balance yields a simple relationship between the net magnetic curvature force and the Wilson depression. They computed a Wilson depression of 400-800 km in the umbra of their observed sunspot (the range being due to different assumed values of the curvature forces). Solanki, Walther, and Livingston (1993) and Mathew et al. (2004) used this approach to determine the variation of the Wilson depression with radius across a sunspot. They found values of some $100 \mathrm{~km}$ in the penumbra and some $400 \mathrm{~km}$ in the umbra, with a fairly sharp transition at the umbra-penumbra boundary. In a more recent study, Watson et al. (2009) compared observations of sunspot longitude distribution and Monte Carlo simulations of sunspot appearance using different models for spot growth rate, growth time and depth of Wilson depression, deducing a mean depth for the umbral $\tau=1$ layer of $500-1500 \mathrm{~km}$.

\section{The Deep Structure of Sunspots}

Though the small-scale structure seen at the surface of a sunspot is not exactly in equilibrium, the spot itself survives on much longer time scales than would be expected if it were a superficial structure. It lives much longer than the time scale on which it would evolve if it were not in a stable equilibrium, which is the time for the Alfvén speed to cross the spot (on the order of an hour).

The magnetic field of a spot cannot be just a surface phenomenon, however, since magnetic-field lines have no ends. The extension of the spot's field lines above the solar surface can be observed in the chromosphere and corona, but they must continue below the surface as well. In contrast with a scalar field such as pressure, the magnetic field of a sunspot cannot be kept in equilibrium simply by pressure balance at the surface: the tension in the magnetic-field lines continuing below the surface exerts forces as well. The question of spot equilibrium thus involves deeper layers, down to wherever the field lines continue. This is the well-known "anchoring" problem of sunspots (Parker, 1979): at which depth, and by which agent is the sunspot flux bundle kept together? 


\subsection{The Anchoring Problem}

As an answer to this problem, Parker (1979) postulates the existence of a horizontal flow at some depth below the surface, converging on and flowing through the magnetic-flux bundle of the spot. The drag force of this flow on the field would prevent the bundle from fragmenting. There are some obstacles to this idea. A horizontal flow is observed around spots (the moat flow), but it is of the opposite sign to the proposed inflow. There is no theory for the cause of the proposed flow of Parker (1979). In fact, the "heat-flux blocking" by the sunspot would cause a flow of opposite sign, and this is actually observed in the form of the moat flow. It is also questionable if the proposed flow would actually be sufficient to keep the flux bundle together, in view of the (interchange) instabilities to be expected in this picture (Schüssler, 1984). What has kept Parker's proposal alive, however, is the helioseismic inference (Duvall et al., 1996) of a huge downflow of $2 \mathrm{~km} \mathrm{~s}^{-1}$ of the sign proposed by Parker. It is a puzzling observation, which, if confirmed, would require new theoretical ideas. However, more recent helioseismic inferences derived from inversions of subsurface flows around the sunspot in AR 9787 (see Section 7.6.2) do not confirm the existence of this downflow.

A second proposal (already implicit in Cowling, 1953, and developed by Babcock, 1963; Leighton, 1969; Spruit and Roberts, 1983) is that the flux bundle of a spot actually continues all of the way to the base of the convection zone, to the layer of toroidal field from which the active region erupted. At this depth, a field strength of $\approx 80 \mathrm{kG}$ is inferred from flux-tube emerging calculations (see e.g. D'Silva and Choudhuri, 1993; Caligari, Moreno-Insertis, and Schüssler, 1995). The Alfvén travel time for a change at the base to propagate to the surface (more accurately: the propagation time of a transverse tube wave) is then about five days. This is the time scale on which the spot would change if there were nothing to maintain conditions at the base. The idea is thus that a spot, while in equilibrium at the surface, would actually be a transient structure in the layers from which its magnetic field originates. This proposal agrees roughly with the lifetime of most small spots. It is too short, however, to explain the anchoring of stable, long-lived spots.

\subsection{The Issue of Flux Emergence}

The anchoring problem of long-lived spots is thus still somewhat open. Quite clear, however, is the picture of flux emergence: the process by which a loop of flux rises from a horizontal layer of magnetic flux at the base of the convection zone to form an active region, as discussed briefly in Section 2.1.1. Simplified 1D calculations in the "thin tube" approximation (D'Silva and Choudhuri, 1993; Fan, Fisher, and McClymont, 1994; Caligari, Moreno-Insertis, and Schüssler, 1995) point to a field strength of about $10^{5} \mathrm{G}$ at the base of the convection zone. At this strength, agreement is reached with three independent key properties of active regions: $i$ ) the time scale of emergence (a few days), ii) the heliographic-latitude range of emergence, and iii) the tilt of active-region axes (e.g. Caligari, Schüssler, and Moreno-Insertis, 1998, and references therein). This is also the field strength at which the horizontal field is first expected to become unstable to the development of bends in the field lines, creating loops rising to the surface (Schüssler et al., 1994).

However, the picture presented by rising flux-tube simulations is somewhat incomplete, since the last stages of the flux-emergence process in the near-photospheric layers are typically excluded. It has been pointed out by Schüssler and Rempel (2005) that the typical size of active regions corresponds to a wavenumber of $m=10$ to 60 , while buoyant flux tubes typically prefer $m=1$ or 2 instabilities. From this mismatch in scales one would expect an 
unrealistic drift of sunspots apart from each other much further than actually observed due to magnetic tension forces. Possible solutions could include a "dynamical disconnection" as suggested by Schüssler and Rempel (2005) or a flux-emergence process that prefers larger wave numbers from the beginning. Observational evidence for this process has recently been provided by Svanda, Klvaňa, and Sobotka (2009).

Overall, the convergence of these lines of evidence supports the basic correctness of the flux-emergence picture of active-region formation that has long been intuitively evident from observations (Cowling, 1953). It has the important implication that the energy density of the magnetic field at its source is more than two orders of magnitude larger than energy density in convective flows; at such a strength the field is nearly unaffected by convective flows. The same is the case at the surface of a sunspot. This is incompatible with the cornerstone of traditional mean-field dynamo models based on the effect of convective turbulence acting on magnetic fields. The picture sketched above and the mean-field convective dynamo model thus cannot both be correct. This fact is remarkably politely ignored in discussions on theories of the solar cycle.

\subsection{Anchoring Where?}

Some details of the evolution of an active region give additional clues about the anchoring of sunspots. After their formation, long-lived spots wander about a bit in longitude and latitude (e.g. like AR 9787, Gizon et al., 2009a, 2009b), settling to their final position over the course of a few days (e.g. Mazzucconi, Coveri, and Godoli, 1990). This is similar to the inferred Alfvén travel time from the base of the convection zone and the surface. The observed settling process thus agrees with the notion of anchoring in deep layers. The "settling time scale", the Alfvén travel time from the anchor to the surface, agrees with the field strength at the base of the convection zone inferred from the other properties of the emergence process mentioned above.

While anchoring at the base of the convection zone thus agrees with a range of observational indications, this does not in itself prove that other locations within the convection zone are excluded. Such locations are, however, somewhat unlikely. Apart from the boundary with the stably stratified interior, it is hard to find a plausible location for anchoring a magnetic field in a convecting stellar envelope, which is itself unstable to fluid displacements. The anchor of a long-lived spot needs to act over a time longer than the convective turnover time of the envelope as a whole (which would be on the order of a month, in the classical mixing length view).

\section{Sunspot Models}

\subsection{Semi-Empirical Models of the Sunspot Atmosphere}

Semi-empirical models of umbral or penumbral atmospheres give the variation of thermodynamic variables and magnetic-field vector with optical depth based on empirical data and theoretical considerations of mechanical equilibrium and radiative transfer. Semi-empirical models can be divided into two groups, based on the observational material and methods used for their derivation.

One group consists of spatially unresolved, one-dimensional models based on multiwavelength observations of the continuum, weak and strong spectral lines. The general procedure in constructing a model atmosphere usually involves first determining a temperatureoptical depth relation, as a best fit to the empirical data, and then determining the gas and 
electron pressures by integrating the equation of hydrostatic equilibrium. In general, a number of assumptions/generalizations are made when calculating this type of semi-empirical models:

i) For the lower photospheric layers, measurements of the center-to-limb variation of continuum intensity at several spectral intervals and the profiles of the weak spectral lines (or the wings of strong lines) are used together with the LTE assumption. For the high chromospheric layers, strong spectral lines are used such as $\mathrm{Ca}$ II $\mathrm{H}$ and $\mathrm{K}$ and IR lines, $\mathrm{H} \alpha, \mathrm{Na}$ I D, etc. and the analysis is carried out in NLTE.

ii) Hydrostatic equilibrium is assumed. The question of the magnetic-field distribution is not addressed.

Most models consist of a single component, meant to represent a horizontal average over the umbra or penumbra, while a number of models consist of two components, designed to treat bright and dark regions separately in observations that do not fully resolve them spatially.

Another group of models uses a relatively new strategy based on the inversion of the polarized light radiative-transfer equation (e.g. Ruiz Cobo and del Toro Iniesta, 1992). The input data for these models are spectro-polarimetric or spectral observations (typically of high resolution). Formal mathematical methods are used to find the best fit to the spectralline profiles and only a few spectral lines are required to produce a model atmosphere. This method provides sunspots models (umbra, penumbra, and the surrounding plage) spatially resolved to the extent allowed by the observations. The following assumptions are typical:

i) The inversion of the radiative transfer equation is done under LTE conditions for photospheric lines. NLTE inversion results have been reported for the chromospheric Ca II infrared triplet (Socas-Navarro, 2005). All variables are derived as a function of optical depth.

ii) The gas stratification is obtained at heights of formation of spectral lines, at most $\approx 120 \mathrm{~km}$ below, and up to $\approx 300-400 \mathrm{~km}$ above the continuum level. The data about the chromospheric layers are less frequent (e.g. Socas-Navarro, 2007).

iii) The models include magnetic-field vector and line-of-sight velocity component with or without gradients.

iv) For practical reasons, the atmosphere is assumed to be in MHS equilibrium at each spatial location.

v) The Wilson depression is not an inherent part of the models, but instead must be determined from additional considerations (e.g. lateral pressure balance).

Such semi-empirical models can include one, two, or more magnetic components describing the variations of thermodynamic parameters, velocities, and magnetic-field vector at each individual spatial location, supposing these variations are not completely resolved by observations. The shapes of the polarization profiles in the penumbra suggest that they result from at least two different magnetic components, one of them carrying the Evershed flow (Bellot Rubio, 2003). Note that the magnetic-field strength and its inclination in the penumbra are correlated with the brightness of the penumbral filaments (Beckers and Schröter, 1969; Schmidt et al., 1992; Title et al., 1992; Bellot Rubio, 2003; Solanki, 2003), thus the components with different field strengths also typically have different temperatures.

All of these models are useful in constraining certain physical processes that determine the structure of a real sunspot atmosphere, and also in providing a background model for studies of element abundances, wave propagation, and other behavior in a sunspot. 


\subsubsection{Umbral Models}

Below is a summary of some of the more often used umbral models. The first three belong to the first group of models described above and only provide variations of thermodynamical quantities.

- Avrett (1981): Provided a combined model of the umbral photosphere, chromosphere, and transition zone, by combining the low photospheric model of Albregtsen and Maltby (1981) with the upper photospheric and chromospheric parts of the Lites and Skumanich (1982) model and the transition-region model of Nicolas et al. (1981).

- Staude et al. (1983): Derived a comprehensive umbral model covering the full height range from the photosphere to the corona. Their model was based on a large set of observations (radio, optical, EUV, X-ray). The photospheric structure was taken largely from Stellmacher and Wiehr (1975), while its lower chromospheric stratification is similar to that of Teplitskaya, Grigor'eva, and Skochilov (1977). They also introduced two components (hot and cold) in higher layers.

- Maltby et al. (1986): Came up with an improvement of the Avrett (1981) model in the deep layers. These consist of a set of three models (each for a different phase of the solar cycle). Modifications of the models due to Maltby et al. (1986) have been proposed by Caccin, Gomez, and Severino (1993), Severino, Gomez, and Caccin (1994), and Ayres (1996), including constraints from more spectral lines.

- Collados et al. (1994): Presented models of the umbra of large and small spots from the inversions of Stokes $I$ and $V$ spectra from the darkest cores of three different sunspots. The variation with optical depth of temperature, magnetic-field vector, and velocity along the line of sight were obtained. Their observations confirmed that there are noticeable differences in the temperature and field strength between the umbra of large and small spots.

The temperature stratifications of all of the above-mentioned umbral models reveal an umbra that is cooler than the surrounding field-free regions in the photospheric layers, but slightly hotter in the chromosphere, while the transition to coronal temperatures takes place at a lower height in the umbra (Staude, 1986; Solanki, 1990; Collados et al., 1994).

\subsubsection{Penumbral Models and Spatially Resolved Models}

Below is a brief summary of some semi-empirical, average penumbral models and models obtained from the inversion of high-resolution polarized spectra including complete sunspots or their parts.

- Makita and Morimoto (1960), Kjeldseth Moe and Maltby (1969), Yun, Beebe, and Baggett (1984), and Ding and Fang (1989): Produced one-component models, with the latter two being primarily aimed at modeling the average penumbral chromosphere. Kjeldseth Moe and Maltby (1974) produced a two-component model of a similar kind that provided temperature as a function of optical depth in dark and bright penumbral filaments.

- del Toro Iniesta, Tarbell, and Ruiz Cobo (1994): Applied an inversion technique to a series of high-resolution filtergrams, scanning a magnetically insensitive Fe I line to study the vertical temperature and velocity structure over a small penumbral region. They provided a mean penumbral model atmosphere as a function of optical depth. 
- Westendorp Plaza et al. (2001): Besides deriving the velocity and temperature profiles, they also derived the magnetic-field stratification of a sunspot from inversions of observations of magnetically sensitive Fe I lines at $630 \mathrm{~nm}$ with a spatial resolution of approximately $1^{\prime \prime}$ and one magnetic component together with a stray-light component in each observed pixel. Similar models were provided by Mathew et al. (2003, 2004) from the inversion of infrared Fe I spectral lines at $1.56 \mu \mathrm{m}$. Mathew et al. (2004) also calculated maps of the Wilson depression and plasma parameter $\beta$.

- Rouppe van der Voort (2002): The observed radiation temperatures in the Ca II K wing were used to derive the temperature stratification of fine-structure elements in the penumbra. Three atmospheric models were constructed to represent cool, intermediate, and hot features within the penumbra, with temperature differences of order $300 \mathrm{~K}$ between them.

- Bellot Rubio (2003) and Borrero et al. (2004, 2005, 2006): Inverted the polarization profiles from the penumbra and considered the uncombed penumbral model with two magnetic components, where the component with a more horizontal and weaker field harbors the Evershed flow.

- Socas-Navarro (2005): Produced a 3D sunspot model, up to chromospheric heights, from NLTE inversion of the Ca II infrared triplet polarized spectra.

- Puschmann, Ruiz Cobo, and Martínez Pillet (2008): By assuming that the fine structure of the penumbra is spatially resolved in high-resolution Hinode/SOT data, they invert the data with a single-component model. The advantage of their modeling is a very detailed and self-consistent translation from optical depth to geometrical height scale taking into account terms of the Lorentz force and using a generic algorithm. The Wilson depression was derived this way and the model was checked to be divergence-free and in equilibrium in the horizontal and vertical directions.

Unlike quiet-Sun models, sunspot models should not be used indiscriminately in all cases and can only be taken as representative examples. As was discussed in Section 2.3, a number of observations have shown that the thermal stratification of sunspots depends sensitively on its magnetic-field strength, sunspot size, and (possibly) on the solar cycle as well.

\subsection{Magneto-Hydrostatic (MHS) Models}

The simplest static models of a pore or a sunspot ignore azimuthal variations and treat it as an axisymmetric, poloidal magnetic field (with no azimuthal component) confined to a homogeneous flux tube of circular cross-section. The equation (in cgs units) describing the MHS equilibrium of the flux tube is

$$
-\nabla p+\rho \mathbf{g}+\frac{1}{c}(\mathbf{J} \times \mathbf{B})=0
$$

where $p$ is the gas pressure, $\rho$ is the gas density, $\mathbf{g}$ is the acceleration due to gravity, $c$ is the speed of light, and $\mathbf{B}$ is the magnetic-field vector. The electric current density is given by Ampère's Law:

$$
\mathbf{J}=\frac{c}{4 \pi}(\nabla \times \mathbf{B}) .
$$

In reality, magnetic-flux concentrations lack symmetry (like most sunspots). However, occasional long-lived spots, sufficiently separated from other large flux concentrations, tend to be round and to have regular, ring-like penumbras. Therefore, an isolated, axially symmetric spot with a unipolar magnetic field is somewhat justified. Furthermore, the lifetime of sunspots is much longer than any dynamical timescale in the solar photosphere. Some 
sunspots persist for several solar rotations, essentially unchanged, whereas perturbations that propagate with the Alfvén speed would need about an hour to cross a large sunspot at its photosphere.

There are also a number of other assumptions/generalizations to keep in mind when considering MHS models: $i$ ) the MHS models are generally limited to two dimensions, the vertical and radial directions; ii) dynamic phenomena (important for shaping small-scale magnetic and thermal structure) and all fluctuations related to convective motions are usually ignored; iii) most MHS models (unrealistically) tend to treat the force-balance in isolation of the energy balance; $i v$ ) unless the energy equation is solved together with the forcebalance equation, either the magnetic field, temperature, or gas pressure must be specified, as well as an equation of state; and finally $v$ ) for models in which the force balance and energy equations are consistently solved throughout the sunspot, the convective transport is treated by applying the mixing-length formalism and the radiative transport by the diffusion approximation (see e.g. Chitre, 1963; Deinzer, 1965; Chitre and Shaviv, 1967; Yun, 1970; Jahn, 1989).

\subsubsection{Force-Free and Potential Field Models}

These are the simplest static models where the magnetic field within the sunspot flux tube is assumed to be force free, resulting in the atmosphere being horizontally stratified with variations only in the vertical direction. A number of conditions must be satisfied in order to create such a model: $i$ ) the force-free field must satisfy the condition:

$$
(\nabla \times \mathbf{B}) \times \mathbf{B}=0
$$

and $i$ ) the assumed axial-symmetry also requires that the field satisfy the current-free condition, $\nabla \times \mathbf{B}=0$, and hence $\mathbf{B}$ is a potential field $\mathbf{B}=-\nabla \phi$ (where $\phi$ satisfies Laplace's equation, $\nabla^{2} \phi=0$ ).

Potential-field models can thus be effortlessly derived by taking solutions of Laplace's equations (e.g. using dipole or Bessel function potentials). Simple models of pores were constructed Simon and Weiss (1970), using Bessel-function solutions of Laplace's equation. Subsequent advances of this model were made by Spruit (1976) and by Simon, Weiss, and Nye (1983), who represented the field in a pore by a potential field such that, at the photosphere, $B_{z}$ was uniform over a disc with a prescribed radius and zero outside it.

One obvious advantage of this method is that direct solution of Equation (1) is avoided, however the solution now involves the solution of a non-linear boundary-value and freesurface problem (i.e., determining the location of the current sheet, as potential fields fill the whole atmosphere unless bounded by a current sheet), which is nontrivial.

\subsubsection{Current-Sheet Models}

A more satisfactory potential-field model can be derived by constructing a field contained within a flux tube such that the difference between the internal $\left[p_{\mathrm{i}}\right]$ and external $\left[p_{\mathrm{e}}\right]$ gas pressures is balanced by the Lorentz force in a thin current sheet bounding the flux tube, such that

$$
p_{\mathrm{i}}+\frac{B^{2}}{8 \pi}=p_{\mathrm{e}}
$$

This bounding current sheet is referred to as the "magnetopause". 
Approximate solutions have been found and applied to sunspots and pores by Simon and Weiss (1970) and Simon, Weiss, and Nye (1983). Wegmann (1981) produced the first general solution to the free-boundary problem and Schmidt and Wegmann (1983) were the first to apply this technique to sunspots, successfully modeling pores. More advanced currentsheet models have succeeded in producing relatively realistic sunspot models, including the penumbra. These models are briefly summarized below.

- Jahn (1989): Provided an extension of the Schmidt and Wegmann (1983) model by introducing body/volume currents (distributed in the outer parts of a flux tube, below the photosphere of the penumbra), in addition to a current sheet at the sunspot-quiet-Sun boundary. The body currents contribute to the lateral force balance and affect the pressure stratification, so that the gas in the penumbra is hotter, thus layers of equal gas pressure assume higher levels than in an umbra where the field is current free. A fit to the magnetic and photometric profiles (taken from Beckers and Schröter, 1969) provided a distribution of the electric currents in their model.

- Jahn and Schmidt (1994): Introduced two current sheets, one at the outer boundary of the flux tube (the magnetopause) and the other at the interface between the penumbra and the umbra. They did this in order to obtain a more realistic thermal structure of the sunspot with distinctively different umbral and penumbral thermal mechanisms. They also assumed that the umbra is thermally insulated from the penumbra, while some of the energy radiated from the penumbra itself is supplied by convective processes that transfer energy across the magnetopause.

- Pizzo (1990): Extended the earlier work of Pizzo (1986) (see Section 4.2.4), using multigrid techniques to calculate the magnetic structure of a sunspot bounded by a current sheet.

In general, current-sheet models are consistent with the concept that sunspots represent discrete, erupted, magnetic entities (Solanki, 2003). Observational support for the currentsheet description of spots is taken from the sharp transition between the umbra and quiet photosphere in pores and from the relatively uniform photometric appearance of most umbrae (Gokhale and Zwaan, 1972). The fact that the magnetic field is so large at the whitelight boundary of the sunspot also strongly suggests that sunspots are bounded by a current sheet (Solanki and Schmidt, 1993). However, as Solanki (2003) points out, the rugged nature of the sunspot boundary in white-light images means that the current sheet is not as well defined as one might picture on the basis of simple flux-tube models. Further evidence for a current sheet surrounding sunspots comes from observations suggesting that the field inside sunspots is close to potential. This suggests that the currents bounding the strong field must be mainly located in a relatively thin sheet at the magnetopause (see e.g. Lites and Skumanich, 1990).

\subsubsection{Self-Similar Fields}

There is considerable arbitrariness in assigning the distribution of the volume current and the corresponding radial structure in the sunspot atmosphere. As first shown by Schlüter and Temesváry (1958) (and later extended by others, e.g. Chitre, 1963; Jakimiec, 1965; Jakimiec and Zabża, 1966; Chitre and Shaviv, 1967), the problem can be greatly simplified by assuming a self-similar profile for the magnetic field.

In cylindrical coordinates, the (untwisted) $B_{z}$ and $B_{r}$ components of the magnetic field take the form

$$
B_{z}(r, z)=f(\zeta) B_{0}(z),
$$




$$
B_{r}(r, z)=-\frac{r}{2} f(\zeta) \frac{\mathrm{d} B_{0}(z)}{\mathrm{d} z}
$$

where $r$ and $z$ refer to the radial and vertical coordinates respectively, $B_{0}(z)$ is the field strength at the flux-tube axis, and $\zeta=r \sqrt{B_{0}(z)}$. The shape of the function $f(\zeta)$ may be freely chosen (usually a Gaussian). For a non-constant $f(\zeta)$, inserting Equations (5) and (6) into (1) reduces the equation of MHS equilibrium to the following equations:

$$
\begin{aligned}
& 0=-\frac{\partial p}{\partial r}+\frac{B_{z}}{4 \pi}\left(\frac{\partial B_{r}}{\partial z}-\frac{\partial B_{z}}{\partial r}\right), \\
& 0=-\frac{\partial p}{\partial z}-\frac{B_{r}}{4 \pi}\left(\frac{\partial B_{r}}{\partial z}-\frac{\partial B_{z}}{\partial r}\right)-\rho g .
\end{aligned}
$$

Integrating Equation (7) over $r$ from 0 to infinity for constant $z$ leads to the following expression for $\Delta p(z)=p_{\mathrm{e}}(z)-p_{\mathrm{i}}(z)$, the difference in gas pressure between the external $[p(\infty, z)]$ and internal $[p(0, z)]$ regions:

$$
\Delta p(z)=-\frac{1}{8 \pi}\left(\frac{\Phi}{2 \pi} y \frac{\mathrm{d}^{2} y}{\mathrm{~d} z^{2}}-y^{4}\right),
$$

where $\Phi$ denotes the total magnetic flux and $y=\sqrt{B_{0}(z)}$. Thus this method has the advantage that it simplifies the mathematical treatment of MHS equilibrium by reducing the partial differential equation to a second-order, ordinary differential equation for the field strength at the axis of the spot by specifying the cross-sectional shape of the magnetic-field distribution within an axisymmetric flux tube.

A general assumption made is that the distribution of magnetic flux on horizontal planes is geometrically similar at each depth. Furthermore, in contrast to current-sheet models, self-similarity allows for a continuous variation of field strength and gas pressure across the spot. The field falls off smoothly from the central axis value to zero at large radial distances (hence, there is no clear definable "inside" or "outside" of the spot in this description, therefore essentially ignoring the fact that sunspots have sharp edges). Thus, there is the computational convenience as treatment of the discontinuity associated with a current sheet is avoided. However, the similarity law enforces a somewhat arbitrary distribution of electric current, resulting in the appearance of a bright ring in the emergent intensity. Those currents determine (to some extent) the horizontal temperature variations, which in general need not comply with the observed photometric profile (Jahn, 1992).

A further disadvantage of a purely self-similar sunspot model is that negative pressures and densities are often obtained in the photospheric and upper atmospheric layers of the flux tube, due to the fact that the hydrodynamic pressure and density are decreasing exponentially with height, while the magnetic field does not quite decrease at the same rate. There have been some proposed work-arounds to this problem (e.g. Hanasoge, 2008), however, such methods are not ideal as they tend to substantially alter the governing differential equations because they require the inclusion of terms in the ideal MHD equations which are not physical.

Furthermore, since the Lorentz force also drops with height, the magnetic field essentially becomes an unbounded, force-free field within a few pressure scale heights above the photosphere, resulting in a field configuration not too different from a potential field. This fact implies that self-similar models are essentially not force free in the regions where they should be. Since the shape of the magnetic field at the surface is sensitive to this, one has 
here a model that breaks down in an essential way in just those regions where diagnostics are best.

Nonetheless, due to their simplicity, similarity expansions have been utilized to generate the field configuration for a number of studies, some of which are summarized below.

- Deinzer (1965): Generalized the Schlüter and Temesváry (1958) model, where the similarity law for the magnetic field is coupled with the thermodynamic structure along the axis of a spot, as described by mixing-length theory.

- Yun (1970): Improved the Deinzer (1965) model by the introduction of an "effective surface monopole", which controlled the inclination of the particular field lines identified with the outer edge a spot at the surface. Hence, the upper boundary condition takes into account the fact that the gas pressure difference at the photospheric level is not negligible (as assumed by Deinzer, 1965). Lower boundary conditions were also modified, as the effects of partial ionization on the relation between the internal and external pressures and temperatures were included.

- Yun (1971) and Osherovich and Flaa (1983): Demonstrated that the introduction of a moderately twisted field $\left(\approx 17^{\circ}\right.$ near the surface, compatible with observations) contributes little to the force balance in spots and changes only slightly the main characteristics of the model (as already mentioned in Section 2.2.2).

- Landman and Finn (1979): Imposed an Evershed-type radial velocity distribution in the upper region of the spot atmosphere, in order to get a satisfactory continuum-intensity profile across the sunspot. However, relatively large values of the Evershed flow (i.e., close to $10 \mathrm{~km} \mathrm{~s}^{-1}$ ) were required to obtain a satisfactory temperature profile.

- Low (1980): Prescribed a method for generating exact solutions of MHS equilibrium describing a cylindrically symmetric magnetic flux tube oriented vertically in a stratified medium. Given the geometric shape of the field lines, compact formulae were presented for the direct calculation of all the possible distributions of pressure, density, temperature, and magnetic-field strength compatible with these field lines under the condition of static equilibrium. A particular solution was obtained by this method for a medium-sized sunspot whose magnetic field obeys the similarity law of Schlüter and Temesváry (1958).

- Osherovich (1982): Extended self-similar models to include field lines in the outer part of the sunspot that return to the solar surface just outside the visible sunspot (return flux). The emerging flux constitutes the penumbra. The predicted continuum intensity of returnflux models was not much closer to the observations than the standard self-similar models.

- Fla, Skumanich, and Osherovich (1982): Applied the return-flux model to a spot with the observational data of Lites and Skumanich (1982) for pressure, maximum-field strength, and size. The force-balance equation was solved to obtain self-consistent magnetic field, pressure, and temperature distributions. The resulting distributions appeared to yield improved representations of umbral - penumbra and penumbra - quiet-Sun boundaries compared to regular (e.g. Schlüter and Temesváry, 1958) self-similar models. However, it appears that one needs to introduce an Evershed flow to eliminate the apparent umbral bright ring in the continuum. Similar work on return-flux sunspot models was undertaken by Osherovich and Lawrence (1983), Osherovich and Garcia (1989), and Liu and Song (1996).

- Solov'ev (1997): Extended the self-similar sunspot models by introducing a current sheet at the sunspot boundary.

- Moon, Yun, and Park (1998): Included a description of the energy balance and the observed horizontal variation of the Wilson depression when determining the shape function from the observed radial dependence of the magnetic field. 
- Cameron, Gizon, and Duvall (2008), Hanasoge (2008), Moradi, Hanasoge, and Cally (2009), and Shelyag et al. (2009): All employed simple self-similar toy sunspot models in conducting numerical simulations of helioseismic wave propagation through magnetized plasmas.

As we shall see in the following sections however, constructing more physically realistic sunspot models is practical, especially with the MHD codes (Section 6) that are currently used for the wave-propagation problem.

\subsubsection{Solution of Full MHS Force Balance}

These methods involve solving for the magnetic field on the basis of full MHD equilibrium, with and without a current sheet. Pressure is specified throughout the numerical domain (usually as a function of depth and taken from semi-empirical models), partly depending on the distribution of field lines (hydrostatic equilibrium acts along each field line).

Pizzo (1986) utilizes the description of Low (1975), who proposed transforming the thermodynamic parameters (the pressure and temperature of the gas) into functions of the magnetic vector potential and depth. In this form, a functional form is prescribed for the gas pressure, but not for the magnetic field as in the similarity models, and the equilibrium is solved as a classical non-linear boundary-value problem.

By transforming the pressure and density into functions of the field-line constant $u$ (used by both Low, 1980 and Pizzo, 1986, it essentially determines the shape of the field lines) and height $z$, and requiring hydrostatic equilibrium along the field lines, Equation (1) can be reduced to a single scalar equation describing the magnetostatic equilibrium of an axisymmetric, poloidal field:

$$
\frac{\partial^{2} u}{\partial r^{2}}-\frac{1}{r} \frac{\partial u}{\partial r}+\frac{\partial^{2} u}{\partial z^{2}}=-4 \pi r^{2} \frac{\partial P(u, z)}{\partial u},
$$

where $P$ is a function related to the gas-pressure distribution. Low (1975) provides an approximation for the distribution of gas pressure along the magnetic-field lines in a vertical, axisymmetric flux tube in magnetostatic equilibrium,

$$
P(u, z)=P_{0}(u) \exp \left[-\int_{0}^{z} \frac{\mathrm{d} z^{\prime}}{h\left(u, z^{\prime}\right)}\right],
$$

where $P_{0}(u)$ is the gas pressure along the lower boundary, $h(u, z)$ is the isothermal scale height $[h=\mathcal{R} T / \mu g$, where $\mathcal{R}$ denotes the ideal gas constant, $T$ the temperature, $\mu$ the mean molecular weight, and $g$ is the acceleration due to gravity] for a plasma obeying the ideal gas law $[p=\rho \mathcal{R} T / \mu]$. The $u=$ constant curves describe the field lines of the system. The vertical and radial field components may then be expressed in terms of $u$ :

$$
B_{z}=\frac{1}{r} \frac{\partial u}{\partial r}
$$

and

$$
B_{r}=-\frac{1}{r} \frac{\partial u}{\partial z} .
$$

The range of validity is determined by the representative pressure distributions along the axis and in the field-free atmosphere. 
The gas-pressure difference between the quiet photosphere and the axis of the spot is needed for the computation. Pizzo (1986) takes these values from the semi-empirical models of the umbral photosphere derived by Avrett (1981). Pizzo (1986) then develops a method for the iterative numerical solution of Equation (10), essentially a second-order, non-linear, elliptic, partial differential equation, which can be easily solved using standard numerical techniques in the case of fixed boundary conditions.

An advantage of this model is that the Wilson depression and net internal - external pressure difference can be adjusted by vertical translation of the absolute height scales of the two reference atmospheres. However, the configuration considered by Pizzo (1986) has its base placed $120 \mathrm{~km}$ below the visible surface of the umbra, which corresponds to $z=0$ in the Avrett (1981) model, hence his models do not address the question of the spot structure in deeper layers. The model also assumes a Gaussian profile of the magnetic field across the base (i.e. self-similar), thus ignoring the existence of a discontinuous transition from the magnetized to field-free plasma (i.e., a current sheet). However, Pizzo (1990) later extends his method by incorporating the free-surface problem in the solution of the equation of magnetostatic equilibrium for a flux tube surrounded by an infinitely thin current sheet, utilizing a body-fitted mesh generation and multi-grid relaxation techniques for solving Equation (10).

Steiner, Pneuman, and Stenflo (1986) also developed a method for the iterative numerical solution of Equation (10), including a boundary current sheet and also field twist in their treatment, while Cally (1991) adopted a full multi-grid method to tackle the free-boundary problem by formulating it in terms of inverse or flux coordinates, in which the magneticfield lines become coordinate lines. This results in the energy equation reducing to ordinary differential equations along field lines when the radiation is optically thin. Also, if steady plasma flow is allowed, Alfvén's theorem guarantees that there can be no cross-field component of velocity, i.e. that the fluid flows along field lines.

Khomenko and Collados (2006) used the Pizzo (1986) sunspot model in numerical simulations of magnetoacoustic wave propagation, and more recently Khomenko and Collados (2008) produced a new set of models consisting of concatenation of self-similar models in the deep layers, where the gas pressure dominates over magnetic pressure, with models in which the pressure distribution is prescribed on the axis. In the deep photospheric layers, a self-similar solution for the magnetic field is calculated following the method of Low (1980), while the pressure and density distributions with height and radius are found from analytical expressions. A potential solution is then generated above some arbitrary height using the method of Pizzo (1986) - the bottom boundary of this model coincides with the top boundary of the deep photospheric/self-similar model (approximately $z=-1 \mathrm{Mm}$; it can be adjusted, however, to limit the upper boundary of the self-similar model). This solution is then used as an initial guess in the integration of the complete force balance equation along the magnetic-field lines, i.e. Equation (10).

The analytical description of the pressure distribution along magnetic-field lines is taken from Pizzo (1986) and Low (1975). The Model S (Christensen-Dalsgaard et al., 1996) pressure distribution for the field-free atmosphere is smoothly joined to the VAL-C (Vernazza, Avrett, and Loeser, 1981) model of the solar chromosphere. On the axis, the Avrett (1981) umbral-core model is used in the upper layers, while the linear inversion model of Kosovichev, Duvall, and Scherrer (2000) is used for deeper layers (down to a depth of $1 \mathrm{Mm}$, thus ignoring the "hot" layer), which takes the Wilson depression to be at $450 \mathrm{~km}$. A smooth transition between the models is then calculated for the gas pressure and scale-height distributions, in the same manner as Pizzo (1986), and by changing the parameters of the solution, a set of models with the desired properties can be produced. However, as with the Pizzo 
(1986) and all other pressure-distributed sunspot models, the vertical extent of these models is severely limited by available semi-empirical data on the sunspot axis.

\subsubsection{Extrapolated Field Models}

Martens et al. (1996) present a force-free, constant- $\alpha$ model for the magnetic field in and above a fluted sunspot. They demonstrate that magnetograms for round sunspots can essentially be matched by a series of Bessel functions. Their model parameters are chosen to reproduce the high-resolution observations (magnetograms) of Title et al. (1993) at the 1-m Swedish Solar Observatory at La Palma, and an analytical expression is obtained for the 3D magnetic field emanating from the sunspot's umbra and penumbra. The model accurately reproduces the azimuthal variation in inclination angle, as well as the mean constancy of the magnetic-field strength, and the appearance of a highly corrugated neutral line on the limb side of off-center sunspots.

Another model based on matching magnetograms with analytical expressions is the axisymmetric sunspot model of Moradi and Cally (2008), which consists of a non-potential, untwisted, 3D MHS sunspot model constrained to fit observed surface magnetic-field profiles. The preferred surface-field configuration of the sunspot model was derived from constrained polynomial fits to the observed scatter plots of the radial $\left[B_{r}\right]$ and vertical $\left[B_{z}\right]$ surface magnetic-field profiles of AR 9026 on 5 June 2000 - a fairly symmetrical sunspot near disk center, ideal for helioseismic analysis - obtained from IVM (Imaging Vector Magnetograph) vector magnetograms. The surface field is therefore quite realistic, which is important because there is evidence (e.g. Schunker and Cally, 2006) that magnetic effects in helioseismology are dominated by the top few hundred kilometers. The fits of $B_{r}$ and $B_{z}$ are then used to derive an analytical form for the flux function. Instead of a current sheet along the boundary, the authors formulate an analytical form for the outermost field line to allow field strength to smoothly drop to zero. However, in a similar vein to other sunspot models that derive their thermodynamic properties solely from a prescribed magnetic-field configuration (e.g. self-similar models, Section 4.2.3), the axial pressure and density of these models posses a high degree of sensitivity near the upper boundary, with a tendency to become negative in the photospheric layers and beyond.

\subsection{Non-MHS Models}

\subsubsection{Semi-Empirical Sunspot Models}

Very close to the solar surface, the properties of sunspots can be inferred from spectropolarimetric measurements. These layers are the most important to model since it is exactly in this region that the effects on the wave cannot be treated assuming only weak perturbations. Cameron et al. (2010) have emphasized the desirability of incorporating this information into helioseismic wave-propagation simulations by constructing simplified, axisymmetric sunspot models that are designed to capture the effects of the first few hundred $\mathrm{km}$.

Since full spectro-polarimetric inversions are available for very few spots (e.g. Mathew et al., 2004), Cameron et al. (2010) utilize a combination of existing semi-empirical models: the umbral model of Maltby et al. (1986) and the penumbral model of Ding and Fang (1989), which cover the range of heights from about $500 \mathrm{~km}$ below the quietSun $\tau=1$ level to the lower chromosphere. The OPAL equation of state tables are used to infer sound speed, temperature, pressure, and density profiles. Since the aim is 

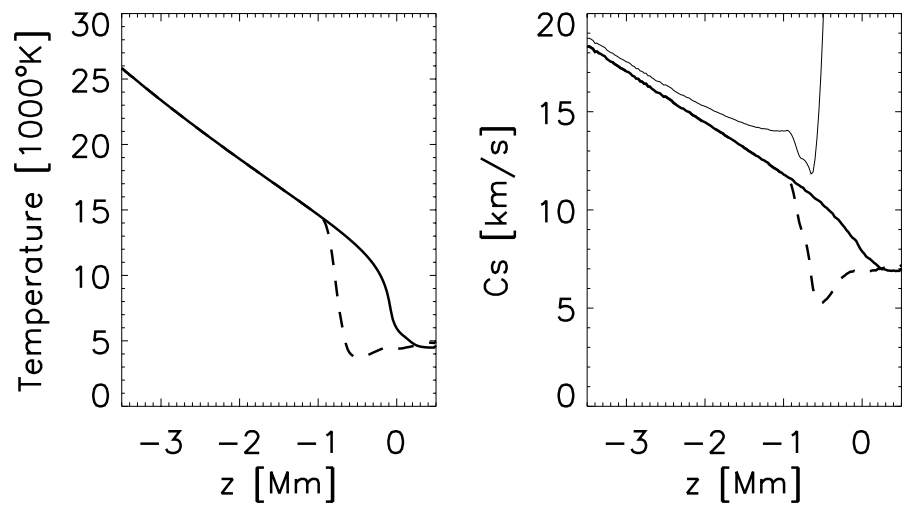

Figure 3 Thermodynamic, sound and fast mode speed profiles of the sunspot model of AR 9787 produced by Cameron et al. (2010). Left panel: Vertical temperature profile of reference stratification (solid) and near the center of the umbra (dashed). Right panel: Vertical sound-speed profile of reference stratification (solid) and near the center of the umbra (dashed). The dotted line shows the fast-mode speed in the center of the umbra.

only to model the near-surface region, the atmospheric models are smoothly matched to the Model S (Christensen-Dalsgaard et al., 1996) quiet-Sun atmosphere below $\tau_{5000}=0$ and $400 \mathrm{~km}$ above. Cameron et al. (2010) combine these 1D models to form a 3D, axisymmetric model of the sunspot in AR 9787, with the umbral and penumbral radii chosen to match those of the observed sunspot (10 and $20 \mathrm{Mm}$, respectively). The model is then stabilized with respect to convection by calculating the relative perturbations with respect to the quiet-Sun atmosphere and imposing these on the stabilized model (see Section 6.2 for details). As good measurements of the magnetic field are unavailable for the sunspot in AR 9787, the model assumes that $B_{z}$ is described by a Gaussian horizontal profile and the vertical profile is tuned so the field inclination at the umbra-penumbra border is approximately $45^{\circ}$. Figure 3 shows the vertical temperature, sound and fast mode speed profiles in the center of the umbra of the sunspot model.

\subsubsection{Dynamical Models}

Kitchatinov and Rüdiger (2007) propose a theoretical sunspot model, with complete dynamics of both magnetic field and flow, which can reproduce the observed bright rings around sunspots (e.g. Rast et al., 1999, 2001). A simplified model was initially used to probe the possibility of reproducing bright rings by heat transport alone. In this model the mean flow velocity is put to zero, the spot-like structure of the magnetic field is prescribed and stationary, and the diffusion equation for the entropy is solved. The authors find that the brightness of the region occupied by magnetic field decreases due to magnetic quenching of the thermal diffusivity; however, the resulting surface brightness profile did not show a bright ring.

A more consistent MHD model of a sunspot was then considered using the complete momentum equation together with the induction equation. Amplitudes of the initial uniform field of several hundred Gauss were considered. The surface flow generated is convergent near the spot but divergent at larger distances. The amplitude of the flow modeled was approximately $800 \mathrm{~m} \mathrm{~s}^{-1}$. Both brightness and field strength (central value of about $2700 \mathrm{G}$ ) 
were modeled to be almost uniform in the central parts of the sunspot and changing rapidly with radius beyond. The radial heat-flux profiles from their simulations show consistent bright rings around the spots, appearing to be somewhat brighter than the observed rings. To probe the contribution of the flow to the bright rings, the field is switched off. The bright rings do not disappear, which leads the authors to conclude that both the flow and the reduced diffusivity quenching contribute to the resulting bright rings.

Another set of dynamical MHD sunspot models, consisting of idealized axisymmetric flux tubes (where the magnetic field is matched to a potential field at the upper boundary) in a compressible convecting atmosphere, were presented by Hurlburt and Rucklidge (2000). In their models, they find the magnetic flux to be confined by an inward "collar" flow at the surface. Further outside, the flow direction appears to reverse and a moat cell appears. The authors suggest that the collar cell holding sunspots together is hidden beneath their penumbrae, so that only the outflow in the moat cell is visible at the surface. Although this particular flow pattern (i.e., inflows and downflows around sunspots, first proposed by Parker, 1979), can in theory help to explain the question of the stability of long-lived sunspots, there are a number of theoretical concerns with this conjecture (already discussed in Section 3). Furthermore, the existence of such a flow structure around sunspots is absent from both observations and recent helioseismic inferences (e.g. Section 7.6.2), as well as realistic radiative MHD simulations of sunspot structure (Heinemann et al., 2007; Rempel, Schüssler, and Knölker, 2009a; Rempel et al., 2009b; see below for details).

\subsection{Numerical Simulations of Radiative Magnetoconvection}

Significant progress in our ability to simulate sunspots using realistic MHD simulations (i.e. MHD simulations that include the solar equation of state and multi-dimensional radiative transfer) was only possible during the past couple of years. This is primarily due to the fact that pursuing radiative MHD simulations on the scale of sunspots with sufficient resolution for capturing the essential scales of magneto-convective energy transport requires fairly large computational domains and accordingly computing power. In addition to the computational domain size the physical parameters encountered in and above the umbral region of a sunspot pose significant numerical challenges. The combination of several $\mathrm{kG}$ for the magnetic field with the rather small density scale height leads to a steep increase of the Alfvén velocity above the sunspot umbra, reaching values in excess of a few $1000 \mathrm{~km} \mathrm{~s}^{-1}$. Such high velocities lead to severe time-step constraints for explicit codes that make such a simulation almost impractical, unless this constraint is relaxed by artificially limiting the Lorentz force in low $\beta$ regions.

3D models with a slender, rectangular, slab geometry have been used to study the inner penumbra (Heinemann et al., 2007; Rempel, Schüssler, and Knölker, 2009a). The results from the MHD simulations show the formation of filamentary structures resembling those in the inner penumbra of a real sunspot, including bright filaments containing central dark cores. More recent 3D calculations of round sunspots, and even pairs of opposite polarity spots (e.g. Rempel et al., 2009b), also show extended outer penumbrae with realistic Evershed flows, having mean flow velocities of up to $6 \mathrm{~km} \mathrm{~s}^{-1}$.

Most of these simulations are focused around the sunspot fine structure (umbral dots and penumbral filaments) and cover only a temporal evolution of a few hours. Simulations addressing the long-term evolution of sunspots (on a time scale of days) are currently only feasible if the resolution is decreased, therefore not resolving details of the fine structure as well as penumbral regions. Figure 4 displays the bolometric intensity and subsurface magnetic-field strength for a sunspot evolved over a total of 15 hours. Figure 5 compares 
Figure 4 Simulated sunspot in a $50 \times 50 \times 8 \mathrm{Mm}$ domain. The top panel displays the bolometric intensity, the bottom panel field strength on a cut through the center of the spot.
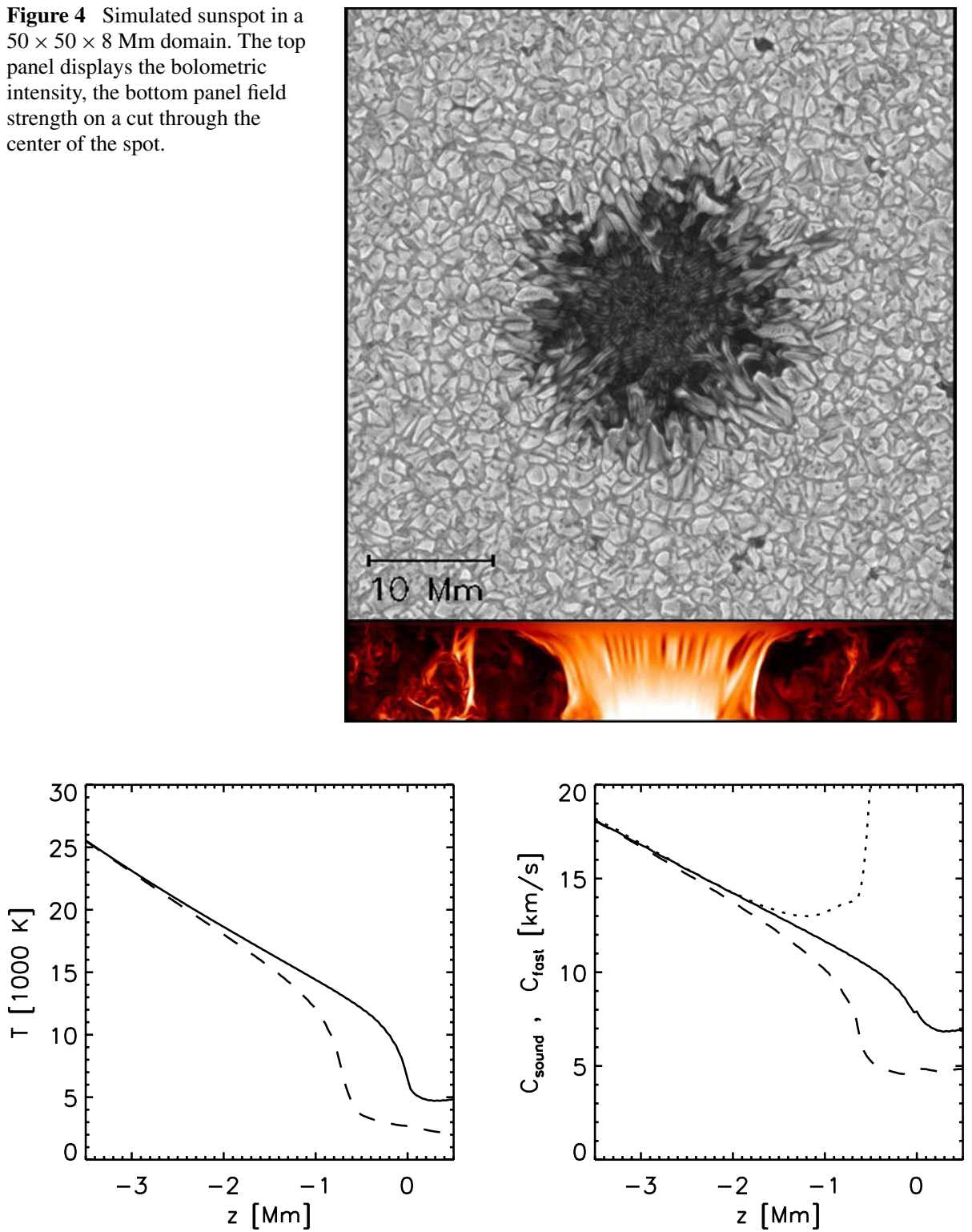

Figure 5 Left panel: Vertical temperature profile of the reference stratification (solid) and near the center of the umbra (dashed) of the sunspot model in Figure 4. The large temperature perturbation corresponds to a Wilson depression of about $600 \mathrm{~km}$. Right panel: Vertical sound-speed profile of reference stratification (solid) and near the center of the umbra (dashed). The dotted line shows the fast-mode speed in the center of the umbra.

the profiles of temperature, sound speed, and fast mode speed in the center of the spot to those of the reference stratification near the edges of the domain. The temperature profile (left panel) shows a Wilson depression of about $600 \mathrm{~km}$ which forms rather quickly during the first few hours of the simulation, and deeper down a slowly progressing cooling front 
leads to a moderate adjustment of temperature in layers $1-2 \mathrm{Mm}$ beneath the $\tau=1$ level in the umbra corresponding to changes of the speed of sound (right panel) on the order of a few $100 \mathrm{~m} \mathrm{~s}^{-1}$ to about $1 \mathrm{~km} \mathrm{~s}^{-1}$. The fast-mode speed shows a steep increase above the $\tau=1$ level in the umbra, the maximum speed is here artificially limited to about $60 \mathrm{~km} \mathrm{~s}^{-1}$ to relax the stringent Alfvénic time-step constraint.

While these simulations provide a realistic description of the thermal and magnetic structure in the uppermost few Mm of a sunspot, they cannot address fundamental questions regarding the subsurface structure and origin of sunspots. Currently the domains are with 8 $\mathrm{Mm}$ still rather shallow (deeper domains up to $16 \mathrm{Mm}$ are in preparation) and the initial field structure is based on a monolithic model. Furthermore the magnetic field is fixed at the bottom boundary. Nevertheless these models provide very useful artificial oscillation data that can be used to test helioseismic inversion methods, since the latter requires primarily a consistent, rather than a fully realistic model.

\section{Diagnostics Potential of Helioseismology}

In the previous section we reviewed models of sunspots; each of these provides a prescription for computing the subsurface structure (i.e. the 3D forms of the sound speed, density, and magnetic field) of a model sunspot over some range of depths. These prescriptions are based on a wide variety of physical assumptions. In general, it is not known which of these assumptions are good approximations (in the sense of accurately describing solar conditions). Helioseismology promises the ability to infer the subsurface structure of sunspots and active regions, and thus to test the physical approximations that are used in building models of these regions.

Helioseismology has shown (see Gizon and Birch, 2005 for a review) that wave propagation is different in sunspots in the quiet Sun. For example, single-bounce wave travel times are altered by (order of magnitude) about thirty seconds; the details depend strongly on the data-analysis filters, the first-bounce distance, and the wave frequency (e.g. Braun and Birch, 2008). In Section 7 we provide a follow-up to the detailed helioseismic study undertaken by Gizon et al. (2009a, 2009b) of the active region AR 9787. These measurements clearly demonstrate that helioseismology can measure the changes in wave propagation that are associated with sunspots and active regions.

Many conclutions have been drawn about sunspot and active-region structure from local helioseismology (e.g. Fan, Braun, and Chou, 1995; Kosovichev, 1996; Jensen, Jacobsen, and Christensen-Dalsgaard, 1998; Chou, Sun, and Chang, 2000; Kosovichev, Duvall, and Scherrer, 2000; Zhao and Kosovichev, 2003; Couvidat et al., 2004; Basu, Antia, and Bogart, 2004; Crouch et al., 2005; Bogart et al., 2008). Despite the long history of work on this topic, there is no general agreement on the subsurface structure of active regions. Here we will investigate some of the possible causes of the disagreement shown by Gizon et al. (2009a, 2009b): the frequency dependence of travel times (Section 7.2), the effects of phase-speed filters on measurements (Section 7.3), and the effects of inversion parameter choices and inclusion of surface terms on ring-diagram structure inversions (Section 7.4).

Another approach to using helioseismology to constrain sunspot models is the direct simulation of wave propagation through model sunspots and comparison of the resulting wave field with the helioseismic measurements (e.g. Cameron, Gizon, and Daiffallah, 2007; Cameron, Gizon, and Duvall, 2008; Moradi, Hanasoge, and Cally, 2009). This approach bypasses the need for linear forward models (i.e. models for the linear sensitivity of travel-time 
shifts or ring-diagram frequency shifts to changes in subsurface structure). The approximations made in the process of formulating linear forward models may be one source of the disagreement shown by Gizon et al. (2009a, 2009b). A limitation of the direct simulation approach is that it is extremely computationally intensive. The examples shown in Section 7.8 demonstrate that numerical simulations are capable of reproducing many features of the helioseismic measurements (see also Cameron, Gizon, and Duvall, 2008). In addition, simulations promise to provide a powerful tool for determining the sensitivity of helioseismic measurements to small changes in the subsurface structure of sunspot models. This type of study is required to be able to make meaningful estimates of the signal-to-noise ratios required for the helioseismic measurements to constrain any particular aspect of sunspot models.

\section{Numerical Forward Modeling of Waves Through Model Sunspots}

\subsection{Numerical Methods}

Our best chance at constraining the interior structure of sunspots comes with constructing accurate, numerical, forward models. There are two major reasons for claiming this:

i) The departure from the quiet-Sun wave-propagation physics being so dramatic in a sunspot, it is quite likely that the single-scattering Born approximation (e.g. Birch, Kosovichev, and Duvall, 2004) fails entirely (e.g. Gizon, Hanasoge, and Birch, 2006). It is therefore imperative to solve the wave-propagation equations in a fully $3 \mathrm{D}$, highly magnetized environment. However, the basis of semi-analytical sunspot formalisms is significantly undermined by the sheer mathematical complexity of dealing with the MHD equations (not for the want of trying; e.g. Bogdan, 1999), and hence the necessity of using purely numerical techniques.

ii) Computational solvers are flexible and can be rather easily extended to include more effects, such as flows, changes on wave sources, etc.

The difficulties in creating an MHD solver that produces sufficiently accurate results are not to be underestimated. A number of groups are working on this problem (Khomenko and Collados, 2006; Cameron, Gizon, and Daiffallah, 2007; Parchevsky and Kosovichev, 2007; Hanasoge, 2008; Shelyag et al., 2009). The numerical techniques, and validation and verification procedures vary significantly from one code to another. A further complexity is introduced by an Alfvén speed that increases exponentially with height, approaching values of several hundred $\mathrm{km} \mathrm{s}^{-1}$ near the computational upper boundary. Simulating wave propagation in a plasma so highly magnetically dominated is remarkably expensive, especially in 3D. A number of simulations (e.g. Cameron, Gizon, and Daiffallah, 2007; Cameron, Gizon, and Duvall, 2008; Hanasoge, 2008; Moradi, Hanasoge, and Cally, 2009; Rempel, Schüssler, and Knölker, 2009a) apply an Alfvén wave-speed limiter to moderate the action of the Lorentz forces, thereby indirectly controlling the plasma- $\beta$. Having described this context, three rather serious issues remain as yet outstanding: $i$ ) What are the spatiotemporal resolution requirements for simulating waves in an MHD environment? ii) How do we treat the upper magnetic boundary? and iii) What harm does the Alfvén-speed limiting term do to the near and far scattered wave field?

As yet, only parts of these questions have been answered. This may be attributed to a systemic shortsighted forward modeling approach in our intensely observationally driven field: simulate and obtain results as quickly as possible. Evidently, we must attempt to overturn this trend by $i$ ) ensuring that the numerical methods are high order 
and highly precise, $\mathrm{ii}$ ) validating and verifying that the equations are being solved accurately (i.e. testing against a number of known solutions), iii) determining and matching the spatio-temporal resolution requirements, $i v$ ) using stable, physically-motivated, welltested boundary conditions, and finally $v$ ) testing that the approximations used in the calculations do not directly affect the quality of the solution. Of course, attendant questions of computational efficiency must also be addressed, because the forward-modeling approach requires exhaustive testing of sunspot models, requiring a large number of calculations.

\subsection{Background Models Stabilized Against Convective Instability}

Numerical simulations of seismic wave propagation through random media must begin with an initial background model of the Sun. In helioseismology, it is common practice to begin with Model S (e.g. Cameron, Gizon, and Daiffallah, 2007; Parchevsky and Kosovichev, 2007; Khomenko et al., 2009). However, most background models include convection and numerical forward models which simulate linear wave propagation are sensitive to this. Therefore, it is essential to remove such convective instabilities from the background in order to be able to successfully simulate linear wave propagation on the time scalesk $(\approx$ eight hours) required for computational helioseismology, as well as to ensure that convective modes do not swamp the signatures one is interested in analyzing.

There are a number of ways to stabilize background models against convection (e.g. Hanasoge et al., 2006; Cameron, Gizon, and Daiffallah, 2007; Parchevsky and Kosovichev, 2007; Shelyag, Fedun, and Erdélyi, 2008), all requiring zero buoyancy so that a vertically displaced packet of gas in adiabatic equilibrium will not continue to rise, i.e. ensuring that the Brunt-Väisälä frequency always remains positive, specifically $N^{2} / g>0$ where $g$ is the gravitational acceleration. There are a number of ways to go about this; for example, the method employed by Parchevsky and Kosovichev (2007) ensures that, when this condition is not met, the value is replaced with zero, or very small values $\left(\right.$ e.g. $\left.3 \times 10^{-5} \mathrm{Mm}^{-1}\right)$. Shelyag, Fedun, and Erdélyi (2008) and Shelyag et al. (2009) have a slightly different approach, whereby they adjust the pressure and density of Model S using the equation of state for an ideal gas to retain a constant $\Gamma_{1}=5 / 3$, with the additional constraint that the modified sound-speed profile does not differ substantially from the original. Both Parchevsky and Kosovichev (2007) and Shelyag et al. (2009) have shown relatively solar-like power spectrums.

Another method has been employed by Cameron, Gizon, and Daiffallah (2007) and Cameron, Gizon, and Duvall (2008), and it has undergone detailed development and testing by Schunker, Cameron, and Gizon (2010), whereby the Model S background is stabilized against convective instability by ensuring $\partial_{z} p=\max \left(c^{2} \partial_{z} \rho_{0}-\epsilon, \partial_{z} p_{0}\right)$, where $\partial_{z}$ specifies the partial derivative with respect to height, the subscript " 0 " indicates unperturbed Model $S$ values, and $\epsilon=10^{-5} \mathrm{~g} \mathrm{~s}^{-2}$ near the surface and zero everywhere else. These changes to the background model alter the eigenmodes of the problem, and this must be taken into account. Schunker, Cameron, and Gizon (2010) have attempted to do this by examining the eigenmodes and ensuring that they are solar-like and have successfully demonstrated quite solar-like power spectra. Other ways to remove the convective instability have not been explored however, and it is not clear which form of stabilization least affects the eigenmodes.

\subsection{Numerical Codes}

In this section we provide a brief summary of the four numerical simulation codes that were discussed and used during the two HELAS workshops on sunspot seismology held in 2007 
and 2009. These codes compute the propagation of solar waves through prescribed background models. We note that other numerical simulation codes also exist (e.g. Parchevsky and Kosovichev, 2007; Hartlep, Miesch, and Mansour, 2008).

\subsubsection{The IAC MHD Code}

The IAC MHD code, described by Khomenko and Collados (2006) and Khomenko, Collados, and Felipe (2008), solves the non-linear MHD equations for perturbations, written in the conservative form, using a fourth-order central difference scheme and advanced in time by a fourth-order Runge - Kutta method. In a similar manner to Stein and Nordlund (1998) and Caunt and Korpi (2001), in order to damp high-frequency numerical noise on subgrid scales, the physical diffusive terms in the equations of momentum and energy are replaced by artificial equivalents. In the induction equation, the magnetic-diffusion term is retained, with $\eta$ being replaced by an artificial value. Depending on the simulation, Perfectly Matched Layers (PML: e.g. Berenger, 1994) are also placed at the boundaries that absorb the incoming waves and prevent their spurious reflection and return back to the physical domain. For the best results, 10 to 15 grid points are allocated to the PML layer. This code has been used to study the wave propagation and refraction in a small sunspot (Khomenko and Collados, 2006); non-linear wave propagation, shock formation, mode conversion, and energy transport in small-scale flux tubes with internal structure (Khomenko, Collados, and Felipe, 2008).

Recently, the code was also used to model the propagation of helioseismic waves below the subphotospheric structure of sunspots (Khomenko et al., 2009; Khomenko and Collados, 2009). The most important results obtained for helioseismic wave propagation below sunspots are the following: $i$ ) the fast magnetoacoustic mode represents an analog of quietSun $p$ modes modified by the presence of magnetic field, $i i$ ) helioseismic waves below sunspots are sped up by the magnetic field by $20-40$ seconds compared to the quiet Sun, iii) the magnetic field produces a strong frequency dependence of the travel times, $i v$ ) the eikonal solution gives a qualitatively good approximation for the numerical solution, and finally, $v$ ) the high-frequency fast-mode waves are refracted in the magnetically dominated layers and inject additional energy, possibly causing the power increase observed in acoustic halos surrounding active regions.

\subsubsection{The SLiM Code}

The Semispectral Linear MHD (SLiM) code solves the ideal linearized MHD equations using a spectral expansion in the horizontal directions and a two-step Lax - Wendroff treatment in the vertical. The code includes two absorbing layers at the top and the bottom of the box. In the top layer the waves are heavily damped and the effect of the Lorentz force is systematically reduced. Likewise the bottom layer damps the waves that propagate downward. The code has been tested against analytic solutions, which are described in detail in Cameron, Gizon, and Daiffallah (2007).

In Cameron, Gizon, and Duvall (2008) and Gizon et al. (2009a, 2009b), SLiM was used to study wave propagation through a simplified monolithic model sunspot embedded in a stabilized quiet-Sun model atmosphere (see Section 6.2). The corresponding wave field computed with SLiM was then compared with MDI observations of $f$-and $p$-mode scattering by the sunspot in AR 9787. The comparisons were quite encouraging as the numerical simulations from SLiM were able to reproduce wave absorption and scattering phase shifts. The code has also recently been used on the sunspot model described in Section 4.3.1, as well as 
in simulations where the magnetic field of the sunspot is essentially "switched off", while maintaining the sound speed, pressure, and density perturbations of the sunspot model. This type of simulation is made possible by the fact that the background does not need to be in pressure balance. This type of experiment allows one to disentangle the contributions of the Wilson depression and sound-speed/thermal changes from mode conversion and other magnetic-field effects.

\subsubsection{The SPARC Code}

The Seismic Propagation through Active Regions and Convection (SPARC: Hanasoge, 2010) code uses techniques developed by Hanasoge et al. (2006) and Hanasoge, Duvall, and Couvidat (2007) to simulate helioseismic wave propagation in the near-surface layers of the Sun. Waves are stochastically excited by introducing a forcing term in the vertical momentum equation; the forcing function is prescribed such that a solar-like power spectral distribution is obtained. The solution is temporally evolved using a second-order optimized Runge - Kutta integrator (Hu, 1996). The vertical derivative is resolved using sixthorder, compact, finite differences with fifth-order accurate boundary conditions (Hurlburt and Rucklidge, 2000). The derivatives in the horizontal directions are computed using FFTs (periodic horizontal boundaries). The upper and lower boundaries are lined with damping sponges in order to enhance wave absorption. The SPARC code has been utilized to study wave propagation through model sunspots (e.g. Hanasoge, 2008; Moradi, Hanasoge, and Cally, 2009; Moradi and Hanasoge, 2010), as well as solar convection (Hanasoge, Duvall, and DeRosa, 2010a).

A recent bit of progress with regards to the choice of boundary conditions has been the development of a stable, unsplit PML formulation for the stratified linearized ideal MHD equations. Some related formulations have been developed by Parchevsky and Kosovichev (2007) and Khomenko and Collados (2006). However, instabilities caused by waves at grazing incidence to the boundary prevent long time integrations. By extending the technique of Convolutional Perfectly Matched Layers (C-PML; e.g. Roden and Gedney, 2000), Hanasoge, Komatitsch, and Gizon (2010b) have succeeded in devising a stable C-PML formulation.

\subsubsection{The SAC Code}

In Shelyag et al. (2009), the propagation and dispersion of acoustic waves in a solar-like 2D subphotosphere with localized, non-uniform magnetic-field concentrations was investigated using the Sheffield Advanced Code (SAC) developed by Shelyag, Fedun, and Erdélyi (2008). The numerical code is based on a modified version of the Versatile Advection Code (VAC: Toth, Keppens, and Botchev, 1998), and employs artificial diffusivity and resistivity in order to stabilize the numerical solutions and relies on variable separation to background and perturbed components to treat gravitationally stratified plasma. The complete MHD equations are solved using a fourth-order central difference scheme for the spatial derivatives, and they are advanced in time by implementing a fourth-order Runge-Kutta numerical method.

The standard Model S (Christensen-Dalsgaard et al., 1996) atmosphere is employed as an initial background model, modified so that $\Gamma_{1}$ is kept constant in such a way as to have the adiabatic sound-speed profile closely match the sound speed in Model S. Three different self-similar, non-potential, magnetic-field configurations are employed for the simulations. As the curvature of the magnetic field changes the temperature stratification in the domain, 
the magnetic configurations chosen differ by their field strengths and inclination at the visible solar surface. The acoustic source generates a temporarily localized wave packet with the duration of about 600 seconds, which has a peak frequency of approximately $3.33 \mathrm{mHz}$. The amplitude of the source is of the order of a few centimeters per second. Such a low amplitude makes sure that convective processes will not be initiated in the otherwise convectively unstable equilibrium, and that the perturbations are kept linear, i.e. they do not change the background strongly.

Three cases, a weak strongly-curved magnetic field with $B_{z}=120 \mathrm{G}$ at the surface, a strong weakly curved magnetic field with $B_{z}=3.5 \mathrm{kG}$, and a strongly curved strong magnetic field with $B_{z}=3.5 \mathrm{kG}$ were analyzed by means of time-distance helioseismology. The travel-time dependencies show that for the first bounce the main part of the effect of the magnetic field on the acoustic wave is due to the change of the temperature structure in the sunspot. Nevertheless, the wave-mode conversion from purely acoustic to the slowmagneto-acoustic wave motion, characterized by an energy leak downward, is also observed in the cases with a strong surface magnetic field.

\subsection{Eikonal Methods}

While numerical simulations of wave propagation have significantly aided our level of understanding of helioseismology, further guidance is still needed in setting up the correct numerical experiments and understanding wave propagation in magnetized plasmas. MHD ray theory (Weinberg, 1962) has traditionally provided a very useful conceptual framework in which to understand wave propagation, even though this is questionable at the surface where the pressure and density scales vary rapidly, since the assumption of slowly varying coefficients may not be justified.

Regardless of these shortcomings, however, ray theory has been used in helioseismology for some time, being one of the several methods that have been applied to asymptotic inversions of helioseismic frequency measurements in the past (e.g. Gough, 1984). In general, it has performed well beyond its formal domain of applicability, a prime example being the agreement between the wave-mechanical analysis of Cally (2005), the ray-theory modeling of Cally (2006), and the recent results of Hansen and Cally (2009), who find very good agreement between generalized ray theory and previously published exact solutions (Cally, 2001, 2009b).

Moradi and Cally (2008) recently combined eikonal methods and observational data by constructing a 3D sunspot model based on observed surface magnetic-field profiles to propagate magneto-acoustic rays across the sunspot model for a range of depths to reproduce a skip-distance geometry similar to center-to-annulus cross-correlations used in timedistance helioseismology. In another recently completed work, Moradi, Hanasoge, and Cally (2009) compared the results of forward modeling via MHD ray theory and a 3D ideal MHD solver, concluding that the simulated travel-time shifts were strongly determined by MHD physics and confirmed their strong dependence on the frequency and phase-speed filter parameters used. Khomenko et al. (2009) have similarly applied MHD ray theory to validate and analyse the results of their numerical simulations. In another series of recently published works, Cally (2009a) investigates the direct (magnetic) and indirect (thermal) effects of the magnetic field on vertically propagating waves, suggesting that, overall, travel-time perturbations in umbrae appear to be predominantly thermal, while in penumbrae they are mostly magnetic. Cally (2009b) provides strong evidence for significant phase jumps (or discontinuities) associated with fast magneto-acoustic rays that penetrate the $a=c$ level in sunspots. This effect appears to be more pronounced in highly inclined field characteristic of penumbrae. 
Most of the above work has centered on the study of properties of individual rays propagating through magnetized atmosphere by solving the ray equations for a point source in non-magnetic solar model. In a recent study, Shelyag et al. (2009) have shown the importance of considering the full family of rays corresponding to a particular problem's initial conditions, as these define important geometric properties of the excited wave field such as the wave front, caustics, and phase surfaces.

\section{Update on the Analysis of AR 9787}

\subsection{Travel-Times Comparison: Time-Distance and Helioseismic Holography}

It has been thought by some that travel times computed from time - distance helioseismology (Duvall et al., 1997) should be very similar (or maybe identical) to those measured using helioseismic holography (Braun and Lindsey, 2000). However, a detailed comparison of measured travel times has not been done. We have done such a comparison for an eigh-hour interval for AR 9787. For the time-distance case, correlations are calculated between the central point and the quadrants. For the holography, egression and ingression signals are constructed in a quadrant geometry and correlations are done with the central point. Normal phase-speed filters were used (Couvidat et al., 2005).

Cuts across the travel-time maps for the sunspot are shown in Figure 6 for two distances. Frames (a) and (b) show the distance $24.35 \mathrm{Mm}$ and (c) and (d) show the shortest distance $6.20 \mathrm{Mm}$. The agreement is excellent for the distance of $24.35 \mathrm{Mm}$. However, there are still disagreements for the shortest distances, which we hope to understand soon.

\subsection{Frequency Dependence of One-Way Travel Times}

The dependence of mean and difference travel times on the central frequency of the wave packets measured over a sunspot region was shown and interpreted by Braun and Birch $(2006,2008)$ as an indication of perturbations largely confined to a region not deeper than a few Mm. The largest frequency variations in travel times were seen for the small travel distances $[\Delta]$ which are of the size (diameter) of the spot or smaller. In this section, we conduct a similar study for the sunspot in AR 9787. This type of study is important as, in principle, it should help us to constrain models of the subsurface structure of sunspots.

Apart from a phase-speed filter centered around a phase speed $v_{\mathrm{ph}}=54 \mathrm{~km} \mathrm{~s}^{-1}$ with a width (FWHM) of about $40 \%$ of $v_{\text {ph }}$, we also use Gaussian frequency filters, of $1-\mathrm{mHz}$ width, centered at every $0.25-\mathrm{mHz}$ interval between 2.5 and $5.0 \mathrm{mHz}$ to study the frequency dependencies of the travel times. In Figure 7 we show the umbral averages of perturbations associated with in-going and out-going wave travel times (measured using center-toannulus surface-focus geometry), $\delta \tau_{\text {in }}$ and $\delta \tau_{\text {out }}$, as well as mean travel-time perturbations, $\delta \tau_{\text {mean }}$, against frequency for $\Delta=50 \mathrm{Mm}$. There appears to be a strong frequency dependence associated with both $\delta \tau_{\text {in }}$ and $\delta \tau_{\text {out }}$ measured across the umbra of the sunspot in AR 9787. This result appears to be in good qualitative agreement with the measurements of Braun and Birch (2008), who look at frequency dependence of the travel-time difference, albeit for another sunspot. In particular, see the top right panel of Figure 5 in Braun and Birch (2008), which contains the travel-time difference results for Filter I (distances of 48 $55 \mathrm{Mm})$. 

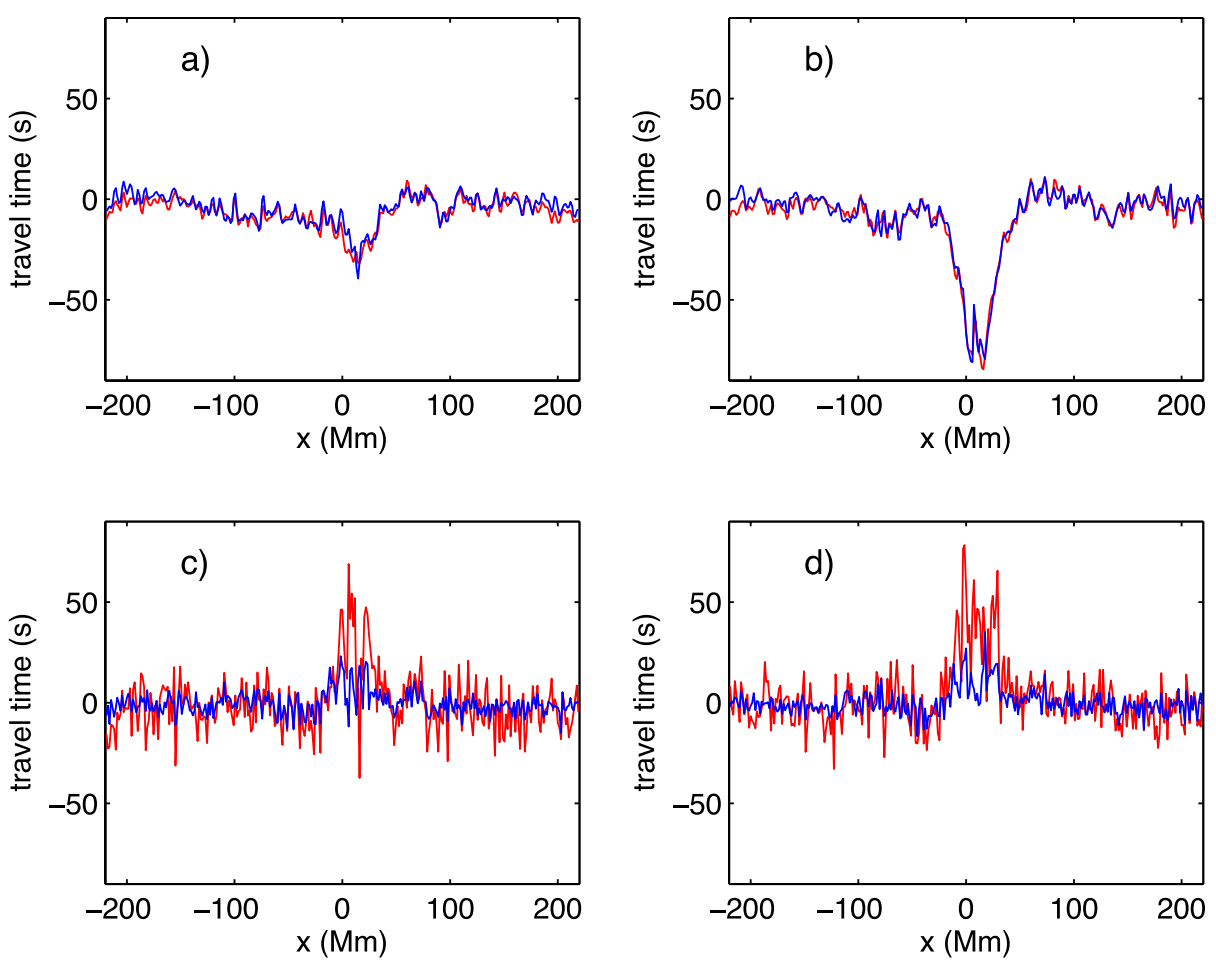

Figure 6 Comparison of travel times computed from helioseismic holography (red) with time-distance helioseismology (blue). Cuts through the center of the sunspot in the East - West direction are shown. (a) Inward-going times, distance of 24.35 Mm. (b) Outward-going times, distance of $24.35 \mathrm{Mm}$. (c) Inward-going times, distance of 6.20 Mm. (d) Outward-going times, distance of $6.20 \mathrm{Mm}$.

Figure 7 Frequency dependence of surface-focus travel times (shown as one-way and mean umbral averages of perturbations) for $\Delta=50 \mathrm{Mm}$, for the sunspot in AR 9787. For clarity, only error bars for mean travel times are shown. The error bars represent standard deviations of travel-time perturbations over the umbral pixels.

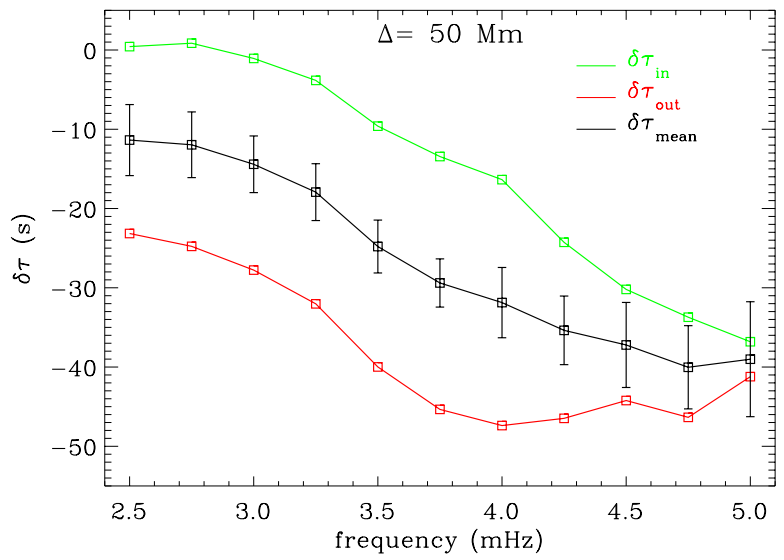

The underlying reason for such a strong frequency dependence of travel times at large distances is not entirely clear, however. The interaction of helioseismic waves with sunspots can be influenced by a number of effects: the vertical extent of the sunspot and the Wilson depression, p-mode absorption (e.g., Braun, Duvall, and Labonte, 1987; Braun, 1995; 
Cally, Crouch, and Braun, 2003), the contribution from subsurface flows, radiative-transfer effects (Rajaguru et al., 2006), and the impact of power reduction and source suppression in sunspots (Rajaguru et al., 2007; Hanasoge et al., 2008; Chou et al., 2009). More detailed analyses and modeling of the sunspot in AR 9787 (e.g. Section 7.8) will be required to isolate these effects and identify the cause(s) of the observed frequency dependence of travel times.

\subsection{Effects of Filtering on Travel Times}

In this section we show a simple toy model for the sensitivity of time-distance travel times to changes in the power spectrum of solar oscillations. We will use this simple model to develop a qualitative understanding of the ridge and inter-ridge measurements (Thompson and Zharkov, 2008) of AR 9787 (Gizon et al., 2009a).

\subsubsection{A Model Power Spectrum}

Before studying the impact of data analysis filters on time-distance measurements, it is necessary to have a model for the power spectrum of solar oscillations. Here we describe a simple model obtained from fitting the azimuthal average (over the angle of the wavevector $\mathbf{k}$ ) of a 24 hour power spectrum from full-disk MDI data from a quiet-Sun region. We then carry out a least-squares fit to the azimuthally averaged power spectrum with a model spectrum of the form

$$
P(k, \omega)=|G(k, \omega)|^{2}+B(k, \omega),
$$

where $k=\|\mathbf{k}\|$ is the horizontal wavenumber, $\omega$ is the temporal frequency, $B$ is the background power, and the function $G$ is given by

$$
G(k, \omega)=\sum_{n=0}^{n_{\max }} a_{n}(k)\left\{\frac{1}{\left[\omega-\omega_{n}(k)\right] \omega_{n}(k)}-\frac{1}{\left[\omega+\omega_{n}^{*}(k)\right] \omega_{n}^{*}(k)}\right\} .
$$

This form of $G$ is motivated by Equations (47) and (48) of Birch, Kosovichev, and Duvall (2004) in the case where the imaginary parts of the mode eigenfunctions are small. The free parameters in the fit are the complex mode frequencies $\left[\omega_{n}(k)\right]$ the mode amplitudes $\left[a_{n}(k)\right]$ and the parameters describing a background power spectrum $[B]$ that is linear in $\omega$ at each value of $k$. Here $n$ is the radial order and $n_{\max }$ is the maximum radial order used in the normal-mode summation. In the examples shown here, $n_{\max }=2$. Throughout this toy model we will work in plane-parallel geometry; this is appropriate as we will be considering distances and wavelengths that are small compared to the solar radius.

\subsubsection{Sensitivity of Travel-Time Shifts to Change in Mode Frequencies}

For problems where the statistics of the wave field are horizontally translation invariant and the expected (limit) power spectrum is azimuthally symmetric, the expectation value of the time-distance cross-covariance $[C(\Delta, t)]$ can be obtained from the limit power spectrum $[P(k, \omega)]$ as

$$
C(\Delta, t)=2 \pi \int_{-\infty}^{\infty} \mathrm{d} \omega \int_{0}^{\infty} k \mathrm{~d} k J_{0}(k \Delta) \mathcal{F}^{2}(k, \omega) P(k, \omega) \mathrm{e}^{-\mathrm{i} \omega t},
$$




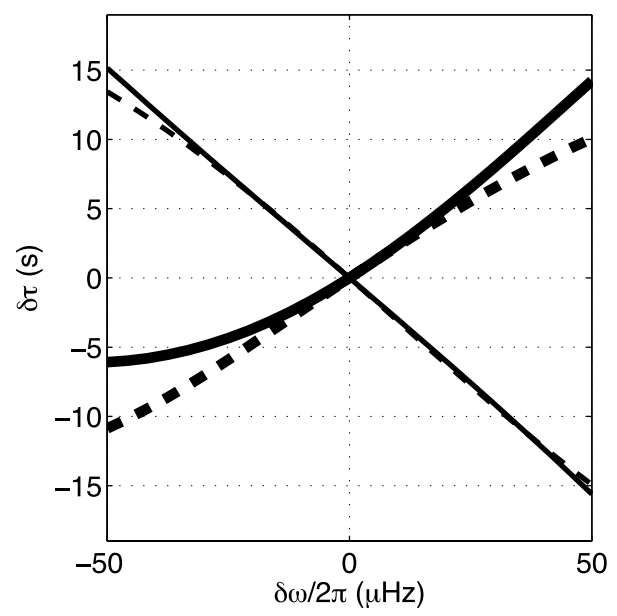

Figure 8 Travel-time shifts for the case of the ridge filter (thin lines) and inter-ridge filter (heavy lines) as functions of the change $[\delta \omega]$ in the real part of the resonance frequency of $n=1$ mode. The solid lines show travel-time shifts obtained from the one-parameter method of Gizon and Birch (2002) and the dashed line show those from the linear definition of Gizon and Birch (2002). In both cases, the fitting window is chosen to be 20 minutes wide and centered at 28 minutes time lag. The travel distance in all cases is $\Delta=16 \mathrm{Mm}$. Notice that for the case of the ridge filter, the two definitions of travel-time shift give very similar results. For the case of the inter-ridge filter, the difference between the two definitions increases with the amplitude of the frequency shift.

where $J_{0}$ is the zeroth-order Bessel function, and $\mathcal{F}$ is the data-analysis filter (e.g. Gizon and Birch, 2002). Using Equation (16), we can compute the cross-covariance that we would expect for any model of the power spectrum.

Travel-time shifts can be obtained from the cross-covariance function using a wide variety of techniques (e.g. Duvall et al., 1997; Gizon and Birch, 2002, 2004). For the examples shown here we use the linear definition of Gizon and Birch (2004) and also the one-parameter fitting method from Gizon and Birch (2002).

Thompson and Zharkov (2008) showed that in sunspots, travel-time shifts measured with ridge filters (filters that isolate power along ridges) and inter-ridge filters (filters that isolate the part of the $k-\omega$ diagram between the ridges) give travel-time shifts (relative to the quiet Sun) of opposite sign. They found that the ridge filters yielded decreased phase-travel times. This would seem to imply that the waves in sunspots have increased phase speed. The same behavior was observed in the case of AR 9787 by Gizon et al. (2009a, 2009b).

As a very highly simplified toy model of this situation, we investigate the travel-time shifts caused by increasing the resonant frequencies in the power spectrum. The reason for choosing increased mode frequencies is to mimic the situation of increased phase speeds (i.e. increased mode frequency at fixed $k$ ). The procedure is as follows: $i$ ) use Equation (16) to compute a reference cross-correlation from the model power spectrum described above, ii) compute a perturbed power spectrum by increasing the model resonance frequencies by a constant value $\delta \omega$, iii) compute a perturbed cross-covariance from this perturbed power spectrum, and $i v$ ) fit the perturbed and reference cross-covariances to obtained the shift in the travel time caused by the change in resonance frequencies.

Figure 8 shows the results of this procedure for two different choices of the filter $\mathcal{F}$ : the $p_{1}$-ridge filter and the inter-ridge filter isolating the region between the $f$ and $p_{1}$ of Thompson and Zharkov (2008). In both cases a 1-mHz bandpass filter centered at $3.5 \mathrm{mHz}$ has also 

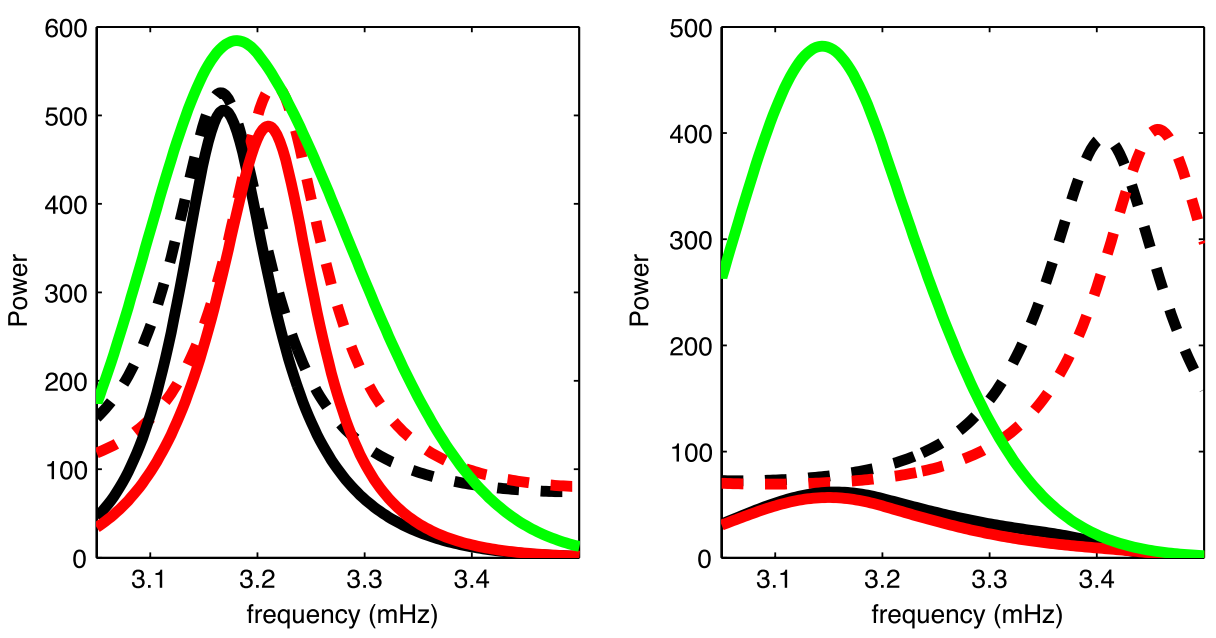

Figure 9 Slices through the filtered and unfiltered power spectra for the ridge filter (left) and inter-ridge filter (right). For the ridge filter case the slice is at $k=1.1 \mathrm{rad} \mathrm{Mm}^{-1}$ and for the inter-ridge case the slice is at $k=0.94 \mathrm{rad} \mathrm{Mm}^{-1}$ (in both cases the slice is near the $k$ of maximum power). In both panels, the red (black) dotted lines show the unfiltered power spectra with (or without) the frequency shift. The green lines show the filter. The solid red (black) lines show the filtered power spectra with (or without) the frequency shift. In the ridge-filter case, the consequence of increasing the mode frequency is that the mean power moves to higher frequency (thus higher phase speed). In the inter-ridge filter case, the mean power moves to lower frequency (lower phase speed).

been applied (Thompson and Zharkov, 2008). In the results shown here we have only considered the impact of changes in the frequency of the $p_{1}$ ridge. For the case of the ridge filter, increases in the mode frequencies (i.e. increased phase speeds for all waves) yield decreased phase times. This is the expected result. For the case of the inter-ridge filter, however, we find that increases in wave speeds yield increased travel times. This is an unintuitive result and in this toy model is due to interaction of the inter-ridge filter with the power between the ridges.

Figure 9 shows slices through power spectra for the two cases. The main effect of the perturbations to the mode frequencies is to move the ridges in the unfiltered power spectra. For the inter-ridge-filtered case, the effect is more subtle as this filter isolates the part of the spectrum that lies between the ridges. The net result of moving the $p_{1}$ ridge to higher frequency and applying the inter-ridge filter is to move power to lower frequency (hence lower phase speed) at fixed $k$.

\subsection{Ring-Diagram Structure Inversions}

The mode frequencies from ring-diagram analysis can be used to determine the thermodynamic structure of the gas under the tracked region. The mode parameters themselves are obtained by fitting a function $\left[P_{n}\left(\omega, k_{x}, k_{y}\right)\right]$ to the $3 \mathrm{D}$ power spectrum.

In this work, we use a fourteen-parameter function defined by Basu, Antia, and Bogart (2004). The fit ridges can be interpolated to wavenumber $k$, and the resulting frequencies $\left[\omega_{n}(k)\right]$ can be treated as normal modes using the techniques of global-mode analysis. Typically, inversions for solar structure are performed by linearizing the stellar oscillation equations around a reference model. Then, the differences in the frequency $\left[\omega_{i}\right]$ of the $i$ th mode 


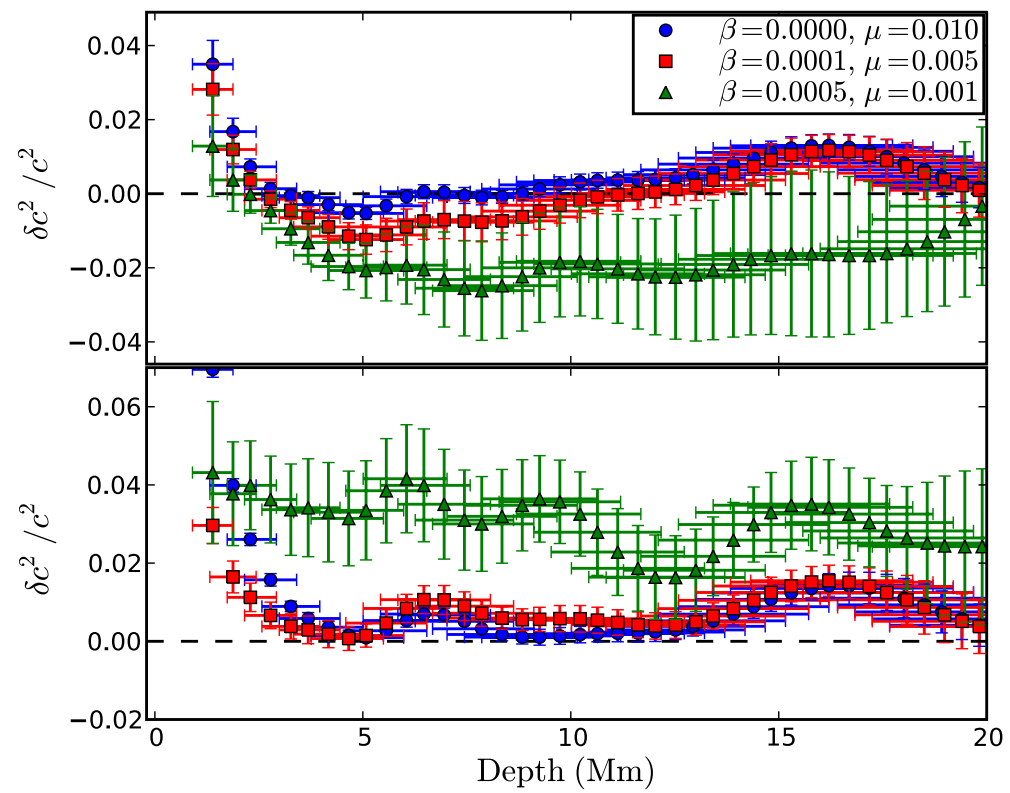

Figure 10 The effects of inversion-parameter choices on inversion results. Three of the four inversion parameters are varied in these inversions - these inversions were performed with a fixed target width of $W=0.0055$. The top panel shows inversions with a surface term removed, and the bottom panel shows inversions without a surface term. In each case, three different inversions are shown with different values of the error-suppression term $[\mu]$ and the cross-term suppression term $[\beta]$.

between the data and reference model, with $i$ representing the pair $(n, k)$, are related to the changes in structure by:

$$
\frac{\delta \omega_{i}}{\omega_{i}}=\int K_{c^{2}}^{i}(z) \frac{\delta c^{2}}{c^{2}}(z) \mathrm{d} z+\int K_{\rho}^{i}(z) \frac{\delta \rho}{\rho}(z) \mathrm{d} z+\frac{F_{\text {surf }}\left(\omega_{i}\right)}{Q_{i}}+\epsilon_{i} .
$$

The surface term $\left[F_{\text {surf }}\left(\omega_{i}\right)\right]$ is a smoothly varying function of frequency that accounts for non-adiabatic effects confined to the surface layers of the Sun and is normalized by the mode inertia $Q_{i}$. The observational errors are given by $\epsilon_{i}$. The inversion kernels $\left[K_{c^{2}}^{i}(z)\right.$ and $K_{\rho}^{i}(z)$ ] are known functions of the reference model, and they give the sensitivity of a mode to changes at a given depth. In a ring-diagram analysis, however, we generally invert relative to mode frequencies measured in a nearby inactive region of the Sun.

The inversion technique used is called Subtractive Optimally Localized Averages (SOLA), a technique pioneered in terrestrial seismology (Backus and Gilbert, 1967). The SOLA method takes a specified target averaging kernel $\left[\mathcal{T}\left(z_{0}, z\right)\right]$ which is localized around a target height $\left[z_{0}\right]$ and minimizes the difference between that target and the actual averaging kernel, along with the contributions from the cross-term kernel and from the errors (e.g. Rabello-Soares, Basu, and Christensen-Dalsgaard, 1999). There are two trade-off parameters: $\mu$, which acts to suppress errors, and $\beta$, which suppresses the cross-term kernel. There are also four free parameters to be chosen in this inversion method: the cross-term suppression term $\beta$, the error suppression term $\mu$, the characteristic width of the target kernel $W$, and $\Lambda$, the number of polynomials used to expand $F_{\text {surf }}$. Structure inversions using ring-diagram modes are quite unstable, and very sensitive to the choice of these parameters. 


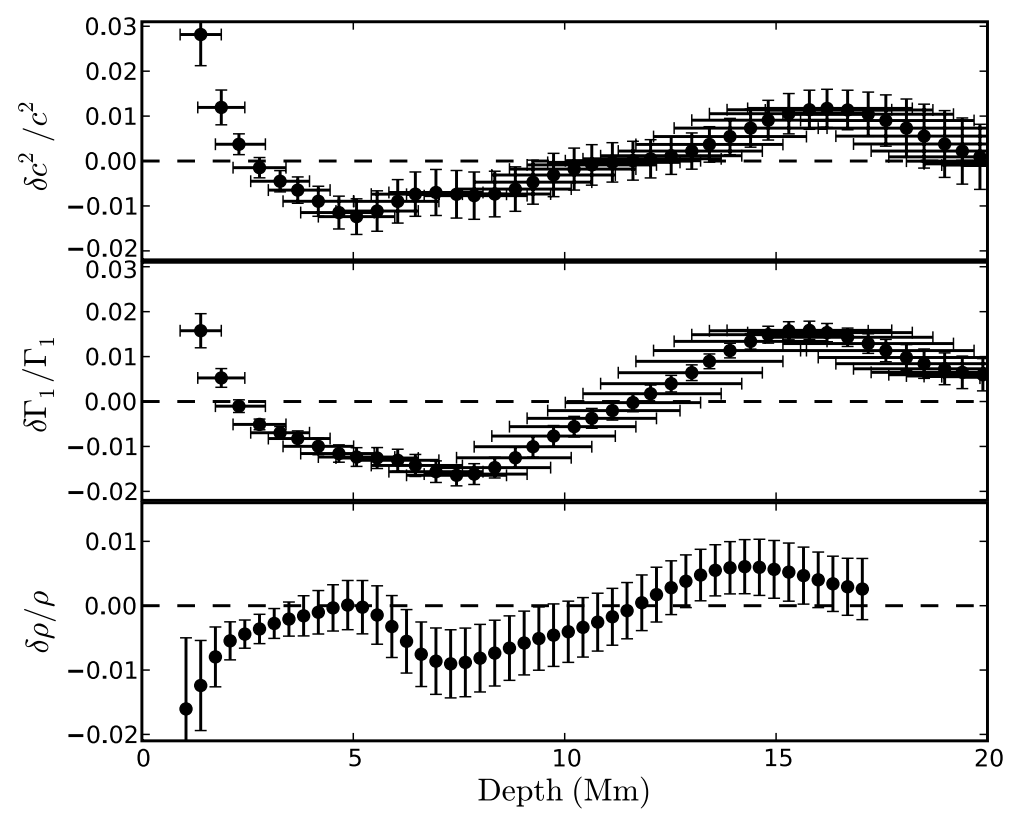

Figure 11 Ring-diagram inversions for the structure beneath AR 9787. Inversions are performed for both sound speed squared $\left[c^{2}\right]$ (top panel) and adiabatic index $\left[\Gamma_{1}\right]$ (middle panel). The inversions are performed relative to a nearby quiet region - the sense of the inversions is active minus quiet. The results for $c^{2}$ and $\Gamma_{1}$ are used to infer a profile for the density $[\rho]$ (bottom panel).

Figure 10 shows sound-speed inversions with a variety of different choices of inversion parameters. The most dramatic choice to be made is whether or not to include a surface term in the inversions, and inversions are shown both with and without a surface term. A good inversion should have a well-localized averaging kernel $\left[\mathcal{K}\left(z_{0}, z\right)\right]$ around a target height $\left[z_{0}\right]$ as well as reasonably small cross-term kernels.

In Gizon et al. (2009a, 2009b), we presented an inversion of the region AR 9787 for the sound speed. In Figure 11, we show an inversion of the same region for sound speed $\left[c^{2}\right]$ and for adiabatic index $\left[\Gamma_{1}\right]$. The inverted quantities are fairly consistent with the results from other active regions (e.g. Basu, Antia, and Bogart, 2004; Bogart et al., 2008). There is a depression in sound speed between approximately $3 \mathrm{Mm}$ and $10 \mathrm{Mm}$ depth. Below that depth, the perturbation becomes positive.

The adiabatic index is depressed below $3 \mathrm{Mm}$, with an enhancement below $12 \mathrm{Mm}$. If $c^{2}$ and $\Gamma_{1}$ are determined, the other thermodynamic quantities are also, in principle, known. In Figure 11, we also show an inferred density profile. The density between approximately $5 \mathrm{Mm}$ and $11 \mathrm{Mm}$ appears to be slightly depressed in the active region compared to the quiet Sun, while the region below that has a lower density than the quiet Sun. There is a depression in density at the shallow edge of the inversion, which might be a sign of a Wilson depression of the optical surface. It should be noted here, however, that results in the shallowest layers of the Sun are quite uncertain, due both to lack of resolution in the inversions and to uncertainties in the physics. 

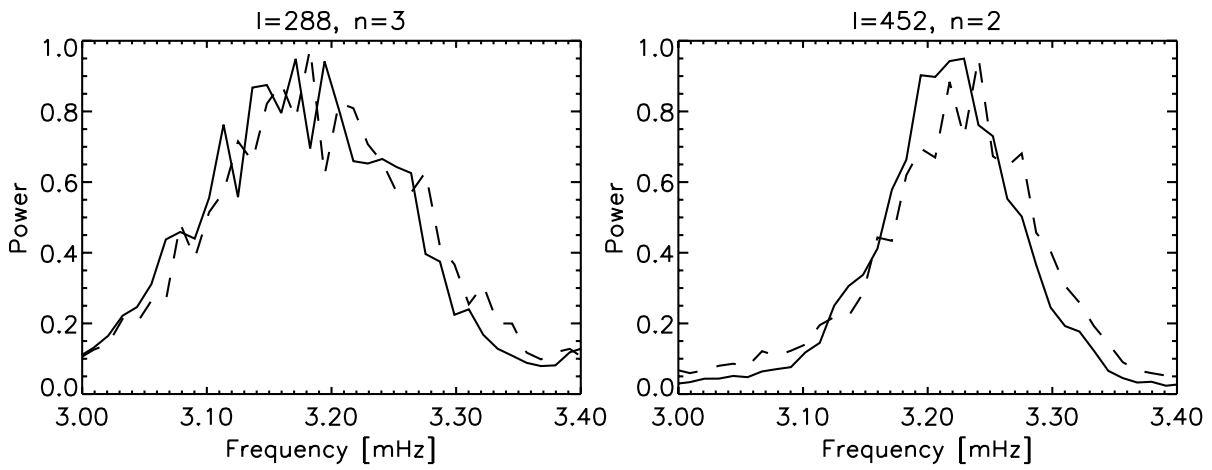

Figure 12 Power spectra for two inward (solid line) and outward (dotted line) propagating $p$ modes. Left: $\ell=288, n=3$; right: $\ell=452, n=2$. The peaks of the outward-propagating modes are shifted to higher frequencies.

\subsection{Moat Flow: Hankel Analysis}

Fourier - Hankel decomposition is a useful analysis tool to study $p$ modes in the vicinity of sunspots. In particular, the method has been used to study the absorption of $p$-mode power by active regions (Braun, Duvall, and Labonte, 1988; Bogdan et al., 1993; Braun, 1995; Chen, Chou, and TON Team, 1996). In this procedure, the wave signal of the $p$ modes is decomposed into inward- and outward-propagating modes in an annular region surrounding a sunspot. The annulus is chosen to be small enough to allow describing the spatial part of the solar oscillations with Hankel functions. In the seismic study of AR 9787 undertaken by Gizon et al. (2009a, 2009b), the power absorption of this particular sunspot was demonstrated by Fourier -Hankel decomposition for the $m=0 p$ modes.

Fourier-Hankel decomposition also turns out to be useful for investigating the horizontal outflow associated with active regions. An outflow directed horizontally (the moat flow) would result in Doppler shifts of the $p$ modes traveling into and out of the spot. A radial flow therefore leaves its signature in the power spectra by shifting the $p$-mode ridges.

Here we extend the Fourier - Hankel analysis of AR 9787 using the procedure described by Braun (1995). For this analysis, the annular region around AR 9787 is defined by an inner radius of $30 \mathrm{Mm}$ and an outer radius of $137 \mathrm{Mm}$ measured from the spot center. Power spectra for inward and outward propagating waves were obtained for modes with azimuthal order $m=-5,-4, \ldots, 5$. Figure 12 displays the resulting spectra for two modes. It is immediately apparent by visual examination of the spectra that the outward-going $p$ mode power is shifted to higher temporal frequencies relative to the inward-going $p$ modes. We note that the power spectra displayed were normalized in order to correct for the power absorption due to the presence of the sunspot. The observed frequency shift is of the order of $10 \mu \mathrm{Hz}$.

\subsection{Ring-Diagram Analysis of Flows}

\subsubsection{Large-Scale Flows Around Active Regions}

Dense-pack ring-diagram analyses of MDI and GONG++ data have shown that subsurface flows associated with active regions have the following characteristics:

- Active regions are surrounded by extended inflows and outflows, depending on depth. 
- Subsurface flows of active regions are twisted.

- Active regions rotate faster than quiet regions.

- Flux emergence correlates with the flow-divergence signal.

The horizontal components of solar subsurface flows are determined over a range of depths from the surface to about $16 \mathrm{Mm}$ using the dense-pack ring-diagram analysis (Haber et al., 2002). Daily flow maps are calculated for 189 dense-pack regions of $15^{\circ}$ diameter with centers spaced by $7.5^{\circ}$ in latitude and central-meridian distance. The dense-pack technique thus measures flows on horizontal scales comparable to the size of active regions.

Locations of strong active regions show, on average, extended, divergent horizontal flows at depths greater than about $10-12 \mathrm{Mm}$ and convergent horizontal flows closer to the surface (both with an amplitude of about $50 \mathrm{~m} \mathrm{~s}^{-1}$ ). AR 9787 shows this pattern as well (Figure 13). Using a mass-conservation constraint, ring analysis infers vertical flows associated with the horizontal flows. The convergent and divergent flow pattern is one of the most consistent characteristics of subsurface flows associated with active regions. It has been studied with several helioseismic techniques (Gizon, Duvall, and Larsen, 2001; Braun, Birch, and Lindsey, 2004; Haber et al., 2002, 2004; Zhao and Kosovichev, 2004; Komm et al., 2005). These extended flows, however, should not be confused with the much more localized moat flow (divergent, $250 \mathrm{~m} \mathrm{~s}^{-1}$, see next section and Gizon et al., 2009a, 2009b), which cannot be resolved with $15^{\circ}$-aperture ring analysis.

Active regions are locations of flows with larger vorticity values; they are locations of strong vertical gradients of the horizontal flows. Strong active regions are identifiable by a dipolar pattern in zonal and meridional vorticity (Mason et al., 2006) and AR 9787 appears to be no exception (Figure 13). The presence of active regions is barely noticeable in vertical-vorticity maps of this spatial resolution. However, active regions are, on average, characterized by cyclonic vorticity (counter-clockwise in the northern hemisphere), which might be due to the Coriolis force acting on the flows (Spruit, 2003). This agrees with observations with higher spatial resolution (Duvall and Gizon, 2000; Gizon and Duvall, 2003; Zhao and Kosovichev, 2004).

From direct surface measurements, it is well known that active regions rotate faster than quiet ones. This has been confirmed with helioseismology (Gizon, 2004; Zhao and Kosovichev, 2004). A ring-diagram analysis of about six years of GONG++ data shows that the average zonal flow of active regions is about $4 \mathrm{~m} \mathrm{~s}^{-1}$ larger than that of quiet regions from the surface to a depth of $16 \mathrm{Mm}$ (Komm et al., 2009b). The difference is about one order of magnitude smaller than that derived from surface measurements of active and quiet regions, which is most likely a consequence of the rather large size of the dense-pack patches and the resulting averaging over many different types of magnetic features. Results from acoustic holography and time-distance analysis with higher horizontal resolution support this interpretation (Braun, Birch, and Lindsey, 2004; Gizon, 2004; Zhao, Kosovichev, and Duvall, 2004).

A survey of 788 active regions observed with GONG++ makes it possible to determine a signature of emerging magnetic flux in subsurface flows associated with active regions (Komm, Howe, and Hill, 2009a). At depths greater than about $10 \mathrm{Mm}$, upflows become stronger with time when new flux emerges. At layers shallower than about $4 \mathrm{Mm}$, the flows might start to change from downflows to upflows, when flux emerges, and then back to downflows after the active regions are established. The flow response to emerging flux agrees with numerical simulations of emerging flux tubes (Fan, 2001; Schüssler and Rempel, 2005) where upflows indicate the beginning of flux emergence and surface cooling due to adiabatic expansion leads to downflows along the emerged loops. 


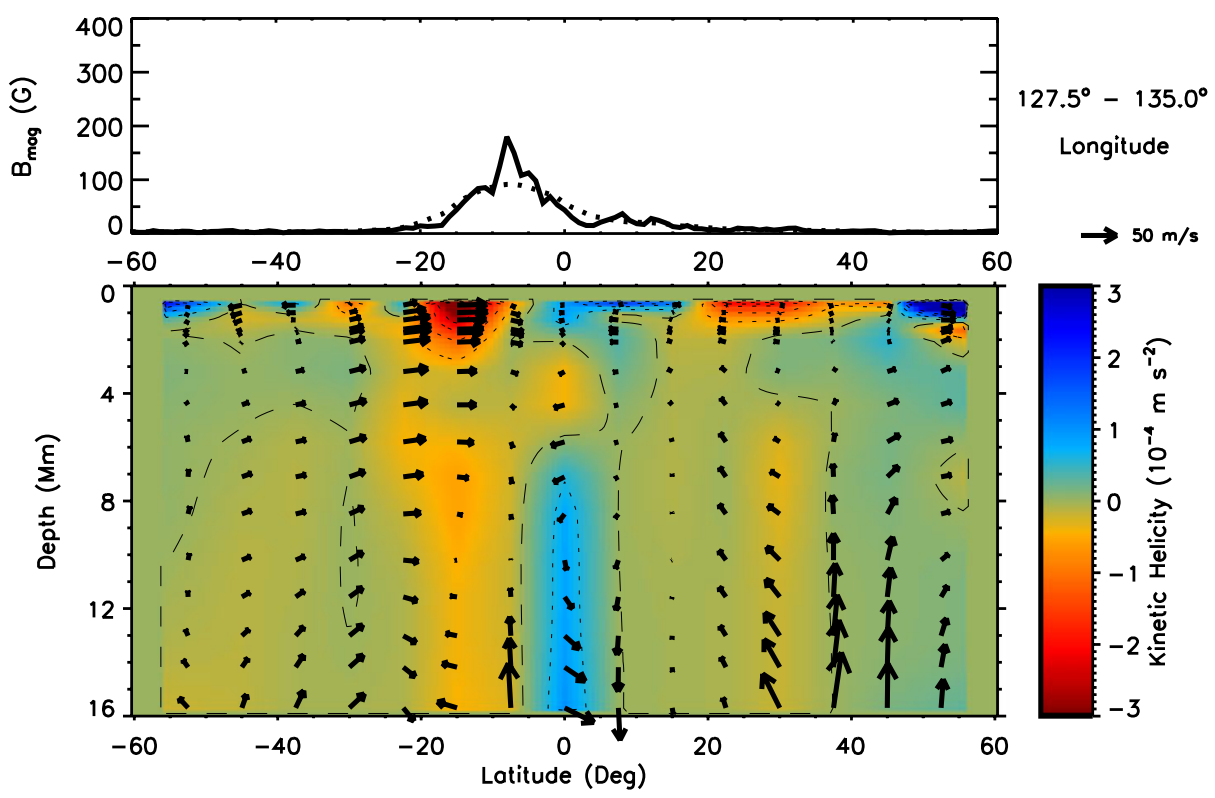

Figure 13 Top: The unsigned magnetic flux (solid line) at $127.5^{\circ}-135.0^{\circ}$ longitude as a function of latitude and binned over $15^{\circ}$ (dotted line). Bottom: The kinetic-helicity density at $127.5^{\circ}-135.0^{\circ}$ longitude as a function of latitude and depth. The kinetic-helicity density is the scalar product of the velocity and vorticity vector. The arrows represent the meridional and vertical-velocity components with the vertical one increased by a factor of ten for visibility. Average zonal and meridional flows have been subtracted. Active region 9787 is noticeable as the location of strong helicity values of opposite sign, which coincides with the peak of the unsigned magnetic flux. The region shows upflows at depths greater than about $10 \mathrm{Mm}$ and downflows at shallower depths at grid points of $-15^{\circ}$ to $-7.5^{\circ}$ latitude.

\subsubsection{Sunspot Flows from Small Rings}

Subsurface horizontal flows determined by high-resolution ring analysis (HRRA) for the four days when AR 9787 was closest to the center of the disk, show characteristic outflows from the lone sunspot corresponding to moat flows (Figure 14).

The analysis was carried out on $2^{\circ}$-diameter tiles whose centers were spaced $1^{\circ}$ apart. Each flow arrow represents a spatial average over an entire tile and is itself the result of averaging the flows determined from all of the fitted $f$ modes of the power spectrum for that tile since there are not enough modes to perform a true inversion. This means that the flows are characteristic of the gas from the surface down to a depth of $2 \mathrm{Mm}$, the region where the $f$ modes reside. The four-day panel (Figure 14) shows the evolution of largescale zones of divergence around the sunspot corresponding to large supergranules as well as a seeming twist of the flows coming from the sunspot on 25 January. The flows are overplotted on averaged MDI magnetograms for the given day where the green and red colors show magnetic fields of opposite polarity (the green area corresponding to the sunspot).

Similar subsurface flow signatures were obtained by Gizon et al. (2009a, 2009b), who used $f$ to $p_{4}$ ridge-filtered time - distance travel times to produce linear inversions for flows (see Jackiewicz, Gizon, and Birch, 2008) around the sunspot in AR 9787. Figure 15 shows a summary plot of the subsurface horizontal flows around the sunspot, which appear very 

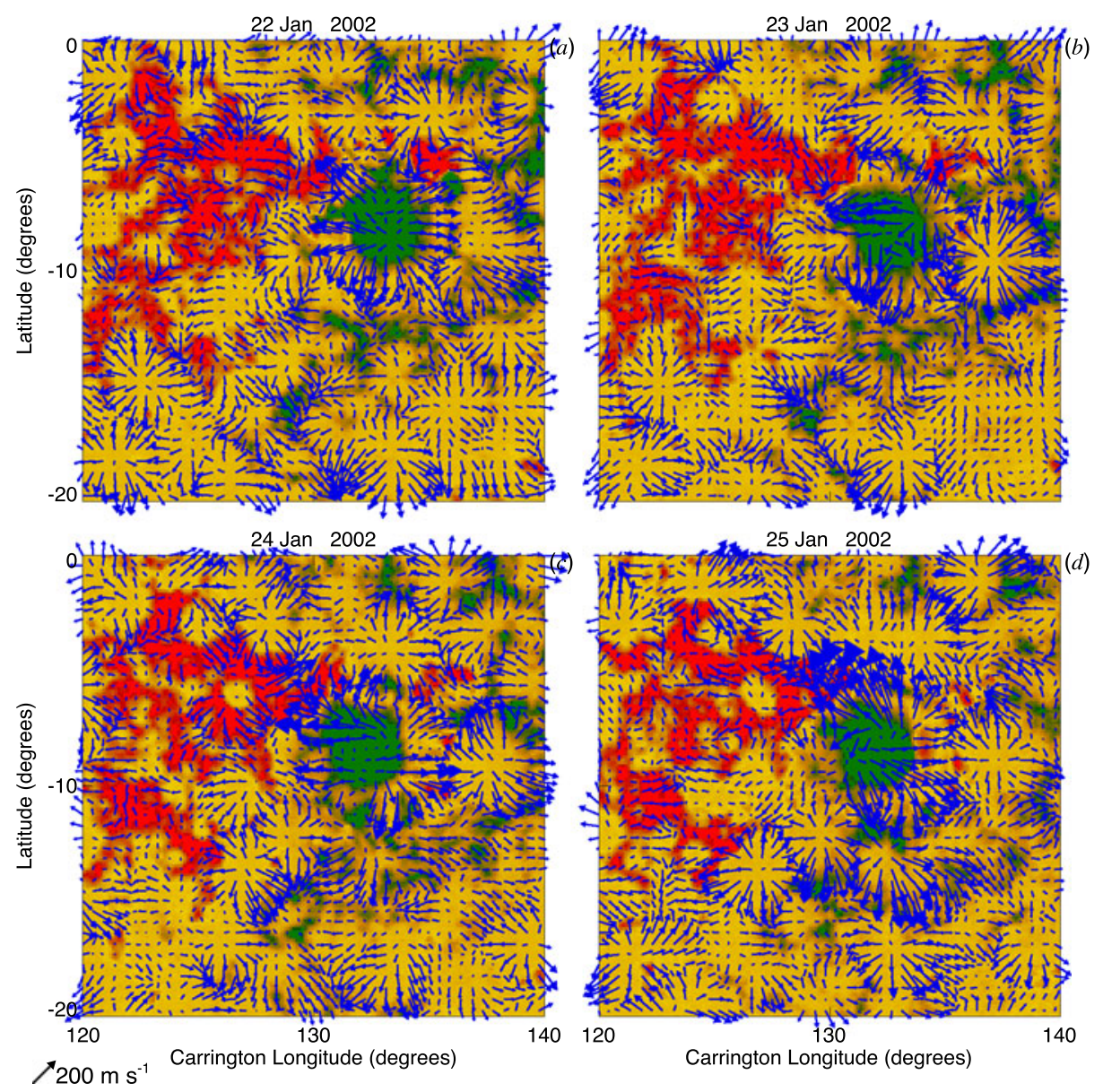

Figure 14 Subsurface flows using high-resolution ring analysis of full-disk MDI Dynamics Doppler data from 22-25 January 2002. MDI magnetograms are underlaid with red and green specifying opposite polarities of the magnetic field. The flows were determined from $f$-mode data and are thus representative of flows within the top $2 \mathrm{Mm}$ of the convection zone. The outflows from the sunspot (shown in green) correspond to moat flows, while the cells of divergence seen in the vicinity of the active region correspond to larger supergranular scales of about 50-60 Mm. The magnetic network often appears at the edges of these larger cells.

consistent with the flows detected by HRAA, and direct observations of the moat flow (see Section 2.2.5).

\subsection{Acoustic Halos}

\subsubsection{Observations}

The acoustic halo is an observed enhancement of high-frequency (i.e. above the acoustic cut-off frequency at approximately $5.3 \mathrm{mHz}$ ) acoustic power surrounding regions of strong magnetic field. Hindman and Brown (1998) demonstrate that this enhanced power at high frequencies tends to be prominent in intermediate magnetic-field strengths of $50-250 \mathrm{G}$ 
Figure 15 Map of horizontal flows (arrows) at a depth of $1 \mathrm{Mm}$ around the sunspot in AR 9787 using one day of MDI full-disk data and time-distance helioseismology on 24 January (Gizon et al., 2009a). The spatial resolution is determined by the width of the averaging kernel (FWHM $7 \mathrm{Mm}$ ) shown in the top-left corner. The longest arrow corresponds to a flow of $450 \mathrm{~m} \mathrm{~s}^{-1}$. The surface line-of-sight magnetic field is displayed in red and blue shades (saturated at $\pm 350 \mathrm{G}$ ). This inversion uses the $f$ and $p_{1}$ through $p_{4}$ modes; it is discussed by Jackiewicz, Gizon, and Birch (2008).

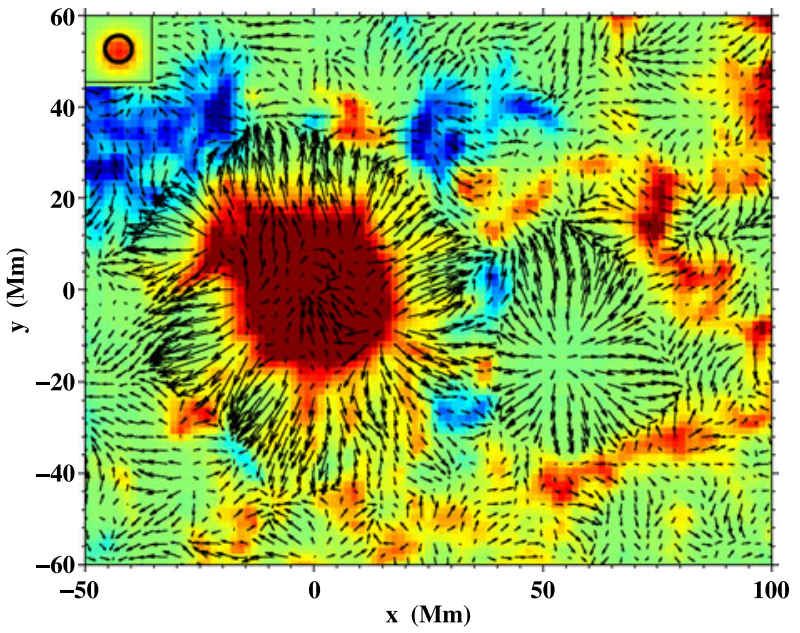

and appears to be absent in the equivalent continuum intensity observations. In Gizon et al. (2009a, 2009b) it was demonstrated that AR 9787 has an extensive plage region, with the excess power at above $5.5 \mathrm{mHz}$ being spatially correlated with these plage regions. It was also noticed that the strongest regions of acoustic halo occur between regions of opposite polarity.

For our analysis, we calculate the vector magnetic field at the surface from the MDI magnetograms using a potential magnetic-field extrapolation. From this we define the inclination of the magnetic field from vertical $[\gamma]$. Figure 16 shows the average normalized power against total magnetic-field strength for $\gamma<45^{\circ}$ (solid black) and $\gamma>45^{\circ}$ (dashed blue) for $1-\mathrm{mHz}$ frequency band passes centered at $3,4,5$, and $6 \mathrm{mHz}$ as indicated. This highlights the importance of the field inclination for the acoustic halo.

In another recent analysis, Schunker and Braun (2010) further demonstrate that the acoustic halo is specifically confined to intermediate field strengths (between $100 \mathrm{G}$ and $400 \mathrm{G}$ ) with $60^{\circ}<\gamma<120^{\circ}$ from vertical. The power confined to this range of inclination has a peak which increases in frequency from $5.5 \mathrm{mHz}$ to $6.5 \mathrm{mHz}$ as the magnetic-field strength increases from $100 \mathrm{G}$ to $400 \mathrm{G}$. In addition, they also find that the radial-order ridges of the azimuthally summed power spectrum are shifted to higher wavenumbers than the quiet Sun at constant frequency in the halo regions. The reason for this is, as yet, unexplained.

\subsubsection{Theories}

A growing number of theories attempt to explain the phenomenon of wave-velocity enhancements in the vicinity of active regions. The hypothesis of an altered wave excitation mechanism due to the presence of magnetic fields was proposed by Brown et al. (1992) and Braun et al. (1992) and revisited more recently by Jacoutot et al. (2008). Of course, it is also equally likely that the substantial differences in the wave-propagation physics in active regions could also be leading to these enhancements, a possibility noted by a number of authors (e.g. Hindman and Brown, 1998; Donea, Braun, and Lindsey, 1999; Moretti et al., 2007).

From analyses of simulations of waves propagation through a model sunspot, Hanasoge (2008) demonstrated that it was possible to obtain these halos without the requirement of 


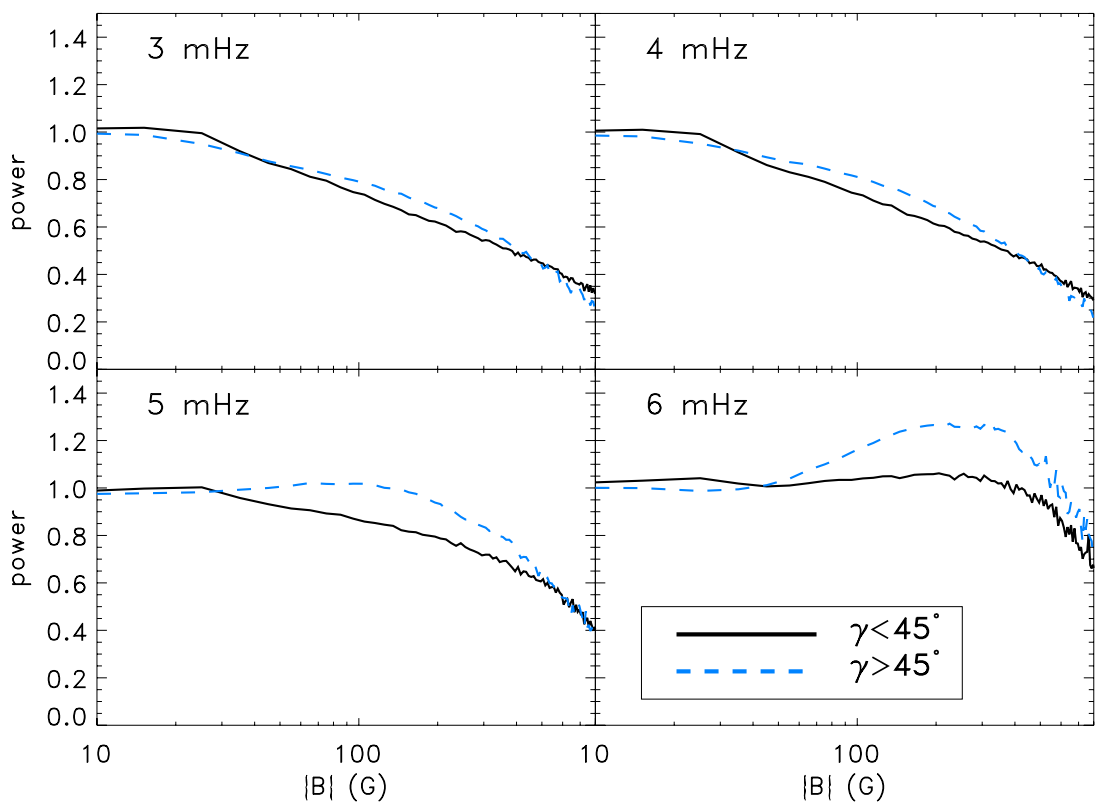

Figure 16 Average normalized power against total magnetic-field strength (in bins of $10 \mathrm{G}$ ) for inclination $\gamma<45^{\circ}$ (solid black) and $\gamma>45^{\circ}$ (dashed blue) in frequency band passes as indicated. The importance of more horizontal field where $\gamma>45^{\circ}$ for the acoustic halo is evident.

enhanced sources. A wave-scattering theory based on pure hydrodynamics was also put forth by Kuridze et al. (2008, 2009); their thesis was that bipolar canopies create a secondary trapping region for the outward-propagating high-frequency waves. Alternately, Khomenko and Collados (2009) have suggested that upward propagating high-frequency waves undergo conversion to fast modes, refract off the large Alfvén-speed gradient in the atmosphere, and return to the photosphere. This results in the same wave being observed twice, once while going upward, and the second time on its way back down, thereby causing an enhancement. Finally, Hanasoge (2009) has proposed that the presence of a large number of wave scatterers in the vicinity of active regions may cause preferential scattering into low mode-mass waves, i.e. waves whose energy is focused in the near-surface layers. Relative to the original (quietSun) set of modes, this scattered configuration contains stronger surface-velocity signatures (i.e. lower net mode mass), thereby leading to the halos. Observationally, it can be confirmed that the velocity enhancements are primarily present at high $\ell$, in the region of the power spectrum where the modes possess the lowest mode masses.

In reality, it may well be that there are multiple mechanisms at play, i.e. altered wave excitation, reflection/refraction of waves by magnetic fields, and preferential scattering into waves with low mode masses. Further studies are required before any solid conclusions can be drawn.

\subsection{Comparison of Observed Cross-Covariances with Simulations}

Cameron, Gizon, and Duvall (2008) and Gizon et al. (2009a, 2009b) showed that the crosscovariance of the MDI Doppler velocity is a very useful quantity to study the scattering of solar waves by the sunspot in AR 9787. The cross-covariance between two points is closely related to the Green's function between these two points (e.g. Gizon, Birch, and Spruit, 2010, 
and references therein). By extension, the cross-covariance between the signal averaged over a line and any other point can be used to study the interaction of plane-wave packets with the sunspot.

The left panels in Figure 17 show such cross-covariances at particular values of the correlation time lag, after the wave packets have traversed the sunspot. While Gizon et al. (2009a, 2009b) had studied only $f$-mode wave packets, here we also show the observed cross-covariances for $p_{1}$ and $p_{2}$ wave packets. The wave packets are constructed using standard ridge filters (wave power peaks near $3 \mathrm{mHz}$ ). The observed cross-covariances show that, in all cases, solar waves speed up through the sunspot and that their amplitudes are reduced relative to the quiet Sun. The stochastic noise in the observations is reduced by exploiting all available symmetries, including the near-cylindrical symmetry of the sunspot.

The cross-covariances also provide very strong observational constraints to test the validity of sunspot models. Here we have used the SLiM code to test the semi-empirical model of AR 9787 by Cameron et al. (2010) (see Section 4.3.1 and Figure 4) by propagating $f, p_{1}$, and $p_{2}$ wave packets through the sunspot model. The initial conditions of the simulations are chosen such that the vertical component of velocity in the simulation is directly comparable with the cross-covariance function. As can be seen in Figure 17, the simulations reproduce the basic features of the observations. This indicates that the sunspot model has a seismic signature that is close to the real sunspot, which is very encouraging.

\section{Discussion and Perspective}

\subsection{Sunspot Structure: A Critical Assessment of Existing Models}

Recent years have seen an increased interest in questions relating to the structure of sunspots, fueled by a fortunate coincidence of several factors. After decades of more gradual improvements, classical (visible light) observations have quickly improved in quality over the past few years. With the Swedish 1-m Solar Telescope, spatial resolution achieved has made a jump (with a resolution of $0.1^{\prime \prime}$ achieved regularly in the blue), while temporal coverage has vastly improved through the long time sequences from the Hinode satellite. Secondly, helioseismic observations have now approached the point where they can be turned into powerful diagnostics of spot structure, as discussed elsewhere in this article. Finally, and perhaps most dramatically, the realism achieved by $3 \mathrm{D}$ radiative MHD simulations has now opened the perspective of a definitive physical interpretation of the phenomenology of sunspot structure, including umbral dots, light bridges, penumbral filaments, the Evershed flow, the moat flow, and their relations to each other. It is likely that understanding will soon replace the historically evolved patchwork of mutually inconsistent views and physically dubious $a d$ hoc models. The following gives a brief perspective on these developments. For more detail, see the reviews in Scharmer (2009) and Nordlund and Scharmer (2010).

As discussed in Section 3, the structure of a sunspot as observed at the surface is kept together by forces deeper down. Assuming this anchoring at depth as given (but yet to be explained), one can seek a physical interpretation of the surface structure observed in umbrae and penumbrae. At the observed field strengths, $1-3 \mathrm{kG}$, the magnetic field is strong enough to suppress convection. This explains why spots are dark but it does not explain the particular time-dependent fine structure observed.

In early observational work (e.g. Mamadazimov, 1972) the mix of long dark and bright filaments has been interpreted as showing a magnetic field (dark, as in the umbra) on top of the normal photosphere (shining through in the bright filaments). This explained the general 

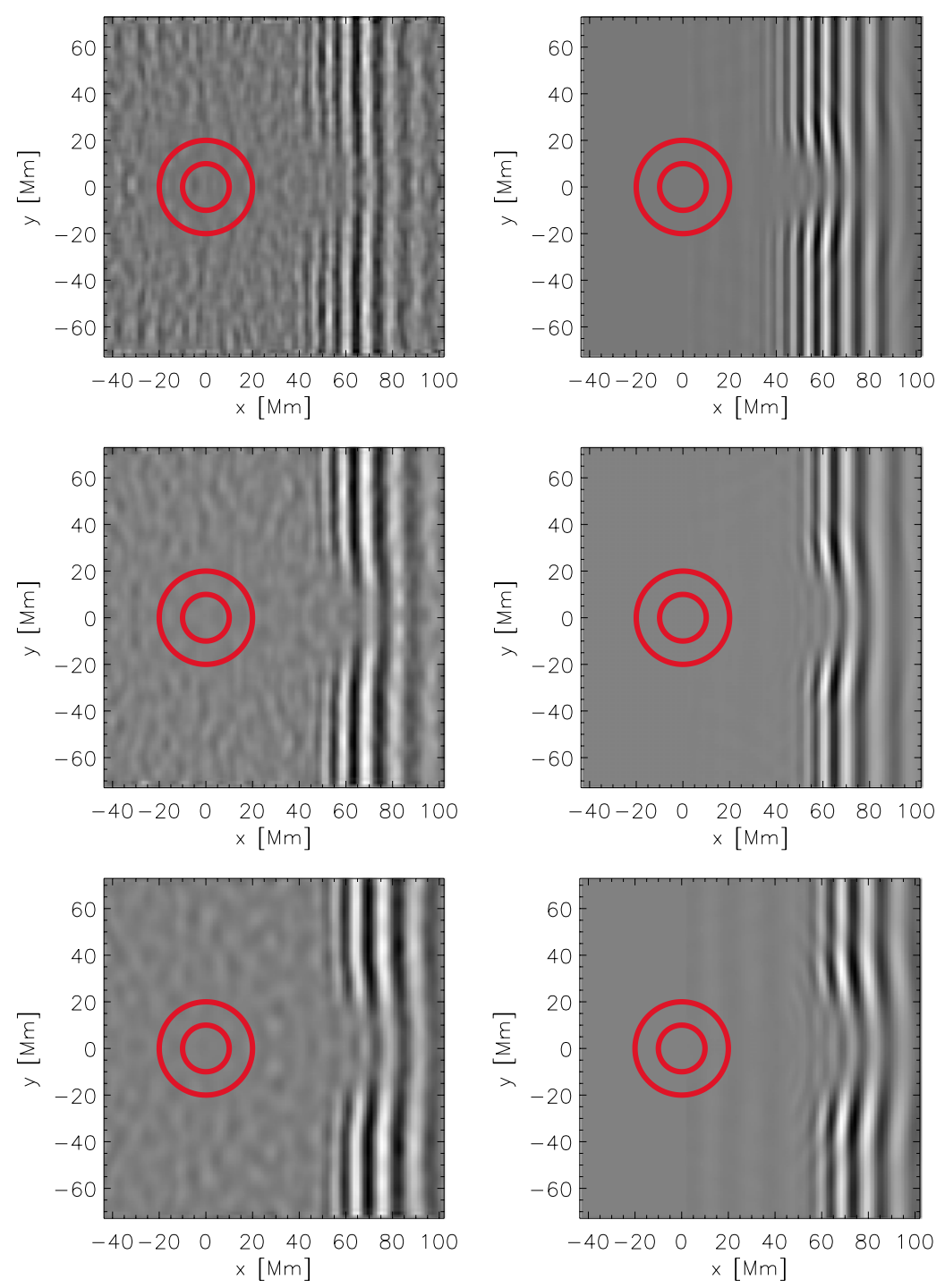

Figure 17 Left panels: Observed MDI cross-covariance functions between the Doppler velocity averaged over the line $x=-40 \mathrm{Mm}$ and the Doppler velocity at any other point $(x, y)$ in the map. The sunspot AR 9787 is located at the origin and the circles show the boundaries of the umbra and penumbra. The cross-covariance is averaged over seven days. The top-left panel shows the cross-covariance for the $f$-mode ridge at time lag $t=185$ minutes, the middle-left panel for the $p_{1}$-mode ridge at time lag $t=165$ minutes, and bottom panel for the $p_{2}$-mode ridge at time lag $t=145$ minutes. The right panels show SLiM simulations of the propagation of $f, p_{1}$, and $p_{2}$ wave packets in the $+x$-direction through the semi-empirical sunspot model of Cameron et al. (2010). The vertical velocity in the simulation is directly comparable to the observed cross-covariances.

appearance of the penumbra and the nearly photospheric brightness of the bright structures, but required that the penumbral magnetic field only touches over the photosphere (a "thin penumbra", with optical depth only of order unity). The field would have to be essentially horizontal in the penumbra, field lines crossing the solar surface only in the umbra. This does 
not agree with the observed inclination of the penumbral field. In fact, most of the magnetic flux of a spot crosses the surface through the penumbra, not the umbra. The penumbral field, on average, is therefore not horizontal, and the observed field must continue to some depth below the surface.

This has led to interpretations in terms of convection in a magnetic field extending to a substantial depth below the surface (a "thick penumbra"). An influential conceptual picture was the Danielson (1961) model of convective "rolls": an overturning flow in a plane perpendicular to a horizontal magnetic field. This idea has led to a "magnetoconvection" view of the penumbra, which interprets the observed filamentary structures as turbulent fluctuations in a mean magnetic field inclined at some angle to the solar surface (e.g. Tildesley and Weiss, 2004 and references therein). It also plays a role as a conceptual picture underlying global models of sunspot structure, such as the one due to Jahn and Schmidt (1994).

A key aspect of any realistic model of magnetic surface structure is the transition from the gas-pressure dominated regime below the photosphere to the magnetically dominated atmosphere. In the penumbra this takes place just at the photospheric level. The transition takes place over a couple of pressure scale heights. This is no more than the horizontal resolution of most telescopes, and comparable to the smallest horizontal structures seen in the penumbra. With photospheric observations one is thus looking at the thin interface between two physically different regimes, rather than a slice through some quasi-uniform turbulent medium. The consequences of this fact have not been realized in most of the currently popular views.

Observations of this interface show a mix of magnetic-field strengths and inclinations. This has led to the idea of inclusions ("flux tubes") embedded in a background field of different direction (Solanki and Montavon, 1993). This has become the dominant theme in interpretations of penumbral structure (e.g. Martínez Pillet, 2000; Bellot Rubio et al., 2003b; Bellot Rubio, Balthasar, and Collados, 2004; Borrero, 2007).

In the atmosphere, however, where $B^{2} / 8 \pi \gg P$, forces other than magnetic are small. The magnetic field in such a case is nearly force free. This provides strong restrictions on the physically realizable field configurations. These constraints are actually violated in most proposed ideas about penumbral structure, especially the floating flux tubes in the embedding and uncombed type models. An exception is the proposal by Martens et al. (1996), who propose a structure of magnetic sheets of different directions separated by forcefree currents.

Force-free, but not current-free, configurations also cause problems, however. The strength of the magnetic field decreases away from the spot. A force-free field has the property that a difference in field-line direction between neighboring magnetic surfaces increases with decreasing field strength along the field (this is related to the magnetic torque being conserved along field lines; see the discussion in Spruit and Scharmer, 2006). Force-free models thus predict that differences in inclination should become more prominent with height above the photosphere and distance from the umbra. Observations in the chromosphere (the "superpenumbra") do not support this. Instead, magnetic-field-line directions traced by chromospheric indicators appear to become more uniform with height. This is not consistent with force-free models like those of Martens et al. (1996). Instead, these observations indicate that the field in the atmosphere is much closer to a potential (current-free) configuration.

The "gappy penumbra" model (Spruit and Scharmer, 2006; Scharmer and Spruit, 2006), based on the cluster model of a sunspot, explains how the pattern of strong irregularities decreasing with height above the surface comes about naturally in a potential field, as a re- 
sult of gaps between field lines created below the surface by overturning convection. ${ }^{1}$ At the same time this model addresses the long-standing heat-flow problem: the question of how the observed radiative energy flux is supplied to the penumbral surface. The low field strength in the gaps allows convection to supply the heat flux emitted by the surface unimpeded by magnetic forces. Convection in magnetic rolls, on the other hand, does not solve this problem, since a roll with the diameter of a penumbral filament does not contain enough thermal energy to maintain the radiative flux over the life of a filament. However, the gappy model has problems explaining the structure in the outer penumbra, where observations show a nearly horizontal field (also confirmed in the numerical simulations of Rempel et al., 2009b), and the presence of returning magnetic flux (Section 2.2.1).

Apart from the gappy model, a number of other physical models for the penumbral currently exist. The model of Schlichenmaier, Jahn, and Schmidt (1998) (see also Schlichenmaier, 2009 and references therein), which has the virtue of substantial physical detail, prescribes that magnetic-flux tubes rising from below the surface carry heat as well as an outward flow, interpreted as the Evershed flow. It has problems reproducing the observed heat flux from the penumbra, however, and the postulated rising flux tubes have not been identified in the numerical simulations. In the numerical simulations, both the heat flux and the Evershed flow are found to be driven by overturning convection, not in the form of magnetic rolls or flux tubes, but in regions low field strength (Nordlund and Scharmer, 2010).

Another proposal appeals to the process of "turbulent flux-pumping" (Thomas et al., 2002; Weiss et al., 2004). In this process asymmetry between upflows and downflows in stratified convection effectively transports horizontal magnetic-field lines downward. Penumbral structure is then ascribed to this process. The source of the penumbral structure is said to be located outside the penumbra (Weiss et al., 2004). Alternatively, it has also been implied to be located in the penumbra itself (Tildesley and Weiss, 2004). The turbulentpumping mechanism appears to offer a plausible mechanism for producing the returning magnetic flux in the outer penumbra, as well as the apparent hysteresis observed in the transition between a pore and a sunspot (Weiss et al., 2004). However, there are also objections to this model that are based on the fact that the action of convection on a magnetic field overlying it, as in the outer penumbra, would be expected to have the opposite effect of turbulent pumping. Overturning convection would instead expel a magnetic field that is imposed from above, through the well-known process of convective expulsion (Zel'dovich, 1956; Weiss, 1966). The process is seen in action in numerical simulations of horizontal magnetic fields overlying granulation by Steiner et al. (2008, 2009).

As the quality of observations continues to improve, realistic, 3D, radiative, numerical MHD simulations of sunspots (Schüssler and Vögler, 2006; Heinemann et al., 2007; Scharmer, Nordlund, and Heinemann, 2008; Rempel, Schüssler, and Knölker, 2009a; Rempel et al., 2009b) show a remarkable level of agreement with these observations. The agreement includes the properties of umbral dots, the inward-propagating bright filaments, the dark cores overlying them, the varying aspect of penumbral structure with viewing angle, the varying field strengths and direction in penumbral filaments, the dependence of these on height, the moat flow, and the Evershed flow. We expect that more advanced simulations

\footnotetext{
${ }^{1}$ By "overturning" we mean here the convective pattern also observed in realistic simulations of stellar surface convection (see the review by Scharmer, 2009 for more detail). As opposed to traditional views approximating convection as closed cells or "rolls", almost nothing of the descending part of the flow returns to the surface, but it is replaced by upflows from larger depths. As opposed to the field-aligned rolls of the Danielson (1961) kind, this pattern maintains a low field strength in the gaps through the process of convective expulsion. While these gaps may not be exactly field free (as none of the convection zone is), they are regions where the hydrodynamic forces dominate over magnetic forces, i.e., the field is "below equipartition with convection".
} 
in the near future will further improve our understanding of penumbrae, in particular with regard to their outermost parts. With this level of agreement with observations, there is little doubt that the simulations are reproducing the physics of sunspot structure as observed at the surface.

Next to the treatment of the atmospheric magnetic field, the physics of radiation is of equal importance for realism in numerical simulations. Cooling by radiation at the surface determines the thermal structure of the penumbra and drives the observed flows. On the other hand, it also determines the detailed appearance of penumbral structure at the optical depth unity surface. Any physically meaningful comparison with observations thus requires inclusion of radiation physics at a fairly well-developed level. The fact that the level of realism needed for a meaningful interpretation of solar-surface structure, magnetic or quiet, has now been achieved should be considered one of the most significant advance in theoretical solar physics of the past few decades.

\subsection{The Starspot Connection}

It is important to keep in mind that our Sun is not the only star that possesses spots. Moreover, it is more than likely that all late-type stars with convective envelopes exhibit spots, or "starspots" (e.g. reviews by Berdyugina, 2005 and Strassmeier, 2009). Spotted stars constitute roughly $90 \%$ of all stars in the Milky Way, basically all GKM stars and a large fraction of $\mathrm{F}$, and $\mathrm{L}$ and $\mathrm{T}$ dwarfs are spotted, representing a mass range from the brown dwarf limit up to the $2.4 M_{\odot}$ of a $\mathrm{G}$ giant, and an age range from the pre-main sequence phase up to the asymptotic giant branch. Stars with planets can also be affected by magnetic processes and their magnetic environment may even affect close-in planets and back-react onto the star (see e.g. Shkolnik, Walker, and Bohlender, 2003; Catala et al., 2007; Kashyap, Drake, and Saar, 2008; Lanza, 2008). Therefore, we must understand sunspots first, before we can understand starspots.

Models of starspots do not exist yet but the mere size difference suggests that a scaling from a sunspot model is not appropriate (e.g. Solanki and Unruh, 2004). While sunspots typically cover $10^{-4}$ to $10^{-5}$ of the solar surface and only during solar maximum reach about $10^{-3}$, the record holder among other stars is still the one big starspot seen on the K0 giant XX Tri in December 1997 (Strassmeier, 1999). Based on the star's Hipparcos distance of $197 \mathrm{pc}$, the spot's area covers approximately $12 \times 20 R_{\odot}^{2}$, i.e. $22 \%$ of the star's hemisphere or 10000 times the area of the largest sunspot group. Clearly, its emergence, structure, and decay must be addressed on a global scale. Figure 18 shows a Zeeman-Doppler image of the active spotted star II Pegasi (Carroll et al., 2007) combined with a numerical simulation of a non-axisymmetric dynamo. Although it is just a demonstration example, it nonetheless highlights the expected global starspot-dynamo connection.

\subsection{Conflicting Helioseismic Observations}

A prevalent, largely phenomenological, approach to modeling the subsurface structure of sunspots has been to treat the regions of magnetism as perturbations to the background wave speed. These types of models have been constructed using a variety of localhelioseismic procedures. A comparison of structural (wave-speed) inversions for AR 9787 using both ring-diagram analysis and time - distance helioseismology with phase-speed filters was presented by Gizon et al. (2009a, 2009b), and it is partially reproduced in Figure 19. 
Figure 18 A starspot on the K subgiant II Pegasi. The surface temperature and the photospheric magnetic field is a Zeeman - Doppler observation while the interior shows a numerical simulation of a non-axisymmetric dynamo in a fully convective star. There, polarity in the western hemisphere mirrors the polarity in the eastern hemisphere. On the surface, red means a field strength of up to $800 \mathrm{G}$ according to the color bar while blue means basically no detection. In the interior, red means the field is pointing outward, blue means the field points inward (Carroll et al., 2007).
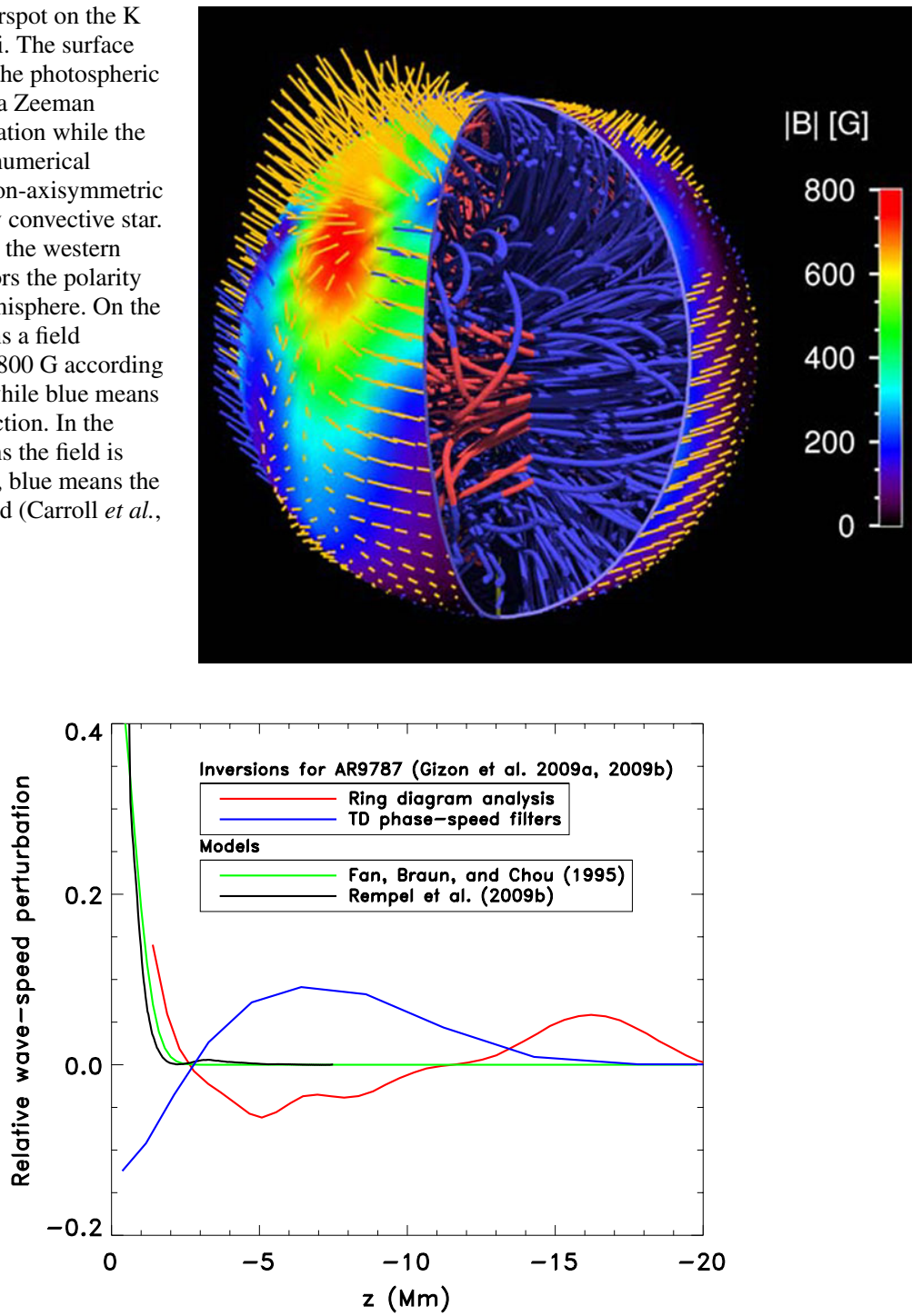

Figure 19 Comparison of different helioseismic methods used to infer wave-speed perturbations below AR $9787\left[\delta c_{w} / c\right]$. The red and blue curves show the ring-diagram and phase-speed filtered time-distance results, respectively, from Gizon et al. (2009a, 2009b). The time-distance result is shown along the axis of the sunspot; the ring-diagram results have been scaled by a factor of ten. The black curve indicates the fast-mode speed perturbation $\left[\left(c_{\mathrm{f}}-c\right) / c\right]$ from the radiative MHD simulations of Rempel et al. (2009b), described in Section 4.4; it approaches the value 75 at $z=0 \mathrm{Mm}$. The green line represents the on-axis wave-speed perturbations deduced from the phenomenological model of Fan, Braun, and Chou (1995), based on the Hankel phase-shift measurements of Braun (1995).

As is evident, these two inversions give subsurface wave-speed profiles with opposite signs and different amplitudes. Gizon et al. (2009a, 2009b) discussed a number of factors which could contribute to such a disagreement, including the observation that the ring-diagram inversions include a treatment of near-surface effects absent from the time- 
distance analyses and the apparent sensitivity of the measurements to details of the analysis which are not included in the models. A prominent example is the sensitivity of timedistance measurements to parameters of the filters (Section 7.3). As suggested by Braun and Birch (2008), some evidence of a strong near-surface contribution to helioseismic measurements in sunspots is shown in the forward modeling of Fourier-Hankel measurements performed by Fan, Braun, and Chou (1995). It is worthwhile, for comparative purposes therefore, to plot the results of the subsurface perturbation suggested by Fan, Braun, and Chou (1995) in Figure 19. An important caveat in this comparison is that the Hankel phaseshift measurements (Braun, 1995), from which this wave-speed result was inferred, were of different sunspots. However, Braun and Birch (2008) show that these phase-shift measurements agree favorably with more recent ridge-filtered holography measurements of sunspots of similar size. The relative fast-wave-speed perturbations of the sunspot model of Rempel et al. (2009b), presented in Section 4.4, are also included in Figure 19 for reference: this model also displays positive wave-speed perturbations in the first $2 \mathrm{Mm}$ below the surface.

\subsection{Emergence of a New Paradigm in Sunspot Seismology}

Keeping in mind all of the above caveats, it is worth noting that three out of four curves shown in Figure 19 are consistent with a strong, positive wave-speed perturbation extending about $2.5 \mathrm{Mm}$ below the surface. Below this depth, the helioseismic inversions show considerably stronger deep wave-speed perturbations (albeit, of opposite signs) than the other methods. Braun and Birch (2006) have argued that shallow wave-speed perturbations such as those suggested by Fan, Braun, and Chou (1995) are required to explain the systematic frequency dependence in the mean travel times observed in sunspots. Of course, it is well to keep in mind the warnings of Gizon et al. (2009a, 2009b), namely the possible naivety of modeling potentially complicated effects of the magnetic field in terms of an equivalent sound-speed perturbation.

Many of these magnetic effects are more suitably explored through the numerical methods discussed in Section 6. Perhaps the strongest argument in favor of a shallow, fast wavespeed model is provided by the linear simulations of MHD wave propagation (Section 7.8). These forward numerical computations show that a simple semi-empirical sunspot model extending no deeper than $2 \mathrm{Mm}$ (Figure 3) is capable of reproducing many features of the helioseismic measurements, in particular the cross-covariance signatures of the sunspot in AR 9787. While additional linear simulations will be needed to confirm this claim, the realistic radiative simulations of sunspot-like structure by Rempel et al. (2009b) will provide the ultimate testbed to validate the forward and inverse methods of sunspot seismology.

Finally, we note that sunspot seismology will benefit greatly from improved observations by the Solar Dynamics Observatory (SDO). The Helioseismic and Magnetic Imager (HMI) instrument onboard SDO will not only provide higher-resolution Doppler images of the solar disk, but it will also provide full vector magnetograms. Reliable measurements of the photospheric magnetic field will be key to constraining the near-surface layers of sunspot models, as detailed models of the surface layers are a necessity in order to probe the deeper structure of sunspots.

Acknowledgements This parametric study of sunspot models is supported by the European Research Council under the European Community's Seventh Framework Programme (FP7/2007-2013)/ERC grant agreement \#210949, "Seismic Imaging of the Solar Interior", to PI L. Gizon (Milestone \#3). The followup analysis of AR 9787 was carried out at the Third HELAS Local Helioseismology Workshop, which was held in Berlin on 12-15 May 2009 and supported by the European Commission under the Sixth Framework Program of the European Union. Portions of this work were also supported by the NASA SDO Science 
Center and Heliophysics Guest Investigator programs through contracts NNH09CE41C and NNG07EI51C to NWRA under PI D.C. Braun. The authors would also like to acknowledge Kaori Nagashima and Takashi Sekii for providing the Hinode observation used in Figure 1, and the anonymous referee for useful comments that helped improve the paper.

\section{References}

Albregtsen, F., Maltby, P.: 1978, Nature 274, 41. doi:10.1038/274041a0.

Albregtsen, F., Maltby, P.: 1981, Solar Phys. 71, 269. doi:10.1007/BF00167551.

Avrett, E.H.: 1981, In: Cram, L.E., Thomas, J.H. (eds.) The Physics of Sunspots, Sacramento Peak Observatory, Sunspot, 235.

Ayres, T.R.: 1996, In: Strassmeier, K.G., Linsky, J.L. (eds.) Stellar Surface Structure, IAU Sympos. 176, Kluwer, Dordrecht, 371.

Babcock, H.W.: 1963, Ann. Rev. Astron. Astrophys. 1, 41. doi:10.1146/annurev.aa.01.090163.000353.

Backus, G.E., Gilbert, J.F.: 1967, Geophys. J. Int. 13, 247. doi:10.1111/j.1365-246X.1967.tb02159.x.

Balthasar, H., Schmidt, W.: 1993, Astron. Astrophys. 279, 243.

Balthasar, H., Wöhl, H.: 1983, Solar Phys. 88, 71. doi:10.1007/BF00196178.

Balthasar, H., Schleicher, H., Bendlin, C., Volkmer, R.: 1996, Astron. Astrophys. 315, 603.

Basu, S., Antia, H.M., Bogart, R.S.: 2004, Astrophys. J. 610, 1157. doi:10.1086/421843.

Baumann, I., Schmitt, D., Schüssler, M., Solanki, S.K.: 2004, Astron. Astrophys. 426, 1075. doi:10.1051/0004-6361:20048024.

Beck, C.: 2008, Astron. Astrophys. 480, 825. doi:10.1051/0004-6361:20078409.

Beckers, J.M., Schröter, E.H.: 1969, Solar Phys. 10, 384. doi:10.1007/BF00145526.

Bellot Rubio, L.R.: 2003, In: Trujillo-Bueno, J., Sanchez Almeida, J. (eds.) Solar Polarization CS-307, Astron. Soc. Pacific, San Francisco, 301.

Bellot Rubio, L.R., Balthasar, H., Collados, M.: 2004, Astron. Astrophys. 427, 319. doi:10.1051/0004-6361: 20041277.

Bellot Rubio, L.R., Balthasar, H., Collados, M., Schlichenmaier, R.: 2003a, Astron. Astrophys. 403 , L47. doi:10.1051/0004-6361:20030576.

Bellot Rubio, L.R., Balthasar, H., Collados, M., Schlichenmaier, R.: 2003b, Astron. Astrophys. 403, L47. doi:10.1051/0004-6361:20030576.

Berdyugina, S.V.: 2005, Living Rev. Solar Phys. 2, 8, http://solarphysics.livingreviews.org/Articles/lrsp-2005-8/.

Berenger, J.P.: 1994, J. Comput. Phys. 114, 185. doi:10.1006/jcph.1994.1159.

Birch, A.C., Kosovichev, A.G., Duvall, T.L. Jr.: 2004, Astrophys. J. 608, 580. doi:10.1086/386361.

Birch, A.C., Braun, D.C., Hanasoge, S.M., Cameron, R.: 2009, Solar Phys. $254,17$. doi:10.1007/s11207-008-9282-9.

Bogart, R.S., Basu, S., Rabello-Soares, M.C., Antia, H.M.: 2008, Solar Phys. $251,439$. doi:10.1007/s11207-008-9213-9.

Bogdan, T.J.: 1999, Astrophys. J. 512, 471. doi:10.1086/306749.

Bogdan, T.J., Brown, T.M., Lites, B.W., Thomas, J.H.: 1993, Astrophys. J. 406, 723. doi:10.1086/172483.

Borrero, J.M.: 2007, Astron. Astrophys. 471, 967. doi:10.1051/0004-6361:20066776.

Borrero, J.M., Solanki, S.K., Bellot Rubio, L.R., Lagg, A., Mathew, S.K.: 2004, Astron. Astrophys. $422,1093$. doi:10.1051/0004-6361:20041001.

Borrero, J.M., Lagg, A., Solanki, S.K., Collados, M.: 2005, Astron. Astrophys. 436, 333. doi:10.1051/0004-6361:20042553.

Borrero, J.M., Solanki, S.K., Lagg, A., Socas-Navarro, H., Lites, B.: 2006, Astron. Astrophys. 450, 383. doi:10.1051/0004-6361:20054378.

Brants, J.J., Zwaan, C.: 1982, Solar Phys. 80, 251. doi:10.1007/BF00147972.

Braun, D.C.: 1995, Astrophys. J. 451, 859. doi:10.1086/176272.

Braun, D.C., Birch, A.C.: 2006, Astrophys. J. Lett. 647, L187. doi:10.1086/507450.

Braun, D.C., Birch, A.C.: 2008, Solar Phys. 251, 267. doi:10.1007/s11207-008-9152-5.

Braun, D.C., Lindsey, C.: 2000, Solar Phys. 192, 307.

Braun, D.C., Duvall, T.L. Jr., Labonte, B.J.: 1987, Astrophys. J. Lett. 319, L27. doi:10.1086/184949.

Braun, D.C., Duvall, T.L. Jr., Labonte, B.J.: 1988, Astrophys. J. 335, 1015. doi:10.1086/166988.

Braun, D.C., Birch, A.C., Lindsey, C.: 2004, In: Danesy, D. (ed.) SOHO 14 Helio- and Asteroseismology: Towards a Golden Future SP-559, ESA, Noordwijk, 337.

Braun, D.C., Duvall, T.L. Jr., Labonte, B.J., Jefferies, S.M., Harvey, J.W., Pomerantz, M.A.: 1992, Astrophys. J. Lett. 391, L113. doi:10.1086/186410. 
Bray, R.J., Loughhead, R.E.: 1964, Sunspots, Chapman Hall, London.

Brickhouse, N.S., Labonte, B.J.: 1988, Solar Phys. 115, 43. doi:10.1007/BF00146229.

Brown, T.M., Bogdan, T.J., Lites, B.W., Thomas, J.H.: 1992, Astrophys. J. Lett. $394, \quad$ L65. doi:10.1086/186473.

Caccin, B., Gomez, M.T., Severino, G.: 1993, Astron. Astrophys. 276, 219.

Caligari, P., Moreno-Insertis, F., Schüssler, M.: 1995, Astrophys. J. 441, 886. doi:10.1086/175410.

Caligari, P., Schüssler, M., Moreno-Insertis, F.: 1998, Astrophys. J. 502, 481. doi:10.1086/305875.

Cally, P.S.: 1991, J. Comput. Phys. 93, 411. doi:10.1016/0021-9991(91)90192-N.

Cally, P.S.: 2001, Astrophys. J. 548, 473. doi:10.1086/318675.

Cally, P.S.: 2005, Mon. Not. Roy. Astron. Soc. 358, 353. doi:10.1111/j.1365-2966.2005.08742.x.

Cally, P.S.: 2006, Phil. Trans. Roy. Soc. Lond. Ser. A 364, 333.

Cally, P.S.: 2009a, Mon. Not. Roy. Astron. Soc. 395, 1309. doi:10.1111/j.1365-2966.2009.14708.x.

Cally, P.S.: 2009b, Solar Phys. 254, 241. doi:10.1007/s11207-008-9290-9.

Cally, P.S., Crouch, A.D., Braun, D.C.: 2003, Mon. Not. Roy. Astron. Soc. 346, 381. doi:10.1046/j.13652966.2003.07019.x.

Cameron, R., Gizon, L., Daiffallah, K.: 2007, Astron. Nachr. 328, 313. doi:10.1002/asna.200610736.

Cameron, R., Gizon, L., Duvall, T.L. Jr.: 2008, Solar Phys. 251, 291.

Cameron, R., Schunker, H., Gizon, L., Pietarila, A.: 2010, Solar Phys. doi:10.1007/a11207-010-9631-3.

Carroll, T.A., Kopf, M., Ilyin, I., Strassmeier, K.G.: 2007, Astron. Nachr. 328, 1043. doi:10.1002/asna. 200710884.

Catala, C., Donati, J.F., Shkolnik, E., Bohlender, D., Alecian, E.: 2007, Mon. Not. Roy. Astron. Soc. 374, L42. doi:10.1111/j.1745-3933.2006.00261.x.

Caunt, S.E., Korpi, M.J.: 2001, Astron. Astrophys. 369, 706. doi:10.1051/0004-6361:20010157.

Chen, K.-R., Chou, D.-Y., TON Team: 1996, Astrophys. J. 465, 985. doi:10.1086/177484.

Cheung, M.C.M., Schüssler, M., Moreno-Insertis, F.: 2007, Astron. Astrophys. 467, 703. doi:10.1051/ 0004-6361:20077048.

Chitre, S.M.: 1963, Mon. Not. Roy. Astron. Soc. 126, 431.

Chitre, S.M., Shaviv, G.: 1967, Solar Phys. 2, 150. doi:10.1007/BF00155915.

Chou, D.Y., Sun, M.T., Chang, H.K.: 2000, Astrophys. J. 532, 622. doi:10.1086/308571.

Chou, D., Liang, Z., Yang, M., Zhao, H., Sun, M.: 2009, Astrophys. J. Lett. 696, L106. doi:10.1088/0004-637X/696/1/L106.

Christensen-Dalsgaard, J., Dappen, W., Ajukov, S.V., Anderson, E.R., Antia, H.M., Basu, S., Baturin, V.A., Berthomieu, G., Chaboyer, B., Chitre, S.M., Cox, A.N., Demarque, P., Donatowicz, J., Dziembowski, W.A., Gabriel, M., Gough, D.O., Guenther, D.B., Guzik, J.A., Harvey, J.W., Hill, F., Houdek, G., Iglesias, C.A., Kosovichev, A.G., Leibacher, J.W., Morel, P., Proffitt, C.R., Provost, J., Reiter, J., Rhodes, E.J. Jr., Rogers, F.J., Roxburgh, I.W., Thompson, M.J., Ulrich, R.K.: 1996, Science 272, 1286.

Collados, M., Martínez Pillet, V., Ruiz Cobo, B., del Toro Iniesta, J.C., Vazquez, M.: 1994, Astron. Astrophys. 291, 622 .

Couvidat, S., Birch, A.C., Kosovichev, A.G., Zhao, J.: 2004, Astrophys. J. 607, 554. doi:10.1086/383342.

Couvidat, S., Gizon, L., Birch, A.C., Larsen, R.M., Kosovichev, A.G.: 2005, Astrophys. J. Suppl. 158, 217. doi:10.1086/430423.

Cowling, T.G.: 1946, Mon. Not. Roy. Astron. Soc. 106, 218.

Cowling, T.G.: 1953, In: Kuiper, G.P. (ed.) The Sun, University of Chicago, Chicago, 532.

Cowling, T.G.: 1957, Magnetohydrodynamics, Interscience, New York.

Cowling, T.G.: 1976, Magnetohydrodynamics, Adam Hilger, Bristol.

Crouch, A.D., Cally, P.S., Charbonneau, P., Braun, D.C., Desjardins, M.: 2005, Mon. Not. Roy. Astron. Soc. 363, 1188. doi:10.1111/j.1365-2966.2005.09515.x.

Danielson, R.E.: 1961, Astrophys. J. 134, 289. doi:10.1086/147159.

Deinzer, W.: 1965, Astrophys. J. 141, 548. doi:10.1086/148144.

del Toro Iniesta, J.C., Tarbell, T.D., Ruiz Cobo, B.: 1994, Astrophys. J. 436, 400. doi:10.1086/174914.

Ding, M.D., Fang, C.: 1989, Astron. Astrophys. 225, 204.

Donea, A.C., Braun, D.C., Lindsey, C.: 1999, Astrophys. J. Lett. 513, L143. doi:10.1086/311915.

D’Silva, S., Choudhuri, A.R.: 1993, Astron. Astrophys. 272, 621.

Duvall, T.L. Jr., Gizon, L.: 2000, Solar Phys. 192, 177.

Duvall, T.L. Jr., Kosovichev, A.G., Scherrer, P.H., Bogart, R.S., Bush, R.I., De Forest, C., Hoeksema, J.T., Schou, J., Saba, J.L.R., Tarbell, T.D., Title, A.M., Wolfson, C.J., Milford, P.N.: 1997, Solar Phys. 170, 63.

Duvall, T.L. Jr., D’Silva, S., Jefferies, S.M., Harvey, J.W., Schou, J.: 1996, Nature 379, 235.

Fan, Y.: 2001, Astrophys. J. Lett. 554, L111. doi:10.1086/320935.

Fan, Y., Braun, D.C., Chou, D.Y.: 1995, Astrophys. J. 451, 877.

Fan, Y., Fisher, G.H., McClymont, A.N.: 1994, Astrophys. J. 436, 907. doi:10.1086/174967. 
Fla, T., Skumanich, A., Osherovich, V.A.: 1982, Astrophys. J. 261, 700. doi:10.1086/160380.

Gizon, L.: 2004, Solar Phys. 224, 217. doi:10.1007/s11207-005-4983-9.

Gizon, L., Birch, A.C.: 2002, Astrophys. J. 571, 966.

Gizon, L., Birch, A.C.: 2004, Astrophys. J. 614, 472. doi:10.1086/423367.

Gizon, L., Birch, A.C.: 2005, Living Rev. Solar Phys. 2, 6. http://solarphysics.livingreviews.org/Articles/ Irsp-2005-6/.

Gizon, L., Duvall, T.L. Jr.: 2003, In: Sawaya-Lacoste, H. (ed.) GONG+ 2002. Local and Global Helioseismology: The Present and Future SP-517, ESA, Noordwijk, 43.

Gizon, L., Duvall, T.L. Jr., Larsen, R.M.: 2000, J. Astrophys. Astron. 21, 339.

Gizon, L., Duvall, T.L. Jr., Larsen, R.M.: 2001, In: Brekke, P., Fleck, B., Gurman, J.B. (eds.) Recent Insights into the Physics of the Sun and Heliosphere: Highlights from SOHO and Other Space Missions, IAU Sympos. 203, Astron. Soc. Pacific, San Francisco, 189.

Gizon, L., Hanasoge, S.M., Birch, A.C.: 2006, Astrophys. J. 643, 549. doi:10.1086/502623.

Gizon, L., Birch, A.C., Spruit, H.C.: 2010, Ann. Rev. Astron. Astrophys. 48, 289. doi:10.1146/annurev-astro082708-101722.

Gizon, L., Schunker, H., Baldner, C.S., Basu, S., Birch, A.C., Bogart, R.S., Braun, D.C., Cameron, R., Duvall, T.L., Hanasoge, S.M., Jackiewicz, J., Roth, M., Stahn, T., Thompson, M.J., Zharkov, S.: 2009a, Space Sci. Rev. 144, 249. doi:10.1007/s11214-008-9466-5.

Gizon, L., Schunker, H., Baldner, C.S., Basu, S., Birch, A.C., Bogart, R.S., Braun, D.C., Cameron, R., Duvall, T.L., Hanasoge, S.M., Jackiewicz, J., Roth, M., Stahn, T., Thompson, M.J., Zharkov, S.: 2009b, Space Sci. Rev., erratum, in press. doi:10.1007/s11214-010-9688-1.

Gokhale, M.H., Zwaan, C.: 1972, Solar Phys. 26, 52. doi:10.1007/BF00155105.

Gough, D.O.: 1984, Phil. Trans. Roy. Soc. Lond. Ser. A 313, 27.

Gurman, J.B., House, L.L.: 1981, Solar Phys. 71, 5. doi:10.1007/BF00153602.

Haber, D.A., Hindman, B.W., Toomre, J., Bogart, R.S., Larsen, R.M., Hill, F.: 2002, Astrophys. J. 570, 855. doi:10.1086/339631.

Haber, D.A., Hindman, B.W., Toomre, J., Thompson, M.J.: 2004, Solar Phys. 220, 371. doi:10.1023/B: SOLA.0000031405.52911.08.

Hagyard, M.J., West, E.A., Cumings, N.P.: 1977, Solar Phys. 53, 3. doi:10.1007/BF02260205.

Hanasoge, S.M.: 2008, Astrophys. J. 680, 1457. doi:10.1086/587934.

Hanasoge, S.M.: 2009, Astron. Astrophys. 503, 595. doi:10.1051/0004-6361/200912449.

Hanasoge, S.M.: 2010, Astron. Astrophys., in press.

Hanasoge, S.M., Duvall, T.L. Jr., Couvidat, S.: 2007, Astrophys. J. 664, 1234. doi:10.1086/519070.

Hanasoge, S.M., Komatitsch, D., Gizon, L.: 2010, Astron. Astrophys., in press. doi:10.1051/0004-6361/ 201014345.

Hanasoge, S.M., Duvall, T.L., DeRosa, M.L.: 2010, Astrophys. J. Lett. 712, L98. doi:10.1088/2041-8205/ 712/1/L98.

Hanasoge, S.M., Larsen, R.M., Duvall, T.L. Jr., DeRosa, M.L., Hurlburt, N.E., Schou, J., Roth, M., Christensen-Dalsgaard, J., Lele, S.K.: 2006, Astrophys. J. 648, 1268. doi:10.1086/505927.

Hanasoge, S.M., Couvidat, S., Rajaguru, S.P., Birch, A.C.: 2008, Mon. Not. Roy. Astron. Soc. 1291. doi:10.1111/j.1365-2966.2008.14013.x.

Hansen, S.C., Cally, P.S.: 2009, Solar Phys. 255, 193. doi:10.1007/s11207-009-9320-2.

Hartlep, T., Miesch, M.S., Mansour, N.N.: 2008, arXiv:0805.0333.

Harvey, K., Harvey, J.: 1973, Solar Phys. 28, 61. doi:10.1007/BF00152912.

Heinemann, T., Nordlund, Å., Scharmer, G.B., Spruit, H.C.: 2007, Astrophys. J. 669, 1390. doi:10.1086/520827.

Hindman, B.W., Brown, T.M.: 1998, Astrophys. J. 504, 1029. doi:10.1086/306128.

Hu, F.: 1996, J. Comput. Phys. 124, 177. doi:10.1006/jcph.1996.0052.

Hurlburt, N.E., Rucklidge, A.M.: 2000, Mon. Not. Roy. Astron. Soc. 314, 793. doi:10.1046/j.1365-8711. 2000.03407.x.

Ichimoto, K., Suematsu, Y., Tsuneta, S., Katsukawa, Y., Shimizu, T., Shine, R.A., Tarbell, T.D., Title, A.M., Lites, B.W., Kubo, M., Nagata, S.: 2007, Science 318, 1597. doi:10.1126/science.1146337.

Ichimoto, K., Suematsu, Y., Katsukawa, Y., Tsuneta, S., Shimojo, M., Shimizu, T., Shine, R.A., Tarbell, T.D., Berger, T., Title, A.M., Lites, B.W., Kubo, M., Yokoyama, T., Nagata, S.: 2009, In: Berdyugina, S.V., Nagendra, K.N., Ramelli, R. (eds.) CS-405, Astron. Soc. Pacific, San Francisco, 167.

Jackiewicz, J., Gizon, L., Birch, A.C.: 2008, Solar Phys. 251, 381. doi:10.1007/s11207-008-9158-z.

Jacoutot, L., Kosovichev, A.G., Wray, A., Mansour, N.N.: 2008, Astrophys. J. Lett. 684, L51. doi:10.1086/592042.

Jahn, K.: 1989, Astron. Astrophys. 222, 264.

Jahn, K.: 1992, In: Thomas, J.H., Weiss, N.O. (eds.) Sunspots. Theory and Observations, NATO ASIC Proc. 375, Kluwer, Dordrecht, 139. 
Jahn, K., Schmidt, H.U.: 1994, Astron. Astrophys. 290, 295.

Jakimiec, J.: 1965, Acta Astron. 15, 145.

Jakimiec, J., Zabża, M.: 1966, Acta Astron. 16, 73.

Jensen, J.M., Jacobsen, B.H., Christensen-Dalsgaard, J.: 1998, In: Korzennik, S. (ed.) Structure and Dynamics of the Interior of the Sun and Sun-like Stars SP-418, ESA, Noordwijk, 635.

Jurčák, J., Bellot Rubio, L.R.: 2008, Astron. Astrophys. 481, L17. doi:10.1051/0004-6361:20079021.

Kashyap, V.L., Drake, J.J., Saar, S.H.: 2008, Astrophys. J. 687, 1339. doi:10.1086/591922.

Keppens, R., Martínez Pillet, V.: 1996, Astron. Astrophys. 316, 229.

Khomenko, E., Collados, M.: 2006, Astrophys. J. 653, 739. doi:10.1086/507760.

Khomenko, E., Collados, M.: 2008, Astrophys. J. 689, 1379. doi:10.1086/592681.

Khomenko, E., Collados, M.: 2009, Astron. Astrophys. 506, L5. doi:10.1051/0004-6361/200913030.

Khomenko, E., Collados, M., Felipe, T.: 2008, Solar Phys. 251, 589. doi:10.1007/s11207-008-9133-8.

Khomenko, E., Kosovichev, A., Collados, M., Parchevsky, K., Olshevsky, V.: 2009, Astrophys. J. 694, 411. doi:10.1088/0004-637X/694/1/411.

Kitai, R., Watanabe, H., Nakamura, T., Otsuji, K.I., Matsumoto, T., Ueno, S., Nagata, S., Shibata, K., Muller, R., Ichimoto, K., Tsuneta, S., Suematsu, Y., Katsukawa, Y., Shimizu, T., Tarbell, T.D., Shine, R.A., Title, A.M., Lites, B.: 2007, Pub. Astron. Soc. Japan 59, 585.

Kitchatinov, L.L., Rüdiger, G.: 2007, In: Kneer, F., Puschmann, K.G., Wittmann, A.D. (eds.) Modern Solar Facilities-Advanced Solar Science, Universitätsverlag Göttingen, Göttingen, 343.

Kjeldseth Moe, O., Maltby, P.: 1969, Solar Phys. 8, 275. doi:10.1007/BF00155375.

Kjeldseth Moe, O., Maltby, P.: 1974, Solar Phys. 36, 101.

Komm, R., Howe, R., Hill, F.: 2009, Solar Phys. 258, 13. doi:10.1007/s11207-009-9398-6.

Komm, R., Howe, R., Hill, F., González-Hernández, I., Toner, C., Corbard, T.: 2005, Astrophys. J. $631,636$. doi:10.1086/432413.

Komm, R., Howe, R., Hill, F., González Hernández, I.: 2009, Solar Phys. 254, 1. doi:10.1007/ s11207-008-9285-6.

Kopp, G., Rabin, D.: 1992, Solar Phys. 141, 253. doi:10.1007/BF00155178.

Kosovichev, A.G.: 1996, Astrophys. J. Lett. 461, L55. doi:10.1086/309989.

Kosovichev, A.G., Duvall, T.L. Jr., Scherrer, P.H.: 2000, Solar Phys. 192, 159.

Kuridze, D., Zaqarashvili, T.V., Shergelashvili, B.M., Poedts, S.: 2008, Ann. Geophys. 26, 2983.

Kuridze, D., Zaqarashvili, T.V., Shergelashvili, B.M., Poedts, S.: 2009, Astron. Astrophys. 505, 763. doi:10.1051/0004-6361/200811484.

Landman, D.A., Finn, G.D.: 1979, Solar Phys. 63, 221. doi:10.1007/BF00174528.

Langhans, K., Scharmer, G.B., Kiselman, D., Löfdahl, M.G., Berger, T.E.: 2005, Astron. Astrophys. 436, 1087. doi:10.1051/0004-6361:20052678.

Lanza, A.F.: 2008, Astron. Astrophys. 487, 1163. doi:10.1051/0004-6361:200809753.

Leighton, R.B.: 1969, Astrophys. J. 156, 1. doi:10.1086/149943.

Leka, K.D., Skumanich, A.: 1998, Astrophys. J. 507, 454. doi:10.1086/306297.

Lites, B.W., Skumanich, A.: 1982, Astrophys. J. Suppl. 49, 293. doi:10.1086/190800.

Lites, B.W., Skumanich, A.: 1990, Astrophys. J. 348, 747. doi:10.1086/168284.

Liu, Q.Z., Song, M.T.: 1996, Chin. Astron. Astrophys. 20, 291.

Livingston, W.: 2002, Solar Phys. 207, 41.

Livingston, W., Harvey, J.W., Malanushenko, O.V., Webster, L.: 2006, Solar Phys. $239,41$. doi:10.1007/s11207-006-0265-4.

Low, B.C.: 1975, Astrophys. J. 197, 251. doi:10.1086/153508.

Low, B.C.: 1980, Solar Phys. 67, 57. doi:10.1007/BF00146682.

Magara, T., Longcope, D.W.: 2001, Astrophys. J. Lett. 559, L55. doi:10.1086/323635.

Magara, T., Longcope, D.W.: 2003, Astrophys. J. 586, 630. doi:10.1086/367611.

Makita, M., Morimoto, M.: 1960, Pub. Astron. Soc. Japan 12, 63.

Maltby, P.: 1964, Astrophys. Norv. 8, 205.

Maltby, P., Avrett, E.H., Carlsson, M., Kjeldseth-Moe, O., Kurucz, R.L., Loeser, R.: 1986, Astrophys. J. 306, 284. doi:10.1086/164342.

Mamadazimov, M.: 1972, Solar Phys. 22, 129. doi:10.1007/BF00145467.

Manchester, W. IV, Gombosi, T., DeZeeuw, D., Fan, Y.: 2004, Astrophys. J. 610, 588. doi:10.1086/421516.

Martens, P.C.H., Hurlburt, N.E., Title, A.M., Acton, L.W.: 1996, Astrophys. J. 463, 372. doi:10.1086/177251.

Martínez Pillet, V.: 1997, In: Schmieder, B., del Toro Iniesta, J.C., Vazquez, M. (eds.) 1st Advances in Solar Physics Euroconference, Advances in Physics of Sunspots CS-118, Astron. Soc. Pacific, San Francisco, 212.

Martínez Pillet, V.: 2000, Astron. Astrophys. 361, 734.

Martínez Pillet, V.: 2002, Astron. Nachr. 323, 342. doi:10.1002/1521-3994(200208)323:3/4.

Martínez Pillet, V., Vazquez, M.: 1993, Astron. Astrophys. 270, 494. 
Mason, D., Komm, R., Hill, F., Howe, R., Haber, D., Hindman, B.W.: 2006, Astrophys. J. $645,1543$. doi:10.1086/503761.

Mathew, S.K., Lagg, A., Solanki, S.K., Collados, M., Borrero, J.M., Berdyugina, S., Krupp, N., Woch, J., Frutiger, C.: 2003, Astron. Astrophys. 410, 695. doi:10.1051/0004-6361:20031282.

Mathew, S.K., Solanki, S.K., Lagg, A., Collados, M., Borrero, J.M., Berdyugina, S.: 2004, Astron. Astrophys. 422, 693. doi:10.1051/0004-6361:20040136.

Mathew, S.K., Martínez Pillet, V., Solanki, S.K., Krivova, N.A.: 2007, Astron. Astrophys. 465, 291. doi:10.1051/0004-6361:20066356.

Mazzucconi, F., Coveri, C., Godoli, G.: 1990, Solar Phys. 125, 269. doi:10.1007/BF00158406.

McIntosh, P.S.: 1981, In: Cram, L.E., Thomas, J.H. (eds.) The Physics of Sunspots, Sacramento Peak Observatory, Sunspot, 7.

Meyer, F., Schmidt, H.U.: 1968, Mitt. Astron. Ges. Hamb. 25, 194.

Montesinos, B., Thomas, J.H.: 1997, Nature 390, 485. doi:10.1038/37307.

Moon, Y.J., Yun, H.S., Park, J.S.: 1998, Astrophys. J. 494, 851. doi:10.1086/305239.

Moradi, H., Cally, P.S.: 2008, Solar Phys. 251, 309. doi:10.1007/s11207-008-9190-z.

Moradi, H., Hanasoge, S.M.: 2010, In: Hasan, S.S., Rutten, R.J. (eds.) Magnetic Coupling between the Interior and Atmosphere of the Sun, Astrophys. Space Sci. Proc., Springer, Berlin, 378. doi:10.1007/978-3-642-02859-5_35.

Moradi, H., Hanasoge, S.M., Cally, P.S.: 2009, Astrophys. J. Lett. 690, L72. doi:10.1088/0004-637X/ 690/1/L72.

Moretti, P.F., Jefferies, S.M., Armstrong, J.D., McIntosh, S.W.: 2007, Astron. Astrophys. 471, 961. doi:10.1051/0004-6361:20077247.

Nicolas, K.R., Bartoe, J.D.F., Brueckner, G.E., Kjeldseth-Moe, D.: 1981, In: Cram, L.E., Thomas, J.H. (eds.) The Physics of Sunspots, Sacramento Peak Observatory, Sunspot, 167.

Nordlund, Å., Scharmer, G.B.: 2010, In: Hasan, S.S., Rutten, R.J. (eds.) Magnetic Coupling between the Interior and Atmosphere of the Sun, Astrophys. Space Sci. Proc., Springer, Berlin, 243. doi:10.1007/978-3-642-02859-5_18.

Norton, A.A., Gilman, P.A.: 2004, Astrophys. J. 603, 348. doi:10.1086/381362.

Osherovich, V., Flaa, T.: 1983, Solar Phys. 88, 109. doi:10.1007/BF00196181.

Osherovich, V.A.: 1982, Solar Phys. 77, 63. doi:10.1007/BF00156095.

Osherovich, V.A., Garcia, H.A.: 1989, Astrophys. J. 336, 468. doi:10.1086/167025.

Osherovich, V.A., Lawrence, J.K.: 1983, Solar Phys. 88, 117. doi:10.1007/BF00196182.

Parchevsky, K.V., Kosovichev, A.G.: 2007, Astrophys. J. 666, 547. doi:10.1086/520108.

Parchevsky, K.V., Kosovichev, A.G.: 2009, Astrophys. J. 694, 573. doi:10.1088/0004-637X/694/1/573.

Parker, E.N.: 1975, Solar Phys. 40, 291. doi:10.1007/BF00162376.

Parker, E.N.: 1979, Astrophys. J. 234, 333. doi:10.1086/157501.

Penn, M.J., Livingston, W.: 2006, Astrophys. J. Lett. 649, L45. doi:10.1086/508345.

Petrovay, K., Moreno-Insertis, F.: 1997, Astrophys. J. 485, 398. doi:10.1086/304404.

Pizzo, V.J.: 1986, Astrophys. J. 302, 785. doi:10.1086/164041.

Pizzo, V.J.: 1990, Astrophys. J. 365, 764. doi:10.1086/169530.

Priest, E., Forbes, T.: 2000, Magnetic Reconnection, Cambridge University Press, Cambridge.

Puschmann, K.G., Ruiz Cobo, B., Martínez Pillet, V.: 2008, arXiv:0810.2432.

Rabello-Soares, M.C., Basu, S., Christensen-Dalsgaard, J.: 1999, Mon. Not. Roy. Astron. Soc. 309, 35. doi:10.1046/j.1365-8711.1999.02785.x.

Rajaguru, S.P., Birch, A.C., Duvall, T.L. Jr., Thompson, M.J., Zhao, J.: 2006, Astrophys. J. 646, 543. doi:10.1086/504705.

Rajaguru, S.P., Sankarasubramanian, K., Wachter, R., Scherrer, P.H.: 2007, Astrophys. J. Lett. 654, L175. doi:10.1086/511266.

Rast, M.P., Fox, P.A., Lin, H., Lites, B.W., Meisner, R.W., White, O.R.: 1999, Nature 401, 678. doi: $10.1038 / 44343$.

Rast, M.P., Meisner, R.W., Lites, B.W., Fox, P.A., White, O.R.: 2001, Astrophys. J. 557, 864. doi:10.1086/321673.

Rempel, M., Schüssler, M., Knölker, M.: 2009, Astrophys. J. 691, 640. doi:10.1088/0004-637X/691/1/640.

Rempel, M., Schüssler, M., Cameron, R.H., Knölker, M.: 2009, Science 325, 171. doi:10.1126/science.1173798.

Riethmüller, T.L., Solanki, S.K., Zakharov, V., Gandorfer, A.: 2008, Astron. Astrophys. 492, 233. doi:10.1051/0004-6361:200810701.

Rimmele, T.: 2008, Astrophys. J. 672, 684. doi:10.1086/523702.

Rimmele, T.R.: 1997, Astrophys. J. 490, 458. doi:10.1086/304863.

Ringnes, T.S., Jensen, E.: 1960, Astrophys. Norv. 7, 99.

Roden, A.J., Gedney, S.D.: 2000, Microwave Opt. Tech. Lett. 27, 334. 
Rossbach, M., Schröter, E.H.: 1970, Solar Phys. 12, 95. doi:10.1007/BF02276568.

Rouppe van der Voort, L.H.M.: 2002, Astron. Astrophys. 389, 1020. doi:10.1051/0004-6361:20020638.

Rucklidge, A.M., Schmidt, H.U., Weiss, N.O.: 1995, Mon. Not. Roy. Astron. Soc. 273, 491.

Ruiz Cobo, B., del Toro Iniesta, J.C.: 1992, Astrophys. J. 398, 375. doi:10.1086/171862.

Sainz Dalda, A., Bellot Rubio, L.R.: 2008a, Astron. Astrophys. 481, L21. doi:10.1051/0004-6361:20079115.

Sainz Dalda, A., Bellot Rubio, L.R.: 2008b, Astron. Astrophys. 481, L21. doi:10.1051/0004-6361:20079115.

Scharmer, G.B.: 2009, Space Sci. Rev. 144, 229. doi:10.1007/s11214-008-9483-4.

Scharmer, G.B., Spruit, H.C.: 2006, Astron. Astrophys. 460, 605. doi:10.1051/0004-6361:20066019.

Scharmer, G.B., Nordlund, Å., Heinemann, T.: 2008, Astrophys. J. Lett. 677, L149. doi:10.1086/587982.

Scharmer, G.B., Gudiksen, B.V., Kiselman, D., Löfdahl, M.G., Rouppe van der Voort, L.H.M.: 2002, Nature 420, 151.

Schlichenmaier, R.: 2002, Astron. Nachr. 323, 303. doi:10.1002/1521-3994(200208)323:3/4.

Schlichenmaier, R.: 2009, Space Sci. Rev. 144, 213. doi:10.1007/s11214-008-9465-6.

Schlichenmaier, R., Collados, M.: 2002, Astron. Astrophys. 381, 668. doi:10.1051/0004-6361:20011459.

Schlichenmaier, R., Schmidt, W.: 2000, Astron. Astrophys. 358, 1122.

Schlichenmaier, R., Bellot Rubio, L.R., Tritschler, A.: 2004, Astron. Astrophys. 415, 731. doi:10.1051/0004-6361:20034430.

Schlichenmaier, R., Jahn, K., Schmidt, H.U.: 1998, Astron. Astrophys. 337, 897.

Schlüter, A., Temesváry, S.: 1958, In: Lehnert, B. (ed.) Electromagnetic Phenomena in Cosmical Physics, IAU Sympos. 6, Cambridge University Press, Cambridge, 263.

Schmidt, H.U., Wegmann, R.: 1983, In: Brosowski, B., Martensen, E. (eds.) Dynamical Problems in Mathematical Physics, Peter Lang, Bern, 137.

Schmidt, W., Balthasar, H.: 1994, Astron. Astrophys. 283, 241.

Schmidt, W., Hofmann, A., Balthasar, H., Tarbell, T.D., Frank, Z.A.: 1992, Astron. Astrophys. 264, L27.

Schrijver, C.J.: 2001, Astrophys. J. 547, 475. doi:10.1086/318333.

Schrijver, C.J., Title, A.M.: 1999, Solar Phys. 188, 331.

Schunker, H., Braun, D.C. 2010, Solar Phys., doi:10.1007/s11207-010-9550-3.

Schunker, H., Cally, P.S.: 2006, Mon. Not. Roy. Astron. Soc. 372, 551. doi:10.1111/j.1365-2966. 2006.10855.x.

Schunker, H., Cameron, R., Gizon, L.: 2010, In: Elsworth, Y., Thompson, M. (eds.) Proceedings of the HELAS Workshop NA3-4 “The Acoustic Solar Cycle”, Univ. Birmingham, 6-8 January 2009. arXiv:1002.1969.

Schüssler, M.: 1984, Astron. Astrophys. 140, 453.

Schüssler, M.: 1987, In: Durney, B.R., Sofia, S. (eds.) The Internal Solar Angular Velocity, Astrophys. Space Sci. Lib. 137, Reidel, Dordrecht, 303.

Schüssler, M., Rempel, M.: 2005, Astron. Astrophys. 441, 337. doi:10.1051/0004-6361:20052962.

Schüssler, M., Vögler, A.: 2006, Astrophys. J. Lett. 641, L73. doi:10.1086/503772.

Schüssler, M., Caligari, P., Ferriz-Mas, A., Moreno-Insertis, F.: 1994, Astron. Astrophys. 281, L69.

Severino, G., Gomez, M.T., Caccin, B.: 1994, In: Rutten, R.J., Schrijver, C.J. (eds.) Solar Surface Magnetism, Kluwer, Dordrecht, 169.

Sheeley, N.R. Jr.: 1969, Solar Phys. 9, 347. doi:10.1007/BF02391657.

Sheeley, N.R. Jr.: 1972, Solar Phys. 25, 98. doi:10.1007/BF00155747.

Shelyag, S., Fedun, V., Erdélyi, R.: 2008, Astron. Astrophys. 486, 655. doi:10.1051/0004-6361:200809800.

Shelyag, S., Zharkov, S., Fedun, V., Erdélyi, R., Thompson, M.J.: 2009, Astron. Astrophys. 501, 735. doi:10.1051/0004-6361/200911709.

Shkolnik, E., Walker, G.A.H., Bohlender, D.A.: 2003, Astrophys. J. 597, 1092. doi:10.1086/378583.

Simon, G.W., Weiss, N.O.: 1970, Solar Phys. 13, 85. doi:10.1007/BF00963944.

Simon, G.W., Weiss, N.O., Nye, A.H.: 1983, Solar Phys. 87, 65. doi:10.1007/BF00151161.

Skumanich, A., Lites, B.W., Martínez Pillet, V.: 1994, In: Rutten, R.J., Schrijver, C.J. (eds.) Solar Surface Magnetism, Kluwer, Dordrecht, 99.

Sobotka, M.: 1997, In: Schmieder, B., del Toro Iniesta, J.C., Vazquez, M. (eds.) 1st Advances in Solar Physics Euroconference. Advances in Physics of Sunspots CS-118, Astron. Soc. Pacific, San Francisco, 155.

Sobotka, M.: 2002, In: Wilson, A. (ed.) Solar Variability: From Core to Outer Frontiers SP-506, ESA, Noordwijk, 381.

Sobotka, M., Bonet, J.A., Vazquez, M.: 1993, Astrophys. J. 415, 832. doi:10.1086/173205.

Socas-Navarro, H.: 2002, In: Sawaya-Lacoste, H. (ed.) SOLMAG 2002. Proceedings of the Magnetic Coupling of the Solar Atmosphere Euroconference SP-505, ESA, Noordwijk, 45.

Socas-Navarro, H.: 2005, Astrophys. J. Lett. 631, L167. doi:10.1086/497334.

Socas-Navarro, H.: 2007, Astrophys. J. Suppl. 169, 439. doi:10.1086/510336.

Socas-Navarro, H., Pillet, V.M., Sobotka, M., Vázquez, M.: 2004, Astrophys. J. 614, 448. doi:10.1086/ 423487. 
Solanki, S.K.: 1990, In: Stenflo, J.O. (ed.) Solar Photosphere: Structure, Convection, and Magnetic Fields, IAU Sympos. 138, Kluwer, Dordrecht, 103.

Solanki, S.K.: 2003, Astron. Astrophys. Rev. 11, 153. doi:10.1007/s00159-003-0018-4.

Solanki, S.K., Montavon, C.A.P.: 1993, Astron. Astrophys. 275, 283.

Solanki, S.K., Schmidt, H.U.: 1993, Astron. Astrophys. 267, 287.

Solanki, S.K., Unruh, Y.C.: 2004, Mon. Not. Roy. Astron. Soc. 348, 307. doi:10.1111/j.1365-2966. 2004.07368.x.

Solanki, S.K., Walther, U., Livingston, W.: 1993, Astron. Astrophys. 277, 639.

Solov'ev, A.A.: 1997, Astron. Rep. 41, 121.

Spruit, H.C.: 1976, Solar Phys. 50, 269. doi:10.1007/BF00155292.

Spruit, H.C.: 1981, In: Cram, L.E., Thomas, J.H. (eds.) The Physics of Sunspots, Sacramento Peak Observatory, Sunspot, 98.

Spruit, H.C.: 2003, Solar Phys. 213, 1.

Spruit, H.C., Roberts, B.: 1983, Nature 304, 401. doi:10.1038/304401a0.

Spruit, H.C., Scharmer, G.B.: 2006, Astron. Astrophys. 447, 343. doi:10.1051/0004-6361:20054000.

Staude, J.: 1986, Contrib. Astron. Obs. Skaln. Pleso 15, 299.

Staude, J., Hildebrandt, J., Fuerstenberg, F., Krueger, A., Jakimiec, J., Obridko, V.N., Siarkowski, M., Sylwester, B., Sylwester, J.: 1983, Acta Astron. 33, 441.

Stein, R.F., Nordlund, A.: 1998, Astrophys. J. 499, 914. doi:10.1086/305678.

Steiner, O., Pneuman, G.W., Stenflo, J.O.: 1986, Astron. Astrophys. 170, 126.

Steiner, O., Rezaei, R., Schaffenberger, W., Wedemeyer-Böhm, S.: 2008, Astrophys. J. Lett. 680, L85. doi:10.1086/589740.

Steiner, O., Rezaei, R., Schlichenmaier, R., Schaffenberger, W., Wedemeyer-Böhm, S.: 2009, In: Lites, B., Cheung, M., Magara, T., Mariska, J., Reeves, K. (eds.) CS-415, Astron. Soc. Pacific, San Francisco, 67.

Stellmacher, G., Wiehr, E.: 1975, Astron. Astrophys. 45, 69.

Strassmeier, K.G.: 1999, Astron. Astrophys. 347, 225.

Strassmeier, K.G.: 2009, Astron. Astrophys. Rev., 6. doi:10.1007/s00159-009-0020-6.

Strous, L.H., Scharmer, G., Tarbell, T.D., Title, A.M., Zwaan, C.: 1996, Astron. Astrophys. 306, 947.

Svanda, M., Klvaňa, M., Sobotka, M.: 2009, Astron. Astrophys. 506, 875. doi:10.1051/0004-6361/ 200912422.

Teplitskaya, R.B., Grigor'eva, S.A., Skochilov, V.G.: 1977, Issled. Geomagn. Aeron. Fiz. Soln. $42,48$.

Thomas, J.H., Weiss, N.: 1992, Sunspots. Theory and Observations, NATO ASIC Proc. 375, Kluwer, Dordrecht.

Thomas, J.H., Weiss, N..: 2008, Sunspots and Starspots, Cambridge University Press, Cambridge.

Thomas, J.H., Weiss, N.O.: 2004, Ann. Rev. Astron. Astrophys. 42, 517.

Thomas, J.H., Weiss, N.O., Tobias, S.M., Brummell, N.H.: 2002, Nature 420, 390.

Thompson, M.J., Zharkov, S.: 2008, Solar Phys. 251, 225. doi:10.1007/s11207-008-9143-6.

Tildesley, M.J., Weiss, N.O.: 2004, Mon. Not. Roy. Astron. Soc. 350, 657. doi:10.1111/ j.1365-2966.2004.07678.x.

Title, A.M., Frank, Z.A., Shine, R.A., Tarbell, T.D., Topka, K.P., Scharmer, G., Schmidt, W.: 1992, In: Thomas, J.H., Weiss, N.O. (eds.) Sunspots. Theory and Observations, NATO ASIC Proc. 139, Kluwer, Dordrecht, 195.

Title, A.M., Frank, Z.A., Shine, R.A., Tarbell, T.D., Topka, K.P., Scharmer, G., Schmidt, W.: 1993, Astrophys. J. 403, 780. doi:10.1086/172249.

Tobias, S., Weiss, N.: 2004, Astron. Geophys. 45(4), 040000. doi:10.1046/j.1468-4004.2003.45428.x.

Toth, G., Keppens, R., Botchev, M.A.: 1998, Astron. Astrophys. 332, 1159.

van Ballegooijen, A.A., Cartledge, N.P., Priest, E.R.: 1998, Astrophys. J. 501, 866. doi:10.1086/305823.

Vargas Domínguez, S., Bonet, J.A., Martínez Pillet, V., Katsukawa, Y., Kitakoshi, Y., Rouppe van der Voort, L.: 2007, Astrophys. J. Lett. 660, L165. doi:10.1086/518123.

Vernazza, J.E., Avrett, E.H., Loeser, R.: 1981, Astrophys. J. Suppl. 45, 635. doi:10.1086/190731.

Vrabec, D.: 1971, In: Howard, R. (ed.) Solar Magnetic Fields, IAU Sympos. 43, 329.

Vrabec, D.: 1974, In: Athay, R.G. (ed.) Chromospheric Fine Structure, IAU Sympos. 56, Reidel, Dordrecht, 201.

Wang, H., Zirin, H.: 1992, Solar Phys. 140, 41. doi:10.1007/BF00148428.

Wang, Y.M., Nash, A.G., Sheeley, N.R. Jr.: 1989, Astrophys. J. 347, 529. doi:10.1086/168143.

Watson, F., Fletcher, L., Dalla, S., Marshall, S.: 2009, Solar Phys. doi:10.1007/s11207-009-9420-z.

Wegmann, R.: 1981, In: Albrecht, J., Collatz, L., Hoffmann, K.H. (eds.) Numerical Treatment of Free Boundary Value Problems, Birkhäuser, Stuttgart, 335.

Weinberg, S.: 1962, Phys. Rev. 126, 1899. doi:10.1103/PhysRev.126.1899.

Weiss, N.O.: 1966, Phil. Trans. Roy. Soc. Lond. Ser. A 293, 310. 
Weiss, N.O., Thomas, J.H., Brummell, N.H., Tobias, S.M.: 2004, Astrophys. J. 600, 1073. doi:10.1086/380091.

Westendorp Plaza, C., del Toro Iniesta, J.C., Ruiz Cobo, B., Pillet, V.M.: 2001, Astrophys. J. 547, 1148. doi:10.1086/318377.

Yang, G., Xu, Y., Wang, H., Denker, C.: 2003, Astrophys. J. 597, 1190. doi:10.1086/377128.

Yun, H.S.: 1970, Astrophys. J. 162, 975. doi:10.1086/150729.

Yun, H.S.: 1971, Solar Phys. 16, 398. doi:10.1007/BF00162481.

Yun, H.S., Beebe, H.A., Baggett, W.E.: 1984, Solar Phys. 92, 145. doi:10.1007/BF00157241.

Yurchyshyn, V.B., Wang, H., Goode, P.R.: 2001, Astrophys. J. 550, 470. doi:10.1086/319717.

Zel'dovich, Y.B.: 1956, Sov. J. Exp. Theor. Phys. 31, 154.

Zhao, J., Kosovichev, A.G.: 2003, Astrophys. J. 591, 446.

Zhao, J., Kosovichev, A.G.: 2004, Astrophys. J. 603, 776. doi:10.1086/381489.

Zhao, J., Kosovichev, A.G., Duvall, T.L. Jr.: 2004, Astrophys. J. Lett. 607, L135. doi:10.1086/421974.

Zwaan, C.: 1978, Solar Phys. 60, 213. doi:10.1007/BF00156523.

Zwaan, C.: 1981, Space Sci. Rev. 28, 385. doi:10.1007/BF00212600.

Zwaan, C.: 1992, In: Thomas, J.H., Weiss, N.O. (eds.) Sunspots. Theory and Observations, NATO ASIC Proc. 375, Kluwer, Dordrecht, 75. 